\title{
AXIOMATIC AND ECONOMIC APPROACHES TO INTERNATIONAL COMPARISONS
}

W. Erwin Diewert

Working Paper 5559

\author{
NATIONAL BUREAU OF ECONOMIC RESEARCH \\ 1050 Massachusetts Avenue \\ Cambridge, MA 02138 \\ May 1996
}

This research was supported by a SSHRC grant. The author thanks Bert Balk and Alice Nakamura for helpful suggestions and Tina Corsi for typing a difficult manuscript. This paper is part of NBER's research programs in International Trade and Investment, and Productivity. Any opinions expressed are those of the author and not those of the National Bureau of Economic Research.

(C) 1996 by W. Erwin Diewert. All rights reserved. Short sections of text, not to exceed two paragraphs, may be quoted without explicit permission provided that full credit, including (C) notice, is given to the source. 


\title{
AXIOMATIC AND ECONOMIC APPROACHES TO INTERNATIONAL COMPARISONS
}

\begin{abstract}
The paper considers the problem of choosing a multilateral system of index numbers in order to make aggregate price and quantity comparisons between many countries and regions. The problems involved in choosing functional forms in order to make these comparisons are both theoretically and empirically more difficult than the problems involved in choosing a bilateral index number formula. The great variation in relative prices and quantities in the international context means that the choice of a multilateral method is empirically important: different methods will give very different answers. The paper reviews ten classes of multilateral methods both from the viewpoint of the axiomatic approach as well as the economic approach. A new system of 11 desirable axioms or properties for multilateral systems is suggested. With respect to the economic approach, the concept of a superlative bilateral index number formula (which can adequately model substitution effects) is adapted to the multilateral context. Each of the 10 classes of multilateral methods is evaluated from the axiomatic and economic perspectives. Four classes of methods are identified as being "best". They are all superlative and satisfy slightly different sets of axioms.
\end{abstract}

W. Erwin Diewert Department of Economics University of British Columbia 997-1873 East Mall Vancouver, BC V6T 1Z1 CANADA and NBER 


\section{Introduction}

For a variety of reasons, it is useful to be able to make accurate comparisons of the relative consumption or real output between countries or between regions within a country; e.g., aid flows or interregional transfer payments may depend on these multilateral comparisons. Normal bilateral index number theory cannot be applied in this multilateral context because bilateral comparisons are inherently dependent on the choice of a base country and the resulting rankings of countries are not invariant to the choice of the base country. Moreover, it is usually politically unacceptable to have a single country or region play an asymmetric role in making multilateral comparisons.

The problem of making bilateral index number comparisons has been intensively studied for about a century. From the viewpoints of both the economic and test approaches to bilateral index number theory, a consensus has emerged that the Fisher [1922] ideal price and quantity indexes are probably the best functional forms for index number formulae. ${ }^{1}$ However, there is no comparable consensus on what is the appropriate method for making symmetric multilateral index number comparisons; i.e., comparisons that do not depend on the asymmetric choice of a base country. Part of the reason for this lack of consensus is that the test or axiomatic approach to multilateral index number theory is not as well developed as the bilateral theory. In the last decade, Diewert [1986] [1988], Balk [1989] [1995b] and Armstrong [1995] have made a start on developing axiomatic approaches to multilateral comparisons. ${ }^{2}$ In section 2 below, the present paper draws on this literature by suggesting a list of 12 desirable properties or tests for multilateral methods. In sections 4 to 13 , we evaluate 10 different multilateral methods from the perspective of this test approach to multilateral comparisons. We find that none of these methods satisfies all of our suggested tests. Thus it is necessary to make choices about the relative importance of the various tests.

In section 3 below, we suggest a multilateral generalization of the economic approach to making bilateral comparisons. In analogy to the bilateral case ${ }^{3}$, we say that a multilateral system is superlative if it is exact for a flexible linearly homogeneous aggregator function. In sections 4 to 13 , we determine whether the 10 multilateral methods studied in this paper are also superlative.

Section 14 discusses some of the tradeoffs between the various methods and section 15 concludes.

Appendix 1 contains proofs of various Propositions and Appendix 2 tables numerical results for the 10 multilateral methods for a 3 country, 2 commodity artifical data set. 


\section{Multilateral Axioms or Tests}

Suppose that the outputs, inputs or real consumption expenditures of $K$ countries ${ }^{4}$ in a bloc of countries are to be compared. Suppose also that there are $N$ homogeneous commodities consumed (or produced) in the $K$ countries during the time periods under consideration and the price and quantity of commodity $n$ in country $k$ are $p_{n}^{k}>0$ and $y_{n}^{k} \geq 0$ respectively for $n=1, \ldots, N$ and $k=1, \ldots, K .^{5}$ Denote the country $k$ price and quantity vector by $p^{k} \equiv\left[p_{1}^{k}, \ldots, p_{N}^{k}\right]^{T}>>0_{N}$ and $y^{k} \equiv\left[y_{1}^{k}, \ldots, y_{N}^{k}\right]^{T}>0_{N}$ respectively. $^{6}$ We assume that all prices are positive and are measured in common units and a common numeraire currency. We also assume that the aggregate bloc quantity vector is strictly positive; i.e., $\Sigma_{k=1}^{K} y^{k}>>0_{N}$. Finally, denote the $N$ by $K$ matrix of country prices by $P \equiv\left[p^{1}, \ldots, p^{K}\right]$ and the $N$ by $K$ matrix of country quantities by $Y \equiv\left[y^{1}, \ldots, y^{K}\right]$.

The share of bloc consumption (or output or input) for country $k, S^{k}$, will depend in general on the matrix of prices $P$ and the matrix of quantities $Y$. Thus $S^{k}$ will be a function of the components of $P$ and $Y$, say $S^{k}(P, Y)$ for $k=1, \ldots, K$. We assume that the domain of definition for these functions is the set of strictly positive country price vectors $p^{k}>>0_{N}$ and nonnegative but nonzero quantity vectors $y^{k}>0_{N}$ for $k=1, \ldots, K$ with $\Sigma_{k=1}^{K} y^{k}>>0_{N}$. In the remainder of this paper, we shall call a specific set of functions defined on the above domain of definition, $\left\{S^{1}(P, Y), \ldots, S^{K}(P, Y)\right\}$, a multilateral system of bloc share functions or a multilateral method for making international comparisons of aggregate quantities.

If there are only two units being compared, then define $Q\left(p^{1}, p^{2}, y^{1}, y^{2}\right) \equiv S^{2}\left(p^{1}\right.$, $\left.p^{2}, y^{1}, y^{2}\right) / S^{1}\left(p^{1}, p^{2}, y^{1}, y^{2}\right)$ as the ratio of "country" 2's share of "output" to "country" 1's share. The resulting function $Q\left(p^{1}, p^{2}, y^{1}, y^{2}\right)$ can be interpreted as a bilateral quantity index. We view a multilateral system as a generalization of bilateral index number theory to cover the situation where the number of units being compared is greater than two. In the remainder of this paper, we assume that the number of countries in the bloc is $K \geq 3$, (unless we explicitly assume $K=2$ ). If there is only one commodity, then there is no index number problem; i.e., we will have $S^{k}(P, Y)=y_{1}^{k} / \Sigma_{j=1}^{K} y_{1}^{j}$ for $k=1, \ldots, K$. Thus we also assume that the number of commodities is $N \geq 2$.

In the axiomatic approach to bilateral index number theory ${ }^{7}$, the function $Q\left(p^{1}, p^{2}\right.$, $\left.y^{1}, y^{2}\right)$ is hypothesized to satisfy various axioms or tests. We shall follow an analogous approach to multilateral index number theory by placing various tests or axioms on the multilateral system of share functions $S^{k}(P, Y)$.

Before we list our multilateral axioms, consider the following example of a multilateral system:

$$
S^{k}(P, Y) \equiv p^{k} \cdot y^{k} / \Sigma_{j=1}^{K} p^{j} \cdot y^{j} ; \quad k=1, \ldots, K
$$


The above system of multilateral share functions is the exchange rate system, where country $k$ 's share of bloc consumption (or output or input) is simply its share of total bloc value where all values are computed using a common numeraire currency.

We now list 12 desirable properties for multilateral systems. We regard the first 7 properties as being more essential.

Our first multilateral axiom is:

T1: Share Test: $\quad$ There exist $K$ continuous, positive functions $S^{k}(P, Y), k=1, \ldots, K$, such that $\Sigma_{k=1}^{K} S^{k}(P, Y)=1$ for all $P, Y$ in the domain of definition described above.

It is obvious that share functions must sum to unity. The above share test also added the requirements that each share function be continuous and positive. The test $T 1$ (without the positivity requirement) was proposed by Diewert $[1986 ; 36][1988 ; 76]$.

To motivate our second test, suppose that each country's share of bloc output is the same for every commodity, say $\beta_{k}$ for country $k$. Then it seems reasonable to ask that $S^{k}(P, Y)=\beta_{k}$ for each $k$.

T2: Proportional Quantities Test: Suppose $y^{k}=\beta_{k} y$ for $k=1, \ldots, K$ with $\beta_{k}>0$ and $\Sigma_{k=1}^{K} \beta_{k}=1$. Then $S^{k}(P, Y)=\beta_{k}$ for $k=1, \ldots, K$.

The above test is a multilateral counterpart to Leontief's [1936] Aggregation Theorem.

Our next test is a counterpart to Hicks' $[1946 ; 312-313]$ Aggregation Theorem: if each country's price vector $p^{k}$ is proportional to a common positive price vector $p$, then this $p$ can be used to determine country $k$ 's share of bloc output as $p \cdot y^{k} / \Sigma_{j=1}^{K} p \cdot y^{j}$.

T3: Proportional Prices Test: Suppose $p^{k}=\alpha_{k} p$ for $k=1, \ldots, K$ with $\alpha_{k}>0$ for some $p>>0_{N}$. Then $S^{k}(P, Y)=p \cdot y^{k} / p \cdot \Sigma_{j=1}^{K} y^{j}$ for $k=1, \ldots, K$.

Thus if either prices or quantities are proportional across countries, then tests T2 and T3 determine what the country share functions $S^{k}(P, Y)$ must be. The tests $T 2$ and $T 3$ can be interpreted as multilateral counterparts to identity tests for bilateral price and quantity indexes.

Our next 3 tests are invariance or symmetry tests.

T4: Commensurability Test (Invariance to Changes in the Units of Measurement): Let $\delta_{n}>0$ for $n=1, \ldots, N$ and let $\hat{\delta}$ denote the $N$ by $N$ diagonal matrix with the $\delta_{\mathfrak{n}}$ on the main diagonal. Then $S^{k}\left(\hat{\delta} P, \hat{\delta}^{-1} Y\right)=S^{k}(P, Y)$ for $k=1, \ldots, K$.

The test $T 4$ requires that the system of share functions be invariant to changes in the units of measurement for the $N$ commodities. In the multilateral context, this test was 
proposed by Diewert [1986; 38] [1988; 78]. In the bilateral context, this test was proposed by Jevons $[1884 ; 23]$, Pierson $[1896 ; 131]$ and Fisher $[1911 ; 411][1922 ; 420]$.

T5: Commodity Reversal Test (Invariance to the Ordering of Commodities): $\quad$ Let $\Pi$ denote an $N$ by $N$ permutation matrix. Then $S^{k}(\Pi P, \Pi Y)=S^{k}(P, Y)$ for $k=1, \ldots, K$.

The above test implies that a country's share of bloc output remains unchanged if the ordering of the $N$ commodities is unchanged. In the bilateral context, this test was first proposed by Fisher [1922; 63] and in the multilateral context by Diewert [1986; 39] [1988; 79].

T6: Multilateral Country Reversal Test (Symmetric Treatment of Countries): Let $S(P, Y)^{T} \equiv\left[S^{1}(P, Y), \ldots, S^{K}(P, Y)\right]$ denote the row vector of country share functions and let $\Pi^{*}$ denote a $K$ by $K$ permutation matrix. Then $S\left(P \Pi^{*}, Y \Pi^{*}\right)^{T}=S(P, Y)^{T} \Pi^{*}$.

Thus if the ordering of the countries is changed or permuted, then the resulting system of share functions is equal to the same permutation of the original share functions. The test T6 means that no country can play an asymmetric role in the definition of the country share functions. This property of a multilateral system was termed base country invariance by Kravis, Kenessey, Heston and Summers [1975]. When multilateral indexes are used by multinational agencies such as the European Union, the OECD or the World Bank, it is considered vital that the multilateral system satisfy $T 6$. This property can be viewed as a fairness test: each country must be treated in an even handed symmetric manner.

Our next test imposes the requirement that scale differences in the price levels of each country (or the use of different monetary units in each country) do not affect the country shares of bloc output.

T7: Monetary Units Test. Let $\alpha_{k}>0$ for $k=1, \ldots, K$. Then $S^{k}\left(\alpha_{1} p^{1}, \ldots, \alpha_{K} p^{K}, Y\right)$ $=S^{k}\left(p^{1}, \ldots, p^{K}, Y\right)$ for $k=1, \ldots, K$.

Mathematically, $T 7$ is a homogeneity of degree 0 in prices property, a property which is usually imposed on quantity indexes in bilateral index number theory. In the multilateral context, Gerardi [1982; 398] and Diewert [1986; 38] [1988; 78] proposed this test.

The test $T 7$ is a homogeneity in prices test. Our next test is a homogeneity in quantities test.

T8: Homogeneity in Quantities Test: For $i=1, \ldots, K, \lambda_{i}>0, j \neq i, j=1, \ldots, K$, we have $S^{i}\left(P, y^{1}, \ldots, y^{i-1}, \lambda_{i} y^{i}, y^{i+1}, \ldots, y^{K}\right) / S^{j}\left(P, y^{1}, \ldots, y^{i-1}, \lambda_{i} y^{i}, y^{i+1}, \ldots, y^{K}\right)=$ $\lambda_{i} S^{i}(P, Y) / S^{j}(P, Y)$. 
Mathematically, $T 8$ says that the output share of country $i$ relative to country $j, S^{i} / S^{j}$, is linearly homogeneous in the components of the country $i$ quantity vector $y^{i}$. This property is usually imposed on bilateral quantity indexes. In the multilateral context, this test was suggested by Gerardi $[1982 ; 397]$ and Diewert [1986; 37] [1988; 77].

Our next test imposes the following very reasonable property: as any component of country $k$ 's quantity vector $y^{k}$ increases, then country $k$ 's share of bloc output should also increase.

T9: Monotonicity Test: $S^{k}\left(P, y^{1}, \ldots, y^{k}, \ldots, y^{K}\right)$ is increasing in the components of the vector $y^{k}$ for $k=1, \ldots, K$ :

Although $T 9$ has not been proposed before in the multilateral context, it has been proposed in the context of bilateral index number theory; see Eichhorn and Voeller [1976; 23] and Vogt $[1980 ; 70]$.

The next two tests can be viewed as consistency in aggregation tests or country weighting tests.

T10: Country Partitioning Test: Let $A$ be a strict subset of the indices $\{1,2, \ldots, K\}$ with at least two members. Suppose $p^{i}=\alpha_{i} p^{a}$ for $\alpha_{i}>0, p^{a}>0_{N}$ and $y^{i}=\beta_{i} y^{a}$ for $\beta_{i}>0, y^{a} \gg 0_{N}$ for $i \in A$ with $\Sigma_{i \in A} \beta_{i}=1$. Denote the subset of $\{1,2, \ldots, K\}$ that does not belong to $A$ by $B$ and denote the matrices of country price and quantity vectors that do not belong to $A$ by $P^{b}$ and $Y^{b}$ respectively. Then: (i) for $i \in A, j \in$ $A, S^{i}(P, Y) / S^{j}(P, Y)=\beta_{i} / \beta_{j}$ and (ii) for $i \in B, S^{i}(P, Y)=S^{i *}\left(p^{a}, P^{b}, y^{a}, Y^{b}\right)$ where $S^{k *}\left(p^{a}, P^{b}, y^{a}, Y^{b}\right)$ is the system of share functions obtained by adding the bloc $A$ aggregate price and quantity vectors $p^{a}$ and $y^{a}$ to the bloc $B$ price and quantity matrices $P^{b}$ and $Y^{b}$.

Thus if the aggregate quantity vector for bloc $A, y^{a}$, were distributed proportionally among its bloc members and each bloc $A$ member's price vector were proportional to the price vector $p^{a}$, then part (i) of $T 10$ required that the bloc $A$ share functions reflect their proportional allocations of outputs and part (ii) of $T 10$ requires that the non bloc $A$ share functions yield the same numerical values if bloc $A$ were aggregated up into a single country (or conversely, the non bloc $A$ share functions yield the same values if a single bloc $A$ country is proportionally partitioned into smaller units). Note that $T 10$ requires that $K \geq 3$ and that the system of share functions be defined for varying numbers of countries. Test $T 10$ can be viewed as a generalization of Diewert's [1986; 40] [1988; 79] country partitioning test. For precursors of this type of test, see Hill [1982; 50] and Kravis, Summers and Heston [1982; 408]. Note that the countries in bloc $A$ satisfy the conditions for both Hicks and Leontief aggregation; i.e., both prices and quantities are proportional for bloc $A$ countries. Under these rather strong conditions, it seems very reasonable to ask 
that the system of share functions behave in the manner indicated by parts (i) and (ii) of T10.

The following test also uses combined Hicks and Leontief aggregation but it applies these aggregation conditions to countries in blocs $A$ and $B$.

T11: Bilateral Consistency in Aggregation Test: Let $A$ and $B$ be nonempty disjoint partitions of the country indices $\{1,2, \ldots, K\}$. Suppose that $p^{i}=\alpha_{i} p^{a}, y^{i}=\beta_{i} y^{a}, \alpha_{i}>$ $0, \beta_{i}>0, p^{a}>>0_{N}, y^{a}>>0_{N}$ for $i \in A$ with $\Sigma_{i \in A} \beta_{i}=1$ and $p^{j}=\gamma_{j} p^{b}, y^{j}=\delta_{j} y^{b}, \gamma_{j}>0$, $\delta_{j}>0, p^{b} \gg 0_{N}, y^{b}>0_{N}$ for $j \in B$ with $\Sigma_{j \in B} \delta_{j}=1$. Then $\Sigma_{j \in B} S^{j}(P, Y) / \Sigma_{i \in A}$ $S^{i}(P, Y)=Q_{F}\left(p^{a}, p^{b}, y^{a}, y^{b}\right)$ where $Q_{F}$ is the Fisher [1922] ideal quantity index defined by

$$
Q_{F}\left(p^{a}, p^{b}, y^{a}, y^{b}\right) \equiv\left[p^{a} \cdot y^{b} p^{b} \cdot y^{b} / p^{a} \cdot y^{a} p^{b} \cdot y^{a}\right]^{\frac{1}{2}}
$$

In this test, the set of countries is split up into two blocs of countries, $A$ and $B$. Within each bloc, price and quantity vectors are proportional. Hence if we aggregate country shares over blocs and divide the sum of the bloc $B$ shares by the sum of the bloc $A$ shares, we should get the same answer that the "best" bilateral index number formula $Q\left(p^{a}, p^{b}, y^{a}, y^{b}\right)$ would give, where the bloc $A$ and $B$ aggregate price and quantity vectors $p^{a}, p^{b}, y^{a}, y^{b}$ are used as arguments in the bilateral index number formula. We chose $Q$ to equal $Q_{F}$ since the Fisher ideal bilateral quantity index satisfies more "reasonable" bilateral tests than its competitors; see Diewert [1992; 214-223]. Of course, it is possible to modify test $T 11$ by replacing the Fisher ideal index $Q_{F}$ by an alternative "best" bilateral index number formula. However, the basic idea of test $T 11$ seems very reasonable: a good multilateral method should collapse down to a good bilateral method if all price and quantity vectors are proportional within blocs $A$ and $B$.

The test $T 11$ is related to Diewert's [1986; 41] [1988; 81] Strong Dependence on a Bilateral Formula Test. That test required that the limit of $S^{j}(P, y) / S^{i}(P, Y)$ equal a bilateral quantity index $Q\left(p^{i}, p^{j}, y^{i}, y^{j}\right)$ as all quantity vectors $y^{k}$ (except $y^{i}$ and $y^{j}$ ) tended to $0_{N}$. However, we regard the present Bilateral Consistency in Aggregation Test as a more satisfactory test, since some multilateral methods will not be well defined as quantity vectors tend to zero.

We regard all of the above tests as being very reasonable and desirable for a multilateral method. Unfortunately, none of the $\mathbf{1 0}$ multilateral methods that we study in this paper satisfy all of these tests.

Before we consider economic approaches to multilateral comparisons, we consider one additional test that practitioners regard as desirable.

We define an additive multilateral system of share functions $S^{k}(P, Y), k=1, \ldots, K$, as follows: there exist $N$ once continuously differentiable positive functions of $2 N K$ variables, $g_{n}(P, Y), n=1, \ldots, N$ such that 


$$
S^{k}(P, Y)=\Sigma_{n=1}^{N} g_{n}(P, Y) y_{n}^{k} / \Sigma_{m=1}^{N} g_{m}(P, Y) \Sigma_{j=1}^{K} y_{m}^{j} ; \quad k=1, \ldots, K
$$

where the functions $g_{n}$ have the following property:

$$
g_{n}(p, p, \ldots, p, Y)=p_{n} ; \quad n=1, \ldots, N
$$

for all $p>>0_{N}$ and $Y$ in the domain of definition where $p \equiv\left[p_{1}, \ldots, p_{N}\right]^{T}$ is a common price vector across all countries.

Property (3) is the main defining property of an additive system: it says that each country's share is determined by valuing its consumption components (or outputs or inputs) using the common "international" prices $g_{1}(P, Y), \ldots, g_{N}(P, Y)$, which in principle can depend on the entire matrices of country prices and quantities, $P \equiv\left[p^{1}, \ldots, p^{K}\right]$ and $Y \equiv\left[y^{1}, \ldots, y^{K}\right]$. Property (4) restricts the class of admissible "international" prices in a very sensible way: if all of the country prices are equal so that $p^{1}=p^{2}=\ldots=p^{K}=p$, then the "international" prices collapse down to these common national prices.

With the above definition in mind, we can state our last test:

T12: Additivity Test: the multilateral system is additive.

An additive multilateral system has the tremendously attractive feature of being user friendly: if an analyst wants to compare the relative performance of countries over subsets of commodities, then this can be done using the "international" prices $g_{n}(P, Y)$ to weight $y_{n}^{k}$ for each country $k$ and for $n$ belonging to the subset of commodities to be compared. There is no need to compute a separate set of country parities for each subset of commodities to be compared. Moreover, each commodity component will correctly aggregate up to bloc consumption (or output or input) valued at the international prices $g_{n}(P, Y)$.

Unfortunately, although additive multilateral methods are very convenient, they are not consistent with the economic approach to multilateral systems as we shall see.

We now turn to a description of an economic approach to making international comparisons.

\section{An Economic Approach To Multilateral Index Numbers}

The axiomatic approach to multilateral systems of index numbers does not make use of the assumption of optimizing behavior on the part of economic agents. Thus the country price and quantity vectors, $p^{k}$ and $y^{k}$, were treated as vectors of independent variables in the previous section. In this section, we follow the example of Diewert [1996; 19-25] and assume optimizing behavior on the part of economic agents in each country. 
Under this assumption, prices and quantities cannot be regarded as independent variables: given prices, quantities are determined (or vice versa).

We shall make the very strong assumption that a common linearly homogeneous aggregator function $f$ exists across countries. This is the assumption that was used by Diewert $[1976 ; 117]$ in his definition of a superlative bilateral index number formula. Thus in this section, we are looking for a multilateral counterpart to the bilateral concept of superlativeness. In the consumer context, ${ }^{8}$ we assume that each household in each country maximizes the increasing, concave and linearly homogeneous utility function $f(y)$ subject to its budget constraint. Aggregating over households in country $k$, we find that the country $k$ quantity vector $y^{k}$ is a solution to:

$$
\max _{y}\left\{f(y): p^{k} \cdot y=p^{k} \cdot y^{k}\right\} ; \quad k=1, \ldots, K
$$

Define the increasing, linearly homogeneous and concave unit cost function that is dual to $f$ by

$$
c(p) \equiv \min _{y}\{p \cdot y: f(y) \geq 1 ; y \geq 0,\}
$$

where $p>>0_{N}$ is a positive vector of commodity prices. If all consumers in country $k$ face the same prices $p^{k}$ and $y^{k}$ is the total consumption vector for country $k$, then we have (7) $\quad p^{k} \cdot y^{k}=c\left(p^{k}\right) f\left(y^{k}\right) ; \quad k=1, \ldots, K$.

Define the country $k$ aggregate utility level of $u_{k}$ and the country $k$ unit cost or unit expenditure $e_{k}$ as follows:

(8) $\quad u_{k} \equiv f\left(y^{k}\right) ; e_{k} \equiv c\left(p^{k}\right) ; \quad k=1, \ldots, K$

If the unit cost function $c$ is differentiable, then by Shephard's [1953; 11] Lemma, country $k$ quantities $y^{k}$ can be defined in terms of country $k$ prices $p^{k}$ and country $k$ aggregate utility $\boldsymbol{u}_{k}$ as follows:

(9) $\quad y^{k}=\nabla c\left(p^{k}\right) u_{k} ; \quad k=1, \ldots, K$

where $\nabla c\left(p^{k}\right) \equiv\left[c_{1}\left(p^{k}\right), \ldots, c_{N}\left(p^{k}\right)\right]^{T}$ is the vector of first order partial derivatives of $c$ evaluated at $p^{k}$.

On the other hand, if the utility function $f$ is differentiable, then by Wold's [1944; 69-71] Lemma, country $k$ prices $p^{k}$ can be defined in terms of country $k$ quantities $y^{k}$ and the country $k$ unit expenditure level $e_{k}$ as follows: ${ }^{9}$

$$
p^{k}=\nabla f\left(y^{k}\right) e_{k} ; \quad k=1, \ldots, K,
$$


where $\nabla f\left(y^{k}\right) \equiv\left[f_{1}\left(y^{k}\right), \ldots, f_{N}\left(y^{k}\right)\right]^{T}$ is the vector of first order partial derivatives of $f$ evaluated at $y^{k}$.

Under the above assumption of optimizing behavior on the part of economic agents for a linearly homogeneous aggregator function $f$, it is natural to ask that our system of multilateral share functions $S^{k}(P, Y)$ have the following exactness property:

$$
S^{i}(P, Y) / S^{j}(P, Y)=f\left(y^{i}\right) / f\left(y^{j}\right) ; \quad 1 \leq i, j \leq K
$$

Thus under the assumption of homogeneous utility maximization in all countries, it is natural to require that the ratio of the consumption shares for countries $i$ and $j, S^{i}(P, Y)$ $/ S^{j}(P, Y)$, be equal to the aggregate real consumption ratio for the two countries, $f\left(y^{i}\right)$ $/ f\left(y^{j}\right)$, for all countries $i$ and $j$.

The above preliminary definition of exactness (11) does not indicate whether we are regarding prices or quantities as independent variables. Thus, more precisely, we say that the multilateral system $S^{k}(P, Y), k=1, \ldots, K$ is exact for the differentiable homogeneous aggregator function $f^{10}$ if for all $y^{k}>0_{N}$ and $e_{k}>0$ for $k=1, \ldots, K$, we have

$$
\begin{aligned}
S^{i}\left[\nabla f\left(y^{1}\right) e_{1}, \ldots,\right. & \left.\nabla f\left(y^{K}\right) e_{K}, y^{1}, \ldots, y^{K}\right] / S^{j}\left[\nabla f\left(y^{1}\right) e_{1}, \ldots, \nabla f\left(y^{K}\right) e_{K}, y^{1}, \ldots, y^{K}\right] \\
& =f\left(y^{i}\right) / f\left(y^{j}\right) ; \quad 1 \leq i<j \leq K .
\end{aligned}
$$

In the above definition of exactness, we assume optimizing behavior, with prices $p^{k}$ in the share functions $S^{k}(P, Y)$ being replaced by the inverse demand functions $\nabla f\left(y^{k}\right) e_{k}$; see (10) above. Thus the weakly positive country quantity vectors $y^{k}>0_{N}$ and the positive country unit expenditure levels $e_{k}>0, k=1, \ldots, K$ are regarded as the independent variables in the system of functional equations defined by (12).

The above definition of exactness assumes that each country's system of inverse demand functions (10) exists. Turning now to the dual case where we assume that each country's system of Hicksian demand functions (9) exists, we say that the multilateral system $S^{k}(P, Y), k=1, \ldots, K$, is exact for the differentiable unit cost function $c^{11}$ if for all $p^{k}>>0_{N}$ and $u_{k}>0$ for $k=1, \ldots, K$, we have

$$
\begin{gathered}
S^{i}\left[p^{1}, \ldots, p^{K}, \nabla c\left(p^{1}\right) u_{1}, \ldots, \nabla c\left(p^{K}\right) u_{K}\right] / S^{j}\left[p^{1}, \ldots, p^{K}, \nabla c\left(p^{1}\right) u_{1}, \ldots, \nabla c\left(p^{K}\right) u_{K}\right] \\
=u_{i} / u_{j} ; \quad 1 \leq i<j \leq K .
\end{gathered}
$$

In the above definition of exactness, we are assuming optimizing behavior, with quantities $y^{k}$ in the share functions $S^{k}(P, Y)$ being replaced by the Hicksian demand functions $\nabla c\left(p^{k}\right) u_{k}$; see (9) above. Thus the strictly positive country price vectors $p^{k}>>0_{N}$ and the positive country utility levels $u_{k}>0$ are regarded as the independent variables in the system of functional equations defined by (13). 
In analogy with the economic approach to bilateral index number theory, we would like a given multilateral system of share functions $S^{k}(P, Y)$ to be exact for a flexible functional form for either: (i) the homogeneous aggregator function $f$ that appears in (12) or (ii) the unit cost function $c$ that appears in (13). This exactness property for a multilateral system is a minimal property (from the viewpoint of economic theory) that the system should possess. If this property is not satisfied, then the multilateral system is consistent only with aggregator functions that substantially restrict substitution possibilities between commodities. If the multilateral system $S^{k}(P, Y)$ does have the above exactness property for either case (i) or (ii), we say that the multilateral system is superlative. This is a straightforward generalization of the idea of a superlative bilateral ${ }^{12}$ index number formula to the multilateral context.

In the following 10 sections, we shall evaluate many of the commonly used multilateral systems with respect to the 12 tests listed in section 2 . We shall also determine whether each multilateral system is superlative.

\section{The Exchange Rate Method}

The first multilateral method that we consider is the simplest: all country prices are converted into a common currency (our country price vectors $p^{k}$ have already incorporated this conversion to a numeraire currency) and the share function for country $k, S^{k}(P, Y)$, is defined to be its nominal share of bloc output, $p^{k} \cdot y^{k} / \Sigma_{j=1}^{K} p^{j} \cdot y^{j}$; equations (1) in section 2 above.

Proposition 1: The exchange rate method passes tests $T 1, T 4, T 5, T 6, T 8$ and $T 9$ and fails the remaining 6 tests. The exchange rate method is not exact for any aggregator function or any unit cost function and hence is not a superlative method.

Proofs of Propositions are in Appendix 1.

Proposition 1 shows that the exchange rate method has very poor axiomatic and economic properties. However, due to its simplicity and minimal data requirements (only domestic value information plus exchange rate information is required), it is probably the most commonly used method for making multilateral comparisons.

We turn now to a class of additive methods. 


\section{Symmetric Mean Average Price Methods}

Recall the definition of an additive multilateral method defined by (3) and (4) above. In this section, we shall assume that the weighting functions $g_{n}(P, Y)$ are averages of country prices for commodity $n, p_{n}^{1}, \ldots, p_{n}^{K}$, for $n=1, \ldots, N$. Specifically, we assume that

$$
g_{n}(P, Y) \equiv m\left(p_{n}^{1}, p_{n}^{2}, \ldots, p_{n}^{K}\right) ; \quad n=1, \ldots, N
$$

where $m$ is a homogeneous symmetric mean. ${ }^{13}$ Two special cases for $m$ are the arithmetic and geometric means, defined by (15) and (16) respectively:

$$
\begin{array}{ll}
g_{n}(P, Y) \equiv \Sigma_{k=1}^{K}(1 / K) p_{n}^{k} ; & n=1, \ldots, N \\
g_{n}(P, Y) \equiv\left[\Pi_{k=1}^{K} p_{n}^{k}\right]^{1 / K} ; & n=1, \ldots, N .
\end{array}
$$

The geometric average price multilateral system defined by (3) and (16) was originally suggested by Walsh [1901; 381 and 398] (his double weighting method), noted by Gini $[1924 ; 106]$ and implemented by Gerardi [1982; 387]. It turns out that this method satisfies more tests than other symmetric mean average price methods.

Proposition 2: The general symmetric mean average price multilateral method defined by (3) and (14) (but excluding (16)) satisfies all tests except the monetary units test $T 7$ and the two country weighting tests $T 10$ and $T 11$. The geometric average price method defined by (3) and (16) satisfies all tests except T10 and T11. Symmetric mean average price methods are exact only for the linear aggregator function $f$ defined by (17) and the linear unit cost function $c$ defined by (18) below. Hence these methods are not superlative.

A linear aggregator function $f$ is defined as

$$
f\left(y_{1}, \ldots, y_{N}\right) \equiv \Sigma_{n=1}^{N} a_{n} y_{n}
$$

where the the parameters $a_{n}$ are positive. A linear unit cost function $c$ (dual to a Leontief no substitution aggregator function) is defined as

$$
c\left(p_{1}, \ldots, p_{N}\right) \equiv \Sigma_{n=1}^{N} b_{n} p_{n}
$$

where the parameters $b_{n}$ are positive.

From Proposition 2, we see that the geometric average price method is quite a good one from the axiomatic perspective: the method fails only the two consistency in aggregation tests $T 10$ and $T 11$. However, from the economic perspective, the Gerardi-Walsh geometric average price method is not satisfactory: it is consistent only with aggregator 
functions that exhibit perfect substitutability (see (17) above) or complete nonsubstitutability (see (18) above).

Instead of using average prices to define additive quantity indexes, average quantities could be used to define additive price indexes (or purchasing power parities as they are called in the multilateral literature). We turn now to the consideration of this third class of multilateral methods.

\section{Symmetric Mean Average Quantity Methods}

For this class of methods, we first define country $k$ 's price level $P^{k}$ as follows:

$$
P^{k}(P, Y) \equiv \Sigma_{n=1}^{N} m\left(y_{n}^{1}, \ldots, y_{n}^{K}\right) p_{n}^{k} ; \quad k=1, \ldots, K
$$

where $m$ is a homogeneous symmetric mean. If we define $\bar{y}_{n} \equiv m\left(y_{n}^{1}, \ldots, y_{n}^{K}\right)$ as an average over countries of commodity $n$, then we see that country $k$ 's price level $P^{k}$ is simply the value of the average basket $\left[\bar{y}_{1}, \ldots, \bar{y}_{N}\right]^{T} \equiv \bar{y}$ evaluated using the prices of country $k,\left[p_{1}^{k}, \ldots, p_{N}^{k}\right]^{T} \equiv p^{k}$.

Once the price levels $P^{k}$ have been defined, the corresponding country $k$ quantity levels ${ }^{14} Q^{k}$ can be defined residually using the following equations:

$$
P^{k} Q^{k}=p^{k} \cdot y^{k} ; \quad k=1, \ldots, K
$$

i.e., aggregate price times quantity for country $k$ should equal the value of country $k$ consumption (or production or input), $p^{k} \cdot y^{k}$. Finally, given the quantity levels $Q^{k}$, they can be normalized into shares $S^{k}$ :

$$
\begin{aligned}
S^{k} & \equiv Q^{k} / \Sigma_{i=1}^{K} Q^{i} \\
& =\left[p^{k} \cdot y^{k} / p^{k} \cdot \bar{y}\right] /\left[\Sigma_{k=1}^{K} p^{i} \cdot y^{i} / p^{i} \cdot \bar{y}\right]
\end{aligned}
$$

where (22) follows by substituting (19) and (20) into (21). Recall that $\bar{y}$ is the average quantity vector which has $n$th component equal to $\bar{y}_{n} \equiv m\left(y_{n}^{1}, \ldots, y_{n}^{K}\right)$.

As in the previous section, two special cases for the homogeneous symmetric mean $m$ that appeared in (19) are of interest: the arithmetic and geometric means defined by (23) and (24):

$$
\begin{aligned}
& \bar{y}_{n}=m\left(y_{n}^{1}, \ldots, y_{n}^{K}\right) \equiv \Sigma_{k=1}^{K}(1 / K) y_{n}^{k} ; \quad n=1, \ldots, N ; \\
& \bar{y}_{n}=m\left(y_{n}^{1}, \ldots, y_{n}^{K}\right) \equiv\left[\Pi_{k=1}^{K} y_{n}^{k}\right]^{1 / K} ; \quad n=1, \ldots, N
\end{aligned}
$$


Walsh $[1901 ; 431]$ called the multilateral method defined by (22) and (23) "Scrope's method with arithmetic weights" while Fisher [1922; 307] called it the "broadened base system" and Gini $[1931 ; 8]$ called it the "standard population method". Walsh [1901; 398] called the multilateral method defined by (22) and (24) "Scrope's further emended method with geometric weights". This index was later independently advocated by Gerardi $[1982 ; 389]$.

The following Proposition shows that average quantity methods satisfy fewer multilateral tests than average price methods but they have equivalent exactness properties.

Proposition 3: A symmetric mean average quantity multilateral method defined by (22) and $\bar{y}_{n} \equiv m\left(y_{n}^{1}, \ldots, y_{n}^{K}\right), n=1, \ldots, N$ where $m$ is a general homogeneous symmetric mean (excluding the two special cases (23) and (24)) satisfies tests $T 1-T 7$ and fails tests $T 8$ and $T 10-T 12$. The geometric weights method defined by (22) and (24) passes tests $T 1-T 8$ and fails tests $T 9-T 12$. The arithmetic weights method defined by (22) and (23) passes tests $T 1-T 7$ and $T 9$ and fails $T 8$ and $T 10-T 12$. Symmetric mean average quantity methods are exact for only the linear aggregator function $f$ defined by (17) and the linear unit cost function $c$ defined by (18). Hence these methods are not superlative.

Note that the above Proposition does not determine whether the monotonicity test $T 9$ holds for a general homogeneous symmetric mean: we were only able to determine that the linear mean method defined by (23) satisfies $T 9$ but the geometric mean method defined by (24) does not satisfy $T 9$.

Comparing Propositions 2 and 3, we see that the Gerardi-Walsh geometric average price method defined by (3) and (16) dominates all of the methods defined in this section and the previous section, failing only the two country weighting tests $T 10$ and $T 11$.

We turn now to a more complex average price method.

\section{The Geary-Khamis Average Price Method}

The basic equations defining the Geary-Khamis ${ }^{15}$ method can be set out as follows. Define an average price for commodity $n$ by

$$
\pi_{n} \equiv \Sigma_{k=1}^{K}\left[y_{n}^{k} / \Sigma_{j=1}^{K} y_{n}^{j}\right]\left[p_{n}^{k} / P^{k}\right] ; \quad n=1, \ldots, N
$$

where the country $k$ price level or purchasing power parity $P^{k}$ is defined as

$$
P^{k} \equiv p^{k} \cdot y^{k} / \pi \cdot y^{k} ; \quad k=1, \ldots, K
$$


where $\pi \equiv\left[\pi_{1}, \ldots, \pi_{N}\right]^{T}$ is the vector of Geary-Khamis bloc average prices. Note that $\pi_{n}$ is a weighted average of the purchasing power parity adjusted country prices $p_{n}^{k} / P^{k}$ for commodity $n$, where the country $k$ weight is equal to its share of the total quantity of commodity $n, y_{n}^{k} / \Sigma_{j=1}^{K} y_{n}^{j}$. Once the $\pi_{n}$ and $P^{k}$ have been determined by (25) and (26), the country $k$ quantity levels $Q^{k}$ and shares $S^{k}$ can be determined using equations (20) and (21).

If we substitute equations (26) into (25), the equations which define the Geary-Khamis share functions can be simplified into the following system of equations:

$$
\begin{aligned}
{\left[I_{N}-C\right] \pi } & =0_{n} ; \\
y \cdot \pi & =1 ; \\
S^{k} & =\pi \cdot y^{k} ; \quad k=1, \ldots, K
\end{aligned}
$$

where $I_{N}$ is the $N$ by $N$ identity matrix, $y \equiv \Sigma_{k=1}^{K} y^{k}>>0_{N}$ is the strictly positive bloc total quantity vector and the strictly positive $N$ by $N$ matrix $C$ is defined by

$$
C \equiv \hat{y}^{-1} \Sigma_{k=1}^{K} \hat{p}^{k} y^{k} y^{k T} / p^{k} \cdot y^{k}
$$

where $\hat{p}^{k}$ is the country $k$ positive price vector $p^{k}>>0_{N}$ diagonalized into a matrix and $\hat{y}$ is the total quantity vector $y \equiv \Sigma_{k=1}^{K} y^{k}$ diagonalized into a matrix.

Using (30), note that $y^{T} C=y^{T}$. Thus the positive vector $y$ is a left eigenvector of the positive matrix $C$ that corresponds to a unit eigenvalue. Hence by the Perron [1907; 46] - Frobenius $[1909 ; 514]^{16}, \lambda=1$ is the maximal eigenvalue of $C$ and $C$ also has a strictly positive right eigenvector $\pi$ that corresponds to this maximal eigenvalue; i.e., we have the existence of $\pi>0_{N}$ such that $C \pi=\pi$ which is (27). This positive right eigenvector can then be normalized to satisfy (28). From (29), we see that the Geary-Khamis method satisfies the additivity test $T 12$.

The following Proposition shows that the Geary-Khamis multilateral system does rather well from the viewpoint of the axiomatic approach but not so well from the viewpoint of the economic approach.

Proposition 4: The Geary-Khamis multilateral system of share functions defined by (27) - (30) satisfies all of the multilateral tests except $T 8$ (homogeneity in quantities), $T 9$ (monotonicity in quantities) and T11 (bilateral consistency in aggregation). However, the Geary-Khamis method does satisfy a reasonable modification of $T 11$. The method is exact only for the linear aggregator function $f$ defined by (17) and the linear unit cost function $c$ defined by (18). Hence the method is not superlative. 
Proponents of the $G K$ system might argue that the method's failure with respect to test $T 11$ is perhaps exaggerated, since instead of ending up with a bilateral Fisher quantity index $Q_{F}$ under the conditions of test $T 11$, we end up with the bilateral GK quantity index $Q_{G K}$; i.e., under the conditions of test $T 11$, we obtain:

$$
\Sigma_{j \in B} S^{j}(P, Y) / \Sigma_{i \in A} S^{i}(P, Y)=p^{b} \cdot y^{b} / p^{a} \cdot y^{a} P_{G K}\left(p^{a}, p^{b}, y^{a}, y^{b}\right)
$$

where the $G K$ bilateral price index $P_{G K}$ is defined by ${ }^{17}$

$$
P_{G K}\left(p^{a}, p^{b}, y^{a}, y^{b}\right) \equiv \Sigma_{n=1}^{N} h\left(y_{n}^{a}, y_{n}^{b}\right) p_{n}^{b} / \Sigma_{m=1}^{N} h\left(y_{n}^{a}, y_{n}^{b}\right) p_{n}^{a}
$$

and where $h(x, z) \equiv 2 x z /[x+z]$ is the harmonic mean of $x$ and $\left.z,[(1 / 2)] x^{-1}+(1 / 2) z^{-1}\right]^{-1}$, if both $x$ and $z$ are positive. However, from the viewpoint of the test approach to bilateral index number theory, the Fisher price and quantity indexes pass considerably more tests than the Geary-Khamis price and quantity indexes. The Fisher bilateral price index satisfies all 20 of the tests listed in Diewert $[1992 ; 214-221]^{18}$ while the Geary-Khamis bilateral price index fails 6 of these tests: PT7 (homogeneity of degree 0 in current period quantities), PT8 (homogeneity of degree 0 in base period quantities), PT13 (price reversal or price weights symmetry), PT16 (the Paasche and Laspeyres bounding test), PT19 (monotonicity in base period quantities) and PT20 (monotonicity in current period quantities). The failure of bilateral test PT13 is not important but the failure of the other tests is troublesome.

From the viewpoint of the economic approach to index number theory, the GearyKhamis method is definitely inferior to the multilateral systems that will be discussed later in sections 10-13. Note that even in the two country case $(K=2)$, the $G K$ method is exact only for the linear aggregator function (17) and the linear unit cost function (18). Thus the method is consistent only with perfect substitutability or with perfect nonsubstitutability.

\section{Van Yzeren's Unweighted Average Price Method}

In this method, a vector of bloc average prices $p^{*}$ is defined in a manner similar to the definition of the Geary-Khamis average price vector $\pi$ (recall (25) above) except that the price vector of each country $p^{k}$ divided by its purchasing power parity or price level $P^{k}$ is weighted equally. Van Yzeren $[1957 ; 13]$ originally called this method the homogeneous group method. He later called it (Van Ijzeren [1983; 40]) a price combining method or an unweighted international price method. The equations defining this method are (33) - (36) below:

$$
p^{*} \equiv \alpha \Sigma_{k=1}^{K} p^{k} / P^{k}
$$




$$
\begin{array}{lll}
\text { (34) } & P^{k} \equiv p^{k} \cdot y^{k} / p^{*} \cdot y^{k} ; & k=1, \ldots, K ; \\
\text { (35) } & S^{k} \equiv p^{*} \cdot y^{k} ; & k=1, \ldots, K ; \\
\text { (36) } & \Sigma_{k=1}^{K} S^{k}=1, &
\end{array}
$$

where $\alpha$ is a positive number. If we substitute (34) into (33) and substitute (35) into (36), we find that the vector of bloc average prices $p^{*}$ and the scalar $\alpha$ must satisfy the following two equations;

$$
\begin{aligned}
& p^{*}=\alpha\left[\Sigma_{k=1}^{K}\left(p^{k} \cdot y^{k}\right)^{-1} p^{k} y^{k T}\right] p^{*} \equiv \alpha C p^{*} \\
& 1=\left[\Sigma_{k=1}^{K} y^{k}\right] \cdot p^{*}=y \cdot p^{*}
\end{aligned}
$$

where $C \equiv\left[c_{i j}\right]$ with $c_{i j} \equiv \Sigma_{k=1}^{K} p_{i}^{k} y_{j}^{k} / p^{k} \cdot y^{k}$ and $y \equiv \Sigma_{k=1}^{K} y^{k}$ is the bloc total quantity vector as usual. Since $y^{k}>0_{N}$ and $p^{k}>>0_{N}$ for each $k$ with $\Sigma_{k=1}^{K} y^{k}>>0_{N}, C$ is a matrix with positive elements. Hence $\alpha=1 / \lambda>0$ where $\lambda$ is the largest positive eigenvalue of $C$ and $p^{*}>>0_{N}$ is a normalization of the corresponding strictly positive right eigenvector of $C$ (recall the Perron-Frobenius Theorem). Thus if the number of goods $N$ is equal to 2 , it is possible to work out an explicit algebraic formula for the $S^{k}$.

It is possible to express the defining equations for this method in a different manner which will give some additional insight. Substitute (34) into (33) and premultiply the resulting (33) by $y^{i T}$ for $i=1, \ldots, K$. Using (35), the resulting $K$ equations become

$$
S^{i}=\alpha \Sigma_{j=1}^{K}\left(p^{j} \cdot y^{j}\right)^{-1} p^{j} \cdot y^{i} S^{j} ; \quad i=1, \ldots, K
$$

After defining the vector of shares $s \equiv\left[S^{1}, \ldots, S^{K}\right]^{T}$, equations (39) can be rewritten using matrix notation as

$$
s=\alpha D s
$$

where the $i j$ th element of the $K$ by $K$ matrix $D$ is defined as $d_{i j} \equiv p^{j} \cdot y^{i} / p^{j} \cdot y^{j}>0$ for $i, j=$ $1, \ldots, K$. Since $D$ is positive, take $\alpha=1 / \lambda$ where $\lambda$ is the maximal positive eigenvalue of $D$ and $s$ is a normalization of the corresponding strictly positive right eigenvector of $D .^{19}$ The definition of $s$ and $\alpha$ using equations (36) and (40) is the way Van Yzeren [1957; 13] originally defined his homogeneous group method. We have used the techniques of Van Ijzeren $[1983 ; 40-41]$ to show that (36) and (40) are equivalent to (33) - (36).

Before we summarize the properties of Van Yzeren's unweighted homogeneous group method in Proposition 5 below, it will be useful to note that the following flexible functional forms are exact ${ }^{20}$ for the Fisher ideal quantity index $Q_{F}$ defined above by (2):

$$
f(y) \equiv\left(y^{T} A y\right)^{\frac{1}{2}} ; \quad A=A^{T}
$$




$$
c(p) \equiv\left(p^{T} B p\right)^{\frac{1}{2}} ; \quad B=B^{T} .
$$

The $f$ defined by (41) is the square root quadratic aggregator function and the cost function defined by (42) is the square root quadratic unit cost function. If either of the matrices $A$ or $B$ has an inverse, then $A=B^{-1}$.

Proposition 5: Van Yzeren's Unweighted Average Price Method defined by (36) and (40) satisfies all of the multilateral tests except $T 9$ (monotonicity) and the two consistency in aggregation tests $T 10$ and $T 11$. For $K \geq 3$, this method is exact only for the linear aggregator function defined by (17) and the linear unit cost function defined by (18). However, for the two country case $(K=2)$, this method is exact for the $f$ defined by (41) and the $c$ defined by (42). Finally, in the $K=2$ case, $S^{2} / S^{1}=Q_{F}\left(p^{1}, p^{2}, y^{1}, y^{2}\right)$ where $Q_{F}$ is the Fisher ideal quantity index defined by (2).

Proposition 5 shows that this average price method suffers from the same limitation possessed by the average price methods studied in section 5 and 7 : when $K \geq 2$, these methods are consistent only with perfect substitutability or zero substitutability.

Note that Van Yzeren's unweighted average price method (which fails $T 9-T 11$ ) is dominated by the Gerardi-Walsh geometric mean average price method (which fails only $T 10$ and $T 11$ ) discussed earlier in section 5.

We turn now to an analysis of the average quantity counterpart to the present method.

\section{Van Yzeren's Unweighted Average Basket Method}

In this method, a vector of bloc average quantities $y^{*}$ is defined in a manner that is analogous to the definition of the average prices $p^{*}$ in the previous section, except that the role of prices and quantities is interchanged. Van Yzeren $[1957 ; 6-14]$ originally called this method the heterogeneous group method and he later (Van Ijzeren [1983; 40-44]) called it an unweighted basket combining method.

$$
\begin{aligned}
& y^{*}=\alpha \Sigma_{k=1}^{K} y^{k} / S^{k} \\
& P^{k}=p^{k} \cdot y^{*} ; \quad k=1, \ldots, K \\
& P^{k} S^{k}=p^{k} \cdot y^{k} ; \quad k=1, \ldots, K \\
& \Sigma_{k=1}^{K} S^{k}=1 .
\end{aligned}
$$

If we substitute (44) and (45) into (43) and (46), we find that the vector of bloc average quantities $y^{*}$ and the scalar $\alpha$ must satisfy the following $N+1$ equations: 


$$
\begin{aligned}
& y^{*}=\alpha\left[\Sigma_{k=1}^{K}\left(p^{k} \cdot y^{k}\right)^{-1} y^{k} p^{k T}\right] y^{*}=\alpha C^{T} y^{*} \\
& 1=\Sigma_{k=1}^{K} p^{k} \cdot y^{k} / p^{k} \cdot y^{*}
\end{aligned}
$$

where $C \equiv\left[c_{i j}\right]$ with $c_{i j} \equiv \Sigma_{k=1}^{K} p_{i}^{k} y_{j}^{k} / p^{k} \cdot y^{k}$ is the same matrix which appeared earlier in (37). We can satisfy (47) by choosing $\alpha=1 / \lambda$ where $\lambda$ is the maximum positive eigenvalue of the positive matrix $C$ and by choosing $y^{*}$ to be a normalization of the corresponding positive left eigenvector of $C$ (or positive right eigenvector of $C^{T}$ ). The normalization of the eigenvector is determined by (48). As in the previous section, if the number of commodities $N$ is equal to two, then it is possible to work out an explicit formula for the $S^{k}$.

As in the previous section, it is useful to transform the above equations into a more useful form. For $i=1, \ldots, K$, premultiply both sides of (43) by $p^{i T}$. Using (44) and (45), the resulting system of equations can be written as

$$
\left(S^{i}\right)^{-1}=\alpha \Sigma_{j=1}^{K}\left[p^{i} \cdot y^{j} / p^{i} \cdot y^{i}\right]\left(S^{j}\right)^{-1} ; \quad i=1, \ldots, K .
$$

Define the vector $s^{-1} \equiv\left[\left(S^{1}\right)^{-1},\left(S^{2}\right)^{-1}, \ldots,\left(S^{K}\right)^{-1}\right]^{T}$. Then equations (49) can be written in matrix form as

$$
s^{-1}=\alpha D^{T} s^{-1}
$$

where $D^{T}$ is the transpose of the matrix $D$ defined in the previous section below (40). Thus as in the previous section, we can take $\alpha=1 / \lambda$ where $\lambda$ is the maximum positive eigenvalue of the positive matrix $D$ and in this section, we let $s^{-1}$ be proportional to the positive left eigenvector of $D$ that corresponds to $\lambda$, the factor of proportionality being determined by (46).

Van Yzeren $[1957 ; 25]$ initially defined his heterogeneous group method using a version of (50), except that the $S^{k}$ in (50) were replaced by the parities $P^{k}$ using equations (45). Later, Van Ijzeren $[1983 ; 40]$ derived the average basket interpretation of this method that was defined by (43) - (46) above.

Proposition 6: Van Yzeren's Unweighted Average Basket Method defined by (46) and (50) satisfies all of the multilateral tests except the monotonicity test $T 9$, the two consistency in aggregation tests $T 10$ and $T 11$ and the additivity test $T 12$. For $K \geq 3$, the method is exact only for the linear aggregator function defined by (17) and the linear unit cost function defined by (18). However, for the two country case $(K=2)$, the method is exact for the $f$ defined by (41) and the $c$ defined by (42) and in this case, $S^{2} / S^{1}=Q_{F}$ is the Fisher ideal quantity index defined by (2). 
Proposition 6 shows that Van Yzeren's average basket method suffers from the same limitation that applied to all of the methods studied in sections 5-8 above: if $K \geq 3$, these methods are consistent only with perfect substitutability or zero substitutability.

The average basket method (which fails $T 9-T 12$ ) is dominated by Van Yzeren's average price method (which fails $T 9-T 11$ ) and the Gerardi-Walsh method (which fails only $T 10-T 11)$.

The multilateral methods of Van Yzeren presented in this section and the previous section are generalizations of the bilateral Fisher ideal quantity index in the sense that these methods reduce to the Fisher index when there are only 2 countries. However, these methods are not very satisfactory generalizations in the 3 or more country case because these methods are not exact for the flexible functional forms defined by (41) and (42). The multilateral methods that will be discussed in the following 4 sections do not suffer from this inflexibility: the methods which follow are all exact for the $f$ defined by (41) and the $c$ defined by (42) and hence are superlative. Moreover, all of the multilateral methods which follow can be viewed as methods which attempt to harmonize the inconsistent comparisons that are generated by using a bilateral quantity index $Q$ in the multilateral context.

\section{The Gini-EKS System}

We turn now to an examination of a multilateral method that uses a bilateral price or quantity index, $P\left(p^{i}, p^{j}, y^{i}, y^{j}\right)$ or $Q\left(p^{i}, p^{j}, y^{i}, y^{j}\right)$, as the basic building bloc. We assume for the remainder of the paper that the bilateral price and quantity index satisfy ${ }^{21}$

$$
P\left(p^{i}, p^{j}, y^{i}, y^{j}\right) Q\left(p^{i}, p^{j}, y^{i}, y^{j}\right)=p^{j} \cdot y^{j} / p^{i} \cdot y^{i} .
$$

Thus if $P$ is given, then the corresponding $Q$ can be defined via (51) and vice versa.

Suppose that the bilateral quantity index $Q$ satisfies Fisher's $[1922 ; 413]$ circularity test $t^{22}$; i.e., for every set of three price and quantity vectors, we have

$$
Q\left(p^{1}, p^{2}, q^{1}, q^{2}\right) Q\left(p^{2}, p^{3}, q^{2}, q^{3}\right)=Q\left(p^{1}, p^{3}, q^{1}, q^{3}\right)
$$

We shall show why circularity is a useful property in the context of making multilateral comparisons shortly.

It is obvious that a bilateral quantity index $Q$ can be used to generate a multilateral system of share functions provided that we are willing to asymmetrically single out one country to play the role of a base country. For example, suppose we have a bilateral $Q$ and we choose country 1 to be the base country. Then the share of country $k, S_{k}$ say, relative to the share of country $1, S_{1}$ say, can be defined as follows ${ }^{23}$ : 


$$
S_{k} / S_{1} \equiv Q\left(p^{1}, p^{k}, y^{1}, y^{k}\right) ; \quad k=1, \ldots, K
$$

Equations (53) and the normalizing equation

$$
\Sigma_{k=1}^{K} S_{k}=1
$$

will determine the multilateral shares using country 1 as the base. ${ }^{24}$

The problem with the multilateral star method defined by (53) and (54) is that, in general, the method will not satisfy test $T 6$; i.e., the method will not be independent of the choice of the base country. However, if the bilateral quantity index $Q$ satisfies the circularity test (52), then the star system would be independent of the base country. We demonstrate this assertion as follows. Consider the multilateral shares $S_{k}^{*}$ that are generated by $Q$ using country 2 as the base:

$$
S_{k}^{*} / S_{2}^{*} \equiv Q\left(p^{2}, p^{k}, y^{2}, y^{k}\right) ; \quad k=1, \ldots, K
$$

Now assume that $Q$ satisfies circularity (52) and premultiply both sides of (55) by the constant $S_{2} / S_{1} \equiv Q\left(p^{1}, p^{2}, y^{1}, y^{2}\right)$ :

$$
\begin{array}{rlrl}
{\left[S_{2} / S_{1}\right]\left[S_{k}^{*} / S_{2}^{*}\right]} & =Q\left(p^{1}, p^{2}, y^{1}, y^{2}\right) Q\left(p^{2}, p^{k}, y^{2}, y^{k}\right) \\
& =Q\left(p^{1}, p^{k}, y^{1}, y^{k}\right) & & \text { using (52) } \\
& =S_{k} / S_{1} & & \text { using (53). }
\end{array}
$$

Thus the $S_{k}$ are proportional to the $S_{k}^{*}$ and hence, after using the normalization (54), they must be identical.

Unfortunately, if the bilateral index $Q$ satisfies the circularity test for all price and quantity vectors, then Eichhorn $[1976][1978 ; 162-169]$ and Balk $[1995 \mathrm{a} ; 75-77]$ show that $Q$ does not satisfy many other reasonable bilateral tests. In fact, Eichhorn's methods may be used to prove the following result.

Proposition 7: Suppose that the bilateral quantity index $Q$ satisfies the circularity test (52) and the following bilateral tests: BT1 (positivity), BT3 (identity), BT5 (proportionality in current period quantities), BT10 (commensurability), and BT12 (monotonicity in current period quantities). Then $Q\left(p^{1}, p^{2}, y^{1}, y^{2}\right)=\Pi_{n=1}^{N}\left(y_{n}^{2} / y_{n}^{1}\right)^{\alpha_{n}}$ where the $\alpha_{n}$ are positive constants summing to unity.

The bilateral tests BT1 -BT13 will be defined later in this section. Proposition 7 merely illustrates that Irving Fisher's $[1922 ; 274]$ intuition was correct: if a bilateral quantity index ${ }^{25}$ satisfies the circular test plus a few other reasonable tests, then the index must have constant price weights ${ }^{26}$ which leads to nonsensical results. ${ }^{27}$ Thus as a practical matter, we cannot appeal to circularity to make the star system a symmetric method. 
Returning to the asymmetric star system defined by (53) and (54), if instead of country 1 , we use country $i$ as the base, then the share of country $k$ using $i$ as a base, $S_{k}^{(i)}$, can be defined using the bilateral quantity index $Q$ as follows:

$$
S_{k}^{(i)} \equiv Q\left(p^{i}, p^{k}, y^{i}, y^{k}\right) / \Sigma_{j=1}^{K} Q\left(p^{i}, p^{j}, y^{i}, y^{j}\right) ; \quad i, k=1, \ldots, K .
$$

Fisher $[1922 ; 305]$ was perhaps the first to realize that the asymmetric multilateral methods defined by (56) could be made to satisfy the symmetric treatment of countries tests $T 6$ by taking the arithmetic mean of the shares defined by (56); i.e., the Fisher blended share ${ }^{28}$ for country $k, S_{k}^{F}$, can be defined by equations (57):

$$
S_{k}^{F} \equiv \Sigma_{i=1}^{K}(1 / K) S_{k}^{(i)} ; \quad k=1, \ldots, K
$$

Instead of using an arithmetic average of the $S_{k}^{(i)}$ defined by $(56)$, Gini $[1924 ; 110]$ [1931; 12] proposed using a geometric average. Thus the Gini share of bloc aggregate quantity for country $k$ turns out to be proportional to $\left[Q\left(p^{1}, p^{k}, y^{1}, y^{k}\right) \ldots Q\left(p^{K}, p^{k}, y^{K}, y^{k}\right)\right]^{(1 / K)}$; i.e.,

$$
S_{k}^{G} \equiv \alpha\left[\Pi_{i=1}^{K} Q\left(p^{i}, p^{k}, y^{i}, y^{k}\right)\right]^{(1 / K)} ; \quad k=1, \ldots, K
$$

where $\alpha$ is chosen so that the $S_{k}^{G}$ sum to one. In general, Gini [1931; 10] required only that his bilateral index number formula ${ }^{29}$ satisfy the time reversal test; i.e., that $Q\left(p^{2}, p^{1}, y^{2}, y^{1}\right)=1 / Q\left(p^{1}, p^{2}, y^{1}, y^{2}\right)$. In his empirical work, Gini [1931; $\left.13-24\right]$ used the Fisher ideal formula. Finally, Gini $[1931 ; 10]$ called his multilateral method the circular weight system. Gini's method, using the Fisher ideal formula, was later independently proposed by Eltetö and Köves [1964] and Szulc [1964] and is known as the EKS system.

Eltetö and Köves and Szulc actually derived their multilateral system ( 58 ) by a different route which we shall now explain. Let $P_{k}$ be the country $k$ price level that corresponds to country $k$ 's multilateral share $S_{k}$. As usual, we impose the following restriction on the $P_{k}$ and $S_{k}$ :

$$
P_{k} S_{k}=p^{k} \cdot y^{k} ; \quad k=1, \ldots, K
$$

Now pick bilateral price and quantity indexes, $P$ and $Q$, that satisfy the product test (51). The country price levels $P_{k}$ are determined by solving the following least squares problem:

$$
\begin{array}{rlr} 
& \min _{P_{1}, \ldots, P_{K}} \Sigma_{i=1}^{K} \Sigma_{j=1}^{K}\left\{\ln \left[\left(P_{i} / P_{j}\right) P\left(p^{i}, p^{j}, y^{i}, y^{j}\right)\right]\right\}^{2} & \\
= & \min _{P_{1}, \ldots, P_{K}} \Sigma_{i=1}^{K} \Sigma_{j=1}^{K}\left\{\ln \left[\left(P_{i} / P_{j}\right) p^{j} \cdot y^{j} / p^{i} \cdot y^{i} Q\left(p^{i}, p^{j}, y^{i}, y^{j}\right)\right]\right\}^{2} & \text { using (51) } \\
= & \min _{S_{1}, \ldots, S_{K}} \Sigma_{i=1}^{K} \Sigma_{j=1}^{K}\left\{\ln \left[\left(S_{j} / S_{i}\right) / Q\left(p^{i}, p^{j}, y^{i}, y^{j}\right)\right]\right\}^{2} & \text { using (59) } \\
= & \min _{S_{1}, \ldots, S_{K}} \Sigma_{i=1}^{K} \Sigma_{j=1}^{K}\left\{\ln \left[\left(S_{j} / S_{i}\right) Q\left(p^{j}, p^{i}, y^{j}, y^{i}\right)\right]\right\}^{2} &
\end{array}
$$


where (61) follows from the line above if $Q$ satisfies the time reversal test. Thus if the bilateral quantity index $Q$ satisfies the time reversal test, finding the optimal price levels $P_{k}$ that solve the least squares problem (60) is equivalent to finding the optimal country shares $S_{k}$ that solve the least squares problem (61). Note that the objective function in $(60)^{2}$ is homogeneous of degree zero in the $P_{k}$ and the objective function in (61) is homogeneous of degree zero in the $S_{k}$. Hence a normalization on the $P_{k}$ or $S_{k}$ is required to determine their absolute levels. As usual, we choose the normalization (54).

Differentiating the objective function in (61) with respect to $S_{k}$ leads to the following equations for $k=1, \ldots, K$ :

$$
\begin{aligned}
\ln S_{k}-(1 / K) \Sigma_{j=1}^{K} \ln S_{j} & =(1 / 2 K) \Sigma_{j=1}^{K} \ln Q\left(p^{j}, p^{k}, y^{j}, y^{k}\right) \\
& -(1 / 2 K) \Sigma_{i=1}^{K} \ln Q\left(p^{k}, p^{i}, y^{k}, y^{i}\right)
\end{aligned}
$$

If $Q$ satisfies the time reversal test ${ }^{30}$, then equations (62) simplify to $^{31}$

$$
S_{k} /\left[S_{1} \ldots S_{K}\right]^{1 / K}=\left[\Pi_{j=1}^{K} Q\left(p^{j}, p^{k}, y^{j}, y^{k}\right)\right]^{1 / K} ; \quad k=1, \ldots, K
$$

Using the normalization (54), it can be seen that the shares defined by (63) and (54) are identical to the Gini shares defined by (58) and (54).

Eltetö and Köves [1964] and Szulc [1964] used the least squares problem (60) with $P$ equal to the Fisher ideal bilateral price index $P_{F}$ to derive the $E K S$ purchasing power parities $P_{k}$. Van Ijzeren $[1987 ; 62-65]$ showed that one also obtained the $E K S P_{k}$ and $S_{k}$ if the Fisher, Paasche or Laspeyres price indexes were used as the $P$ in (60) or if the Fisher, Paasche or Laspeyres quantity indexes were used as the $Q$ in (61). ${ }^{32}$ We shall call the system of shares defined by (54) and (58) for a general bilateral $Q$ satisfying the time reversal test the Gini System. When $Q$ is set equal to the bilateral Fisher ideal quantity index $Q_{F}$, we call the system defined by (54) and (58) the Gini-EKS System.

In order to determine the axiomatic properties of the Gini system, we shall assume that the bilateral quantity index satisfies the following 13 bilateral tests: ${ }^{33}$

BT1: Positivity: $Q\left(p^{1}, p^{2}, y^{1}, y^{2}\right)>0$.

BT2: Continuity: $Q$ is a continuous function of its arguments.

BT3: Identity: $Q\left(p^{1}, p^{2}, y, y\right)=1$

BT4: $\quad$ Constant Prices: $\quad Q\left(p, p, y^{1}, y^{2}\right)=p \cdot y^{2} / p \cdot y^{1}$

BT5: Proportionality in Current Period Quantities: $\quad Q\left(p^{1}, p^{2}, y^{1}, \lambda y^{2}\right)=\lambda Q\left(p^{1}, p^{2}, y^{1}, y^{2}\right)$ for all $\lambda>0$. 
BT6: Inverse Proportionality in Base Period Quantities: $\quad Q\left(p^{1}, p^{2}, \lambda y^{1}, y^{2}\right)=$ $\lambda^{-1} Q\left(p^{1}, p^{2}, y^{1}, y^{2}\right)$ for all $\lambda>0$.

BT7: Homogeneity in Current Period Prices: $\quad Q\left(p^{1}, \lambda p^{2}, y^{1}, y^{2}\right)=Q\left(p^{1}, p^{2}, y^{1}, y^{2}\right)$ for all $\lambda>0$.

BT8: Homogeneity in Base Period Prices: $\quad Q\left(\lambda p^{1}, p^{2}, y^{1}, y^{2}\right)=Q\left(p^{1}, p^{2}, y^{1}, y^{2}\right)$ for all $\lambda>0$.

BT9: Commodity Reversal: $\quad Q\left(\Pi p^{1}, \Pi p^{2}, \Pi y^{1}, \Pi y^{2}\right)=Q\left(p^{1}, p^{2}, y^{1}, y^{2}\right)$ where $\Pi$ is an $N$ by $N$ permutation matrix.

BT10: Commensurability: $\quad Q\left(\delta_{1} p_{1}^{1}, \ldots, \delta_{N} p_{N}^{1}, \delta_{1} p_{1}^{2}, \ldots, \delta_{N} p_{N}^{2}, \delta_{1}^{-1} y_{1}^{1}, \ldots, \delta_{N}^{-1} y_{N}^{1}, \delta_{1}^{-1} y_{1}^{2}\right.$, $\left.\ldots, \delta_{N}^{-1} y_{N}^{2}\right)=Q\left(p_{1}^{1}, \ldots, p_{N}^{1}, p_{1}^{2}, \ldots, p_{N}^{2}, y_{1}^{1}, \ldots, y_{N}^{1}, y_{1}^{2}, \ldots, y_{N}^{2}\right)$ for all $\delta_{1}>0, \ldots, \delta_{N}>0$.

BT11: Time Reversal: $\quad Q\left(p^{2}, p^{1}, y^{2}, y^{1}\right)=1 / Q\left(p^{1}, p^{2}, y^{1}, y^{2}\right)$.

BT12: Monotonicity in Current Period Quantities: $\quad Q\left(p^{1}, p^{2}, y^{1}, y^{2}\right)<Q\left(p^{1}, p^{2}, y^{1}, y\right)$ if $y^{2}<y$.

BT13: Monotonicity in Base Period Quantities: $\quad Q\left(p^{1}, p^{2}, y^{1}, y^{2}\right)>Q\left(p^{1}, p^{2}, y, y^{2}\right)$ if $y^{1}<y$.

It should be noted ${ }^{34}$ that the Fisher ideal quantity index $Q_{F}$ satisfies all of the above 13 bilateral tests.

Proposition 8: Let the bilateral quantity index $Q$ satisfy tests BT1-BT13. Then the Gini multilateral system defined by (54) and (58) satisfies all of the multilateral tests except $T 10, T 11$ and $T 12$. However, the Gini system satisfies a modified version of $T 11$ where $Q_{F}$ is replaced by $Q$. If $Q$ equals the Fisher ideal quantity index $Q_{F}$, then the Gini-EKS system passes all of the multilateral tests except the consistency in aggregation test $T 10$ and the additivity test $T 12$. In addition, the Gini-EKS multilateral system is exact for the aggregator function defined by (41) and the unit cost function defined by (42).

The above Proposition shows that the Gini-EKS system has desirable properties from both the economic point view (since it is superlative) as well as from the test point of view (since it fails only two tests).

As a useful application of the first part of Proposition 8, we note that the Walsh $[1901 ; 105]$ quantity index $Q_{W}$ defined as

$$
Q_{W}\left(p^{1}, p^{2}, y^{1}, y^{2}\right) \equiv \Sigma_{n=1}^{N}\left(p_{n}^{1} p_{n}^{2}\right)^{1 / 2} y_{n}^{2} / \Sigma_{m=1}^{N}\left(p_{m}^{1} p_{m}^{2}\right)^{1 / 2} y_{m}^{1}
$$


satisfies all of the bilateral tests BT1-BT13. Hence applying Proposition 8, the Gini multilateral system defined by (54) and (58) where $Q=Q_{W}$ satisfies all of the multilateral tests except $T 10, T 11$ and $T 12$. Moreover, if we modify test $T 11$ by replacing $Q_{F}$ by $Q_{W}$, this modified test $T 11$ will be satisfied by the Gini-Walsh multilateral system. Finally, Diewert [1976; 130-134] showed that the Generalized Leontief unit cost function defined by $^{35}$

$$
c\left(p_{1}, \ldots, p_{N}\right) \equiv \Sigma_{i=1}^{N} \Sigma_{j=1}^{N} b_{i j} p_{i}^{1 / 2} p_{j}^{1 / 2}
$$

where $b_{i j}=b_{j i}$ is exact for (64). In a manner analogous to the proof of Proposition 8, we can show that the $c$ defined by (65) is exact for the system of functional equations (13) when the country shares are defined by (54) and (58) with $Q \equiv Q_{W}$. Thus the Gini multilateral methods that use either the Fisher or Walsh quantity indexes, $Q_{F}$ or $Q_{W}$, as the bilateral $Q$ in (58) have entirely similar axiomatic and economic properties; both are superlative multilateral methods.

We turn now to another superlative multilateral method with good axiomatic properties.

\section{The Own Share System}

Given a bilateral quantity index $Q$, if we pick a base country $i$, we can calculate the quantity aggregate for country $k$ relative to $i$ by $Q\left(p^{i}, p^{k}, y^{i}, y^{k}\right)$. If we sum these numbers over $k$, we obtain total bloc output or consumption relative to the base country $i$. Hence country $i$ 's share of bloc output, using country $i$ as the base, is the reciprocal of this sum, $S^{i *}$ defined as:

$$
S^{i *} \equiv\left[\Sigma_{k=1}^{K} Q\left(p^{i}, p^{k}, y^{i}, y^{k}\right)\right]^{-1}=\left[\Sigma_{k=1}^{K} Q\left(p^{k}, p^{i}, y^{k}, y^{i}\right)^{-1}\right]^{-1}
$$

where the last equality in (66) follows if $Q$ satisfies the time reversal test. Unfortunately, unless $Q$ satisfies the circularity test, the "shares" defined by (66) will not in general sum to unity. Hence, we need to normalize the $S^{i *}$ so that they sum to one. Thus the own share multilateral system is defined by (54) and the following $K$ equations:

$$
S^{i} \equiv \alpha\left[\Sigma_{k=1}^{K} Q\left(p^{k}, p^{i}, y^{k}, y^{i}\right)^{-1}\right]^{-1} ; \quad i=1, \ldots, K .
$$

The own share system was introduced by Diewert [1986] [1988; 69]. The preliminary "share" $S^{i *}$ defined by (66) defines country $i$ 's share of world product (or consumption or input) in the metric of country $i$. Since in general, these metrics are not quite compatible, these shares are adjusted to sum up to unity using (67) and (54). 
It can be shown ${ }^{36}$ that the own shares defined by (67) and (54) will be numerically close to the Gini shares defined by (58) and (54) (if the same $Q$ is used in (58) and (67)), since equations (67) can be replaced by the following equivalent system of equations:

$$
S^{i}=\alpha\left[\Sigma_{k=1}^{K}(1 / K) Q\left(p^{k}, p^{i}, y^{k}, y^{i}\right)^{-1}\right]^{-1} ; \quad i=1, \ldots, K .
$$

In (58), a geometric mean of the numbers $Q\left(p^{1}, p^{i}, y^{1}, y^{i}\right), \ldots, Q\left(p^{K}, p^{i}, y^{K}, y^{i}\right)$ is taken while in (68), a harmonic mean is taken. Since a geometric mean will usually closely approximate a harmonic mean, it is evident that the Gini shares will usually be numerically close to the own shares.

The following Proposition shows that the axiomatic and economic properties of the own share system are almost identical to the axiomatic and economic properties of the Gini system.

Proposition 9: Let the bilateral quantity index $Q$ satisfy tests BT1-BT13. Then the own share system defined by (54) and (67) fails the multilateral linear homogeneity test $T 8$ and the additivity test $T 12$. Test $T 11$ is satisfied if $Q$ equals $Q_{F}$, the Fisher ideal quantity index, and in general, a modified test $T 11$ is satisfied where the $Q_{F}$ in the statement of the test is replaced by the bilateral $Q$. All remaining multilateral tests are satisfied. If $Q$ equals $Q_{F}$, then the own share system is exact for the homogeneous quadratic aggregator function $f$ defined by (41) and for the homogeneous quadratic unit cost function $c$ defined by (42).

The above Proposition shows that the Fisher own share system (where $Q=Q_{F}$ ) is superlative and has desirable axiomatic properties. Its properties are identical to the Gini-EKS system studied in the previous section with the exception of tests $T 8$ and $T 10$ : the Fisher own share system satisfies the country partitioning test $T 10$ and fails the homogeneity in quantities test $T 8$ and vice verse for the Gini-EKS system. Both methods fail the additivity test $T 12$. Thus if the linear homogeneity property $T 8$ were thought to be more important than the country weighting property $T 10$, then the Gini-EKS system should be favored over the Fisher own share system and vice versa.

As a corollary to Proposition 9, we note that the Walsh index $Q_{W}$ defined by (64) satisfies the bilateral tests BT1-BT13. Hence the Walsh own share system (where $Q \equiv Q_{W}$ in (67)) passes all of the multilateral tests except $T 8, T 11$ and $T 12$. Moreover, a modified test $T 11$ (where $Q_{F}$ is replaced by $Q_{W}$ in the statement of the test) is satisfied. Finally, it can be shown that the generalized Leontief unit cost function defined by (65) is exact for the system of functional equations (13) where the country shares are defined by (54) and (67) with $Q \equiv Q_{W}$. Hence the Walsh own share system is also a superlative method. 


\section{Generalizations of Van Yzeren's Unweighted Balanced Method}

In this section, we consider generalizations of Van Yzeren's [1956; 25] unweighted balanced multilateral method. In the following section, we consider generalizations of his weighted balanced method.

Let $P\left(p^{j}, p^{k}, y^{j}, y^{k}\right)$ be a bilateral price index and consider the following minimization problem:

$$
\min _{P_{1}, \ldots, P_{K}} \Sigma_{j=1}^{K} \Sigma_{k=1}^{K} P\left(p^{j}, p^{k}, y^{j}, y^{k}\right) P_{j} / P_{k}
$$

Note the similarity of (69) to the minimization problem (60) which generated the Gini price levels.

Since the multilateral methods defined in this section and in section 10 are both generated by solving minimization problems, both methods are examples of what Diewert [1981; 179] called neostatistical approaches to multilateral comparisons.

The first order necessary conditions for the minimization problem (69) reduce to

$$
\Sigma_{k=1}^{K} P\left(p^{i}, p^{k}, y^{i}, y^{k}\right) P_{i} / P_{k}=\Sigma_{j=1}^{K} P\left(p^{j}, p^{i}, y^{j}, y^{i}\right) P_{j} / P_{i} ; \quad i=1, \ldots, K .
$$

Note that the objective function in (69) is homogeneous of degree zero in the $P_{1}, \ldots, P_{K}$. Thus a normalization on the $P_{k}$ can be imposed without changing the minimum. Van Yzeren $[1956 ; 25-26]^{37}$ initially defined the bilateral price index $P\left(p^{j}, p^{k}, y^{j}, y^{k}\right)$ to be the Laspeyres price index ${ }^{38}$ and proved that the minimum to (69) exists and is characterized by a unique positive solution ray to the first order conditions (70). ${ }^{39}$ Van Yzeren's proofs of existence and uniqueness go through for the more general model with a general bilateral $P$ provided that the $P\left(p^{j}, p^{k}, y^{j}, y^{k}\right)$ are all positive.

The minimization problem (69) involving the price levels $P_{k}$ can be converted into a minimization problem involving the $S_{k}$ if we use equations (59) to eliminate the $P_{k}$ in (69). If we then eliminate the $P\left(p^{j}, p^{k}, y^{j}, y^{k}\right)$ using the product test (51), the minimization problem (69) becomes:

$$
\begin{aligned}
& \min _{S_{1}, \ldots, S_{K}} \Sigma_{j=1}^{K} \Sigma_{k=1}^{K}\left[1 / Q\left(p^{j}, p^{k}, y^{j}, y^{k}\right)\right]\left[S_{k} / S_{j}\right] \\
&=\min _{S_{1}, \ldots, S_{K}} \Sigma_{j=1}^{K} \Sigma_{k=1}^{K} Q\left(p^{k}, p^{j}, y^{k}, y^{j}\right) S_{k} / S_{j}
\end{aligned}
$$

where (71) follows from the line above if $Q$ satisfies the bilateral time reversal test BT11. The first order conditions for (71) reduce to

$$
\Sigma_{j=1}^{K} Q\left(p^{i}, p^{j}, y^{i}, y^{j}\right) S_{i} / S_{j}=\Sigma_{k=1}^{K} Q\left(p^{k}, p^{i}, y^{k}, y^{i}\right) S_{k} / S_{i} ; \quad i=1, \ldots, K .
$$

As was the case with equations (70), equations (72) are dependent and any one of them can be dropped. Following Van Yzeren's [1956; 25-26] proof again and assuming that the 
$Q\left(p^{j}, p^{k}, y^{j}, y^{k}\right)$ are all positive, we obtain a unique positive solution ray to (72). To obtain a unique solution to (72), add the usual normalization

$$
\Sigma_{k=1}^{K} S_{k}=1
$$

Following the example of Van Yzeren [1956; 19], we suggest a practical method for finding the solution to (72) and (73). First, note that equations (72) can be rewritten as follows: for $i=1, \ldots, K$ :

$$
S_{i}=\left\{\left[\Sigma_{k=1}^{K} Q\left(p^{k}, p^{i}, y^{k}, y^{i}\right) S_{k}\right] /\left[\Sigma_{j=1}^{K} Q\left(p^{i}, p^{j}, y^{i}, y^{j}\right) S_{j}^{-1}\right]\right\}^{\frac{1}{2}} .
$$

Temporarily set $S_{1}=1$ and drop the first equation from (74). Insert positive starting values for $S_{2}, \ldots, S_{K}$ into the right hand sides of equations 2 to $K$ in (74) and obtain new values for $S_{2}, \ldots, S_{K}$. Insert these new values into the right hand sides of equations (74) and keep iterating until the $S_{i}$ converge. The final vector $\left[1, S_{2}^{*}, \ldots, S_{K}^{*}\right]$ can be then be normalized to sum up to unity. ${ }^{40}$

Before we discuss the axiomatic properties of the multilateral method defined by (72) and (73), it is useful to note what happens if the circularity test (52) is satisfied by $Q$ for the observed data set. At the beginning of section 10, we showed that all of the star system shares would coincide in this case. If the common system of shares were denoted by $S_{1}^{*}, \ldots, S_{K}^{*}$, we would have $Q\left(p^{i}, p^{j}, y^{i}, y^{j}\right)=S_{j}^{*} / S_{i}^{*}$ for all $i$ and $j$. Thus if the bilateral index $Q$ satisfies circularity for the observed data, then it can be seen that the base country invariant shares $S_{1}^{*}, \ldots, S_{K}^{*}$ will satisfy equations (72) and hence these shares will also be the unweighted balanced method shares. ${ }^{41}$

Proposition 10: Let the once differentiable bilateral quantity index $Q$ satisfy tests BT1BT13. Then the unweighted Van Yzeren balanced system with this $Q$ defined by (72) and (73) fails the multilateral tests $T 10$ and $T 12$. Test $T 11$ is satisfied if $Q$ equals $Q_{F}$, the Fisher ideal quantity index, and in general, a modified test $T 11$ is satisfied where the $Q_{F}$ in the statement of the test is replaced by $Q$. The remaining multilateral tests are satisfied. If $Q$ equals the Laspeyres, Paasche or Fisher ideal quantity index, then the corresponding unweighted balanced system is exact for the homogeneous quadratic aggregator function $f$ defined by (41) and for the homogeneous quadratic unit cost function $c$ defined by (42). Moreover, each of these three versions of the unweighted balanced method satisfies all of the multilateral tests except the country partitioning test $T 10$ and the additivity test $T 12$.

The above Proposition shows that the following multilateral methods are all superlar tive: (i) Van Yzeren's [1956; 15-20] original unweighted balanced method that set $Q=Q_{L}$, where $Q_{L}$ is the Laspeyres quantity index (which corresponds via (51) to the Paasche price index); (ii) Gerardi's [1974] modified unweighted balanced method that set $Q=Q_{P}$, where 
$Q_{P}$ is the Paasche quantity index (which corresponds to the Laspeyres price index) and (iii) Van Ijzeren's $[1987 ; 61]$ Fisher ideal balanced method that set $Q=Q_{F}$, where $Q_{F}$ is the Fisher ideal quantity index (which corresponds via (51) to the Fisher ideal price index). Moreover, these three methods all have the same axiomatic properties, failing only tests $T 10$ and $T 12$.

Since the Walsh bilateral quantity index $Q_{W}$ satisfies tests BT1-BT13, Proposition 10 shows that the unweighted balanced method that sets $Q=Q_{W}$ in (72) also satisfies all of the multilateral tests except $T 10, T 11$ and $T 12$. However, the modified version of $T 11$ where $Q_{F}$ is replaced by $Q_{W}$ is satisfied. Moreover, it is straightforward to show that this Walsh unweighted balanced method is exact for the flexible unit cost function defined by (65) and hence this multilateral method is also superlative.

It is possible to follow the example of Balk $[1989 ; 310-311]$ and show that the shares generated by the unweighted balanced method with an arbitrary bilateral $Q$ (recall (72) above) will be numerically close to the shares generated by the Gini system (recall (58) above). First note that if we multiply both sides of (72) by $1 / K$, we obtain arithmetic means of $K$ numbers on each side of (72). These arithmetic means can usually be closely approximated by geometric means. Hence the equations (72) are approximately equivalent to

$$
\Pi_{j=1}^{K}\left[Q\left(p^{i}, p^{j}, y^{i}, y^{j}\right) S_{i} / S_{j}\right]^{1 / K}=\Pi_{k=1}^{K}\left[Q\left(p^{k}, p^{i}, y^{k}, y^{i}\right) S_{k} / S_{i}\right]^{1 / K} ; \quad i=1, \ldots, K .
$$

The above equations simplify to

$$
S_{i}^{2}=\alpha^{2}\left[\Pi_{k=1}^{K} Q\left(p^{k}, p^{i}, y^{k}, y^{i}\right) / \Pi_{j=1}^{K} Q\left(p^{i}, p^{j}, y^{i}, y^{j}\right)\right]^{1 / K} ; \quad i=1, \ldots, K,
$$

where $\alpha \equiv\left[\Pi_{k=1}^{K} S_{k}\right]^{1 / K}$. If $Q$ satisfies the time reversal test BT11, then equations (76) further simplify to equations (58), the defining equations for the Gini shares with a general $Q$. Finally, note that if the bilateral $Q$ in (76) is either the Laspeyres index $Q_{L}$ or the Parsche index $Q_{P}$, then the resulting equations (76) are equivalent to equations (58) with the bilateral $Q$ in (58) set equal to the Fisher ideal quantity index $Q_{F}$. This last observation helps to explain Van Ijzeren's $[1987 ; 63]$ observation that the unweighted balanced method shares are numerically close no matter whether $Q_{L}, Q_{P}$ or $Q_{F}$ is used as the bilateral $Q$ in equations (72) and (76). ${ }^{42}$

The argument in the last paragraph above showed that the Fisher unweighted balanced method, where $Q=Q_{F}$ in (72), will generate shares that are numerically close to the Gini-EKS shares, where $Q=Q_{F}$ in (58). Propositions 8 and 10 above also show that these two multilateral methods have identical axiomatic properties (they both fail the country partitioning test $T 10$ and the additivity test $T 12$ ) and they have identical economic properties (they are both exact for the homogeneous quadratic functional forms defined by (41) and (42) above). 
In the following section, we shall study another class of multilateral methods due originally to Van Ijzeren [1982; 45]. The method studied in section 13 below turns out to have identical axiomatic and economic properties as the own share system studied in section 11 above.

\section{Generalizations of Van Yzeren's Weighted Balanced Method}

Following Van Yzeren $[1956 ; 25]$ (who chose the bilateral $Q$ to be $Q_{L}$, the Laspeyres quantity index), we introduce the following weighted version of the minimization problem (71):

$$
\min _{S_{1}, \ldots, S_{K}} \Sigma_{j=1}^{K} \Sigma_{k=1}^{K} w_{j} w_{k} Q\left(p^{k}, p^{j}, y^{k}, y^{j}\right) S_{k} / S_{j}
$$

where the positive weights $w_{j}$ are given numbers that somehow reflect the relative size or importance of the countries. The first order necessary conditions for the above minimization problem reduce to (77) for $i=1, \ldots, K$ :

$$
\Sigma_{j=1}^{K} w_{j} Q\left(p^{i}, p^{j}, y^{i}, y^{j}\right) S_{i} / S_{j}=\Sigma_{k=1}^{K} w_{k} Q\left(p^{k}, p^{i}, y^{k}, y^{i}\right) S_{k} / S_{i}
$$

If the bilateral $Q$ satisfies BT1 and we add the normalization (73) to (77), then the arguments of Van Yzeren [1956; 25-26] can be adapted to show that there is a unique positive set of shares $S_{1}(P, Y, w), \ldots, S_{K}(P, Y, w)$ that solve (73) and (77). Note that the solution shares now depend on the vector of country weights $w \equiv\left(w_{1}, \ldots, w_{K}\right)^{T}$ as well as the matrix of country prices $P$ and the matrix of country quantities $Y$. At this point, we note that Balk's [1989][1995] axiomatic treatment of multilateral index numbers works with this weighted system of share functions, $S_{1}(P, Y, w), \ldots, S_{K}(P, Y, w)$, rather than the unweighted shares, $S_{1}(P, Y), \ldots, S_{K}(P, Y)$, that have been studied in the present paper. We will not pursue Balk's axiomatic treatment, since it adds an extra layer of complication in determining exactly what weights $w$ should be used. Moreover, our axiomatic treatment of the multilateral case seems to be the simplest extension of the bilateral axiomatic approach.

We now follow Van Ijzeren $[1983 ; 45][1987 ; 65]$ and set $w_{j}=S_{j}$ for $j=1, \ldots, K$ in (77)..$^{43}$ This leads to the following system of equations:

$$
\Sigma_{j=1}^{K} Q\left(p^{i}, p^{j}, y^{i}, y^{j}\right) S_{i}^{2}=\Sigma_{k=1}^{K} Q\left(p^{k}, p^{i}, y^{k}, y^{i}\right) S_{k}^{2} ; \quad i=1, \ldots, K .
$$

Equations (78) and the normalizing equation (73) define the Van Ijzeren weighted balanced shares with a general bilateral $Q$. Summing equations (78) over all $i$ leads to an identity so only $K-1$ of the $K$ equations in (78) are independent. 
We need to establish the existence of a positive unique solution to equations (73) and $(78)^{44}$ define the $i k$ th element of the matrix $A$ by

$$
a_{i k} \equiv Q\left(p^{k}, p^{i}, y^{k}, y^{i}\right) / \Sigma_{j=1}^{K} Q\left(p^{i}, p^{j}, y^{i}, y^{j}\right) ; \quad 1 \leq i, k \leq K
$$

It can be seen that equations (78) are equivalent to the following system of equations where $x^{T} \equiv\left[x_{1}, \ldots, x_{K}\right] \equiv\left[S_{1}^{2}, \ldots, S_{K}^{2}\right]$ :

$$
A x=x
$$

We assume $Q$ satisfies BT1 and hence $A$ has positive elements. Define the vector $v \equiv$ $\left[v_{1}, \ldots, v_{K}\right]^{T}$ where $v_{i} \equiv \Sigma_{j=1}^{K} Q\left(p^{i}, p^{j}, y^{i}, y^{j}\right)$ for $i=1, \ldots, K$. Using this definition for $v$ and $(79)$, we have

(81) $v^{T}=v^{T} A$.

Equation (81) shows that the positive vector $v$ is a left eigenvector of the positive matrix $A$ that corresponds to a unit eigenvalue. Hence by the Perron [1907] - Frobenius [1909] Theorem, the maximal positive eigenvalue of $A$ is one and there exists a corresponding strictly positive right eigenvector $x$ that satisfies (80). Once $x$ is determined, the corresponding $S_{i}$ satisfying (73) and (78) can be defined by

$$
S_{i}=x_{i}^{1 / 2} / \Sigma_{j=1}^{K} x_{j}^{1 / 2} ; \quad i=1, \ldots, K
$$

The numerical calculation of the weighted balanced shares can readily be accomplished if we make use of the theory of positive matrices. Let us drop the last equation in equations (80) and set the last component of the $x$ vector equal to 1 . Define the top left $K-1$ by $K-1$ block of the $K$ by $K$ matrix $A$ as the positive matrix $\tilde{A}$, define the first $K-1$ components of the $K$ dimensional column vector $x$ as $\tilde{x}$ and define the top right $K-1$ by 1 block of $A$ as the positive vector $\tilde{a}$. Setting $x_{K}=1$, the first $K-1$ equations in (80) may be rewritten as

$$
\tilde{x}=\left[I_{K-1}-\tilde{A}\right]^{-1} \tilde{a}
$$

where $I_{K-1}$ is a $K-1$ by $K-1$ identity matrix. Using a result due to Frobenius [1908; 473], the maximal positive eigenvalue of $\tilde{A}$ is strictly less than the maximal positive eigenvalue of $A$, which is 1 . Thus the inverse of $I_{K-1}-\bar{A}$ has the following convergent matrix power series representation:

$$
\left[I_{K-1}-\tilde{A}\right]^{-1}=I_{K-1}+\tilde{A}+\tilde{A}^{2}+\ldots
$$


and hence, using the positivity of $\tilde{A},\left[I_{K-1}-\tilde{A}\right]^{-1}$ is a matrix with strictly positive elements. Thus, using the positivity of $\tilde{a}$, the $\tilde{x}$ defined by (83) has positive components. Equations (83), $x_{N}=1$ and equations (82) can be used to numerically define the weighted balanced shares $S_{i}$ using a general bilateral $Q$ satisfying BT1. ${ }^{45}$

The following proposition lists the axiomatic and economic properties of the multilateral method defined by (73) and (78).

Proposition 11: Let the once differentiable bilateral quantity index $Q$ satisfy tests BT1BT13. Then the weighted balanced method with the general bilateral $Q$ defined by (73) and (78) fails the multilateral homogeneity in quantities test $T 8$ and the additivity test T12. Test $T 11$ is satisfied if $Q=Q_{F}$, the Fisher ideal quantity index, and in general, a modified test $T 11$ is satisfied where the $Q_{F}$ in the statement of the test is replaced by the $Q$ satisfying the bilateral tests BT1-BT13. The remaining multilateral tests are satisfied. If the bilateral quantity index $Q$ in (78) equals the Laspeyres, Paasche or Fisher ideal quantity index, then the resulting Van Ijzeren $[1983 ; 45]$ weighted balanced systems are exact for the homogeneous quadratic functions $f$ and $c$ defined by (41) and (42) and hence each of these systems is superlative. Moreover, each of these three versions of the weighted balanced method satisfies all of the multilateral tests except $T 8$ and $T 12$.

The above Proposition shows that the Van Ijzeren [1983; 45-46] weighted balanced methods that used the Laspeyres, Paasche and Fisher quantity indexes as the bilateral quantity index are all superlative multilateral systems; i.e., they are exact for the flexible functional forms defined by (41) and (42). Moreover, these three weighted balanced methods all have excellent axiomatic properties, failing only tests $T 8$ and $T 12$.

Since the Walsh bilateral quantity index satisfies tests BT1-BT13, Proposition $11 \mathrm{im}$ plies that the weighted balanced method that uses $Q_{W}$ in (78) will satisfy all of the multilateral tests except $T 8, T 11$ and $T 12$. Moreover, this Walsh weighted balanced method will satisfy the modified version of test $T 11$ where $Q_{F}$ is replaced by $Q_{W}$. It can be shown that this method is exact for the flexible unit cost function defined by (65) and hence the Walsh weighted balanced method is also superlative.

Adapting the method used by Balk $[1989 ; 310-311]$, it is possible to show that the shares generated by the weighted balanced method using the bilateral quantity index $Q$ (see equations (78) above) will usually be numerically close to the shares generated by the Gini system using the same bilateral $Q$ (see equations (58) above). Multiply both sides of equations (78) by $1 / K$ and note that we have an arithmetic mean of $K$ numbers on each side of each equation in (78). Approximating these arithmetic means by geometric means leads to the following system of equations:

$$
\Pi_{j=1}^{K}\left[Q\left(p^{i}, p^{j}, y^{i}, y^{j}\right) S_{i}^{2}\right]^{1 / K}=\Pi_{k=1}^{K}\left[Q\left(p^{k}, p^{i}, y^{k}, y^{i}\right) S_{k}^{2}\right]^{1 / K} ; \quad i=1, \ldots, K .
$$


Equations (85) simplify to equations (76) and if $Q$ satisfies the time reversal test, equations (76) further simplify to equations (58), the defining equations for the Gini system shares. Thus if the arithmetic means are close to the corresponding geometric means in (85), the Gini shares using a bilateral $Q$ that satisfies BT11 will be close to the corresponding weighted balanced shares using the same bilateral $Q$.

Recall that if the geometric means in (75) are close to the corresponding arithmetic means, then the Gini shares using a $Q$ that satisfies BT11 will be close to the corresponding unweighted balanced shares using the same bilateral $Q$. Finally, recall that if the harmonic means in (68) are close to the corresponding geometric means, then the own shares using $Q$ will be close to the corresponding Gini shares using the same $Q$. Under normal conditions, these arithmetic, geometric and harmonic means will closely approximate each other and so the Gini shares, own shares, unweighted balanced shares and weighted balanced shares using the same bilateral $Q$ should closely approximate each other.

In sections 10 and 12, we showed that the Gini-EKS system and the unweighted balanced method with $Q=Q_{F}$ had identical axiomatic (both failed tests $T 10$ and $T 12$ ) and economic properties (both were exact for the same flexible functional forms defined by (41) and (42) above). Propositions 9 and 11 show that the own share system with $Q=Q_{F}$ and the weighted balanced system with $Q=Q_{F}$ have identical axiomatic properties (both fail tests $T 8$ and $T 12$ ) and economic properties (both are exact for the flexible functional forms defined by (41) and (42) above).

\section{What are the Tradeoffs?}

We have considered in some detail the axiomatic and economic properties of 10 methods for making multilateral comparisons. ${ }^{46}$ From the axiomatic perspective we find that the methods described in section 4, 6, 8 and 9 are dominated by other methods. The undominated methods are: (i) the Gerardi-Walsh geometric average price method defined in section 5 by equations (3) and (16) which fails only $T 10$ and $T 11$; (ii) the Geary-Khamis method defined in section 7 which fails only $T 8, T 9$ and $T 11$ (but satisfies a modified version of $T 11$ ); (iii) the Gini system defined in section 10 which fails only $T 10$ and $T 12$; (iv) the unweighted balanced system defined in section 12 which also fails only $T 10$ and $T 12$; (v) the own share system defined in section 11 which fails only $T 8$ and $T 12$ and (vi) the weighted balanced system defined in section 13 which also fails only $T 8$ and $T 12$.

From the economic perspective, we found that the four methods described in sections 10-13 were superior to the remaining six methods: the Gini-EKS system, the weighted and unweighted balanced systems with the bilateral quantity index $Q$ chosen to be the Fisher ideal index $Q_{F}$ and the own share system with $Q=Q_{F}$ were all superlative methods; i.e., they were exact for the flexible functional forms defined by (41) and (42). The other six 
methods were either not exact for any aggregator function or they were consistent only for preference functions or production functions that exhibited either perfect substitutability (a linear aggregator function) or zero substitutability (a Leontief aggregator function or a linear unit cost function).

Examining the four superlative methods defined in sections 10-13, we found that if various harmonic and arithmetic means are close to the corresponding geometric means, the shares for these four methods will be numerically close to each other if the same bilateral $Q$ is used in each method. Assuming that the bilateral quantity index used in each of these four methods is the Fisher ideal quantity index, Propositions 8-11 above showed that it was not possible for any of these superlative methods to simultaneously satisfy test $T 8$ (linear homogeneity in quantities) and test $T 10$ (country partitioning): the Gini-EKS system and the unweighted balanced method satisfied $T 8$ but not $T 10$ while the own share and the weighted balanced methods satisfied $T 10$ but not $T 8{ }^{47}$

How should we resolve the conflict between $T 8$ and $T 10$ ? There is no completely scientific answer to this question but consider the following opinions. First, Peter Hill noted that a major advantage of the Geary-Khamis method which satisfies $T 10$ over the Walsh-Gerardi method which does not is that the former method would not change very much if a large country were split up into several small countries:

"Thus, the contribution of the United States to the determination of the average international price would tend to be the same whether or not the United States were treated as a single country or fifty or more separate states."

$$
\text { Peter Hill[1982; 50] }
$$

In a similar vein, Kravis, Summers and Heston make the following comment on the WalshGerardi method:

"The Gerardi method would assign the same weight to Luxembourg and Belgium prices as to German and Netherlands prices in a comparison involving the four countries. However, if Belgium and Luxembourg become one country their average prices would have a combined weight of one. The comparison between Germany and Netherlands would differ according to whether Luxembourg and Belgium were treated as two countries or one."

Irving Kravis, Robert Summers and Alan Heston[1982; 408]

Finally, Van Ijzeren summarizes his discussion on whether a weighted method (which satisfies test T10) should be used as follows:

"Hence, theory rejects non-weighting. Surely, common sense does too!"

$$
\text { J. Van Ijzeren[1987; 67] }
$$


I tend to agree with the above authors on the importance of weighting: it seems reasonable that the chosen multilateral method should reflect the fact that if big countries are broken up into a bunch of smaller countries, then comparisons between the unpartitioned countries should remain the same. This is the essence of the country partitioning test T10. Thus it seems to me to be more important to satisfy $T 10$ rather than $T 8$.

Propositions 9 and 11 show that the Fisher own share system and the Fisher weighted balanced method have identical axiomatic and economic properties: both are superlative and both fail the linear homogeneity test $T 8$ and the additivity test $T 12$ but pass the other tests including $T 10$. Moreover, we have provided theoretical arguments to show that they will normally closely approximate each other numerically. ${ }^{48}$ Which of these two methods should be used in practice? Balk $[1989 ; 310]$ provides a theoretical argument (which the present author finds unconvincing) for preferring the weighted balanced method over the own share method. However, a major advantage of the own share method is its relative simplicity. Statistical Agencies can readily explain the essence of the method to the public as follows: each country's preliminary share of "world" output or consumption is determined by making bilateral index number comparisons (using the best available index number formula) with all other countries. These preliminary shares are then scaled (if necessary) to sum up to one. It is very difficult to explain the mechanics of the weighted balanced method in an equally simple fashion.

In some situations, it may not be important for the multilateral method to satisfy the country partitioning test $T 10$. For example, the multilateral method might be required to determine the relative price levels (or purchasing power parities) in a number of cities where an international organization or multinational firm has employees so that salaries can be set in an equitable manner. In this case, it will probably be more important to satisfy the linear homogeneity test $T 8$ rather than $T 10$. In this situation, it will be important to use a superlative method, which will recognize the realities of consumer substitution. In this situation, we would recommend the use of the Gini-EKS system or the unweighted balanced method with $Q=Q_{F}$ since these methods are superlative and fail only tests $T 10$ and $T 12$. In section 12 , we indicated that these two methods will normally numerically approximate each other quite closely. ${ }^{49}$ Which of these two methods should be used in empirical applications? On grounds of simplicity, we would favor the Gini-EKS system over the unweighted balanced system. In the former case, there is at least a closed form formula for the country shares, while in the latter case, iterative methods have to be used in order to determine the country shares. Thus it will be more difficult for International Agencies or multinational firms to explain the mechanics of the unweighted balanced method to their employees.

Having discussed the tradeoffs between tests $T 8$ and $T 10$ in the context of the four superlative multilateral methods analyzed in this paper, we turn now to a discussion of the 
tradeoffs between superlativeness and additivity. For the ten multilateral methods studied in this paper, it is impossible to satisfy both properties simultaneously if the number of countries $K$ exceeds two. ${ }^{50}$ We will now indicate why the quest for an additive superlative method will be futile in general in the many country case (i.e., when $K \geq 3$ ).

Consider the two good, three country case. Suppose that we are in the consumer context and that the preferences of each country over combinations of the two goods can be represented by the same utility function and that the observed consumption vector $\left(y_{1}^{k}, y_{2}^{k}\right)$ for each country $k$ is on the same indifference curve. Suppose further that relative prices $p_{2}^{k} / p_{1}^{k}$ differ dramatically across the three countries. The situation is depicted in Figure 1 below. The points $A, B$ and $C$ represent the consumption vectors $\left(y_{1}^{1}, y_{2}^{1}\right),\left(y_{1}^{2}, y_{2}^{2}\right)$ and $\left(y_{1}^{3}, y_{2}^{3}\right)$ for countries 1,2 and 3 respectively. Since the consumption vectors are all on the same indifference curve, a multilateral method based on the economic approach should make the country shares of world consumption equal; i.e., an economic based multilateral

\section{Figure 1}

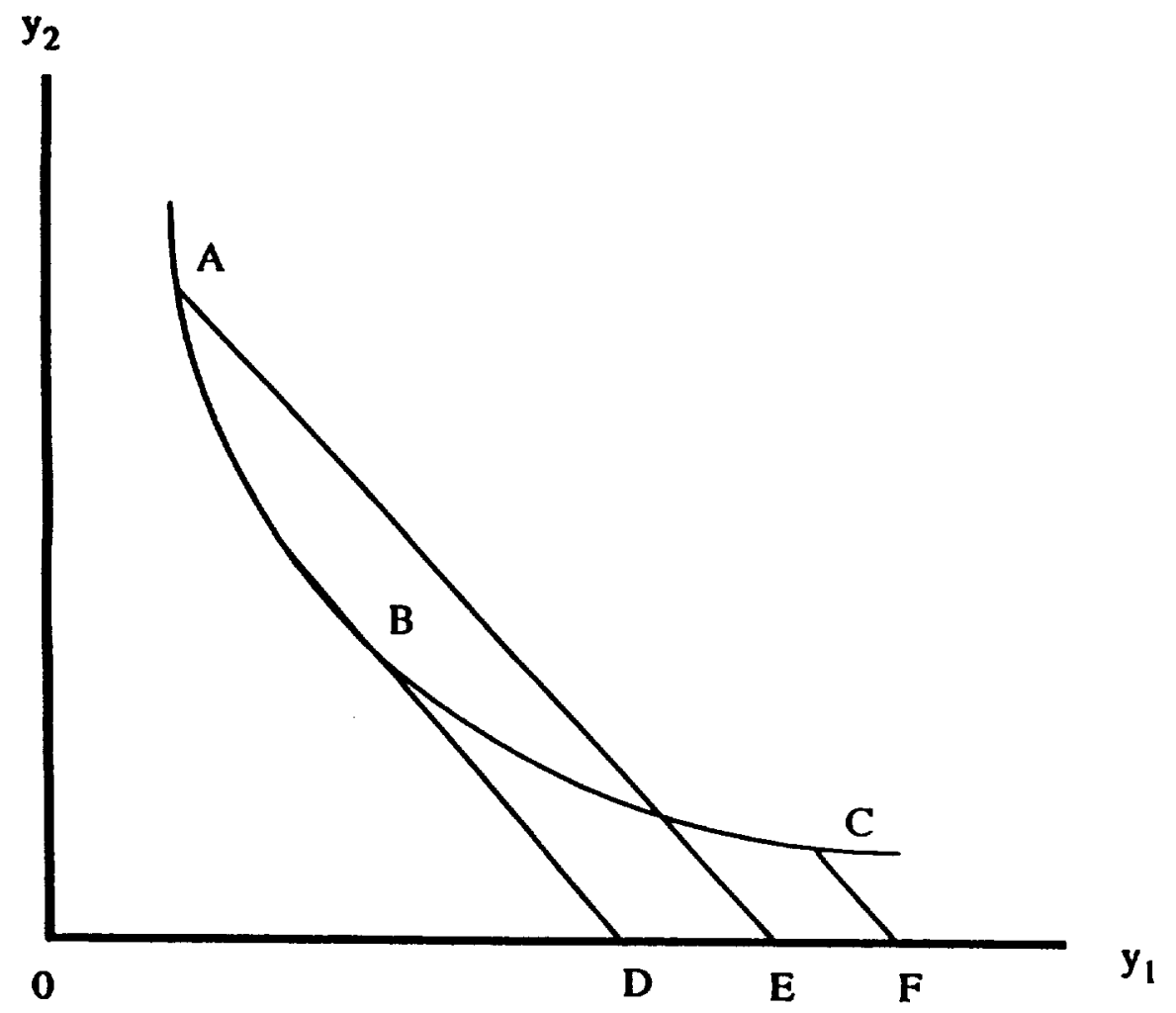

method should yield $S^{1}=S^{2}=S^{3}$. Depending on how well the flexible functional form associated with a superlative multilateral method approximates the indifference curve in Figure 1, a superlative multilateral method should lead to approximately equal shares for the three countries. The set of consumption vectors that an additive method will regard 
as being equal can be represented as a straight line with a negative slope in Figure 1. If we take the prices of country 2 as the world average prices associated with an additive multilateral method, it can be seen that the share of country 1 will be proportional to the distance OE, the share of country 2 will be proportional to OD (too small) and the share of country 3 will be proportional to OF (too big). As the reader can see, there is no choice of price weights that will generate a straight line that will pass through each of the points $A, B$ and $C$ simultaneously. Thus additive methods, which implicitly assume that indifference curves are linear, are inherently biased if indifference curves are nonlinear.

Figure 1 can also be used to demonstrate the general impossibility of finding an additive superlative multilateral method if the number of countries $K \geq 3$ and the number of commodities $N \geq 2$ : if $N>2$ and $K \geq 3$, then let the last $N-2$ components of the country consumption vectors $y^{1}, y^{2}, \ldots, y^{K}$ be identical and let the first two components of $y^{1}, y^{2}$ and $y^{3}$ be the points $A, B$ and $C$ in Figure 1, so that the utility of $y^{1}, y^{2}$ and $y^{3}$ is identical. Since there is still no straight line that will pass through the points $A, B$ and $C$, our general impossibility result follows.

Figure 1 also illustrates the Gerschenkron effect: in the consumer theory context, countries whose price vectors are far from the "international" or world average prices used in an additive method will have quantity shares that are biased upwards. ${ }^{51}$ Marris [1984; 52] has a diagram similar to our Figure 1 to illustrate the bias associated with additive methods in the consumer theory context. It can be seen that these biases are simply quantity index counterparts to the usual substitution biases encountered in the theory of the consumer price index. ${ }^{52}$ However, the biases in the multilateral context will usually be much larger than in the intertemporal context, since relative prices and quantities will be much more variable in the former context.

As an aside, R.J. Hill [1995; 73] noted that the average basket methods studied in section 6 above will suffer from a reverse Gerschenkron bias: in the consumer theory context, countries whose quantity vectors are far from the average basket quantities will have quantity shares $S^{k}$ that are biased downwards and this bias is reversed in the producer theory context.

The bottom line on the above discussion is that the quest for an additive multilateral method with good economic properties (i.e., a lack of substitution bias) is a doomed venture: nonlinear preferences and production functions cannot be adequately approximated by linear functions. Put another way, if technology and preference functions were always linear, there would be no index number problem and hundreds of papers and monographs on the subject would be superfluous! Thus from the viewpoint of the economic approach to index number theory (which assumes optimizing behavior on the part of economic agents), it is not reasonable to ask that the multilateral method satisfy the additivity test, $T 12$. 
We conclude this section by reinterpreting the quest for additivity. Suppose that we want an additive method, not to provide accurate economic relative shares for $K$ countries in a bloc, but simply to value the country quantity vectors $y^{1}, \ldots, y^{K}$ at a common set of "representative" prices $\pi \equiv\left[\pi_{1}, \pi_{2}, \ldots, \pi_{N}\right]^{T}$. The question is, how should we choose these "representative" or "reasonable" international prices? There appear to be two main alternatives: one due to Balk $[1989 ; 299]$ and one due to Hill $[1982 ; 59]$.

Suppose that in defining the international price vector $\pi$, we are allowed to use the country shares $S^{k}$ and country purchasing power parities or price levels $P^{k}, k=1, \ldots, K$, that are generated by the investigator's "best" multilateral method. Balk [1989; 299], drawing on the work of Van Ijzeren [1983][1987], defined his vector of international prices $\pi$ as the following country share weighted average of the country price vectors $p^{k}$ deflated by their purchasing power parities:

$$
\pi \equiv \Sigma_{k=1}^{K} S^{k}\left(p^{k} / P^{k}\right)
$$

On the other hand, the generalized Hill $[1982 ; 59]$ international prices $\pi_{n}, n=1, \ldots, N$ can be defined by equations (25) above, except that the Geary-Khamis price levels $P^{k}$ which appeared in those equations should be replaced by the analyst's "best" multilateral price levels. Hill $[1982 ; 50][1984 ; 129]$ explained why the $\pi_{n}$ 's defined by $(25)$ are natural ones to use to define international average prices: these prices are the natural extension to the multilateral context of the prices used in the national accounts of a single country. In a single country, the average price used for a commodity is its unit value; i.e., its total value divided by its total quantity. ${ }^{53}$ It can be seen that the $\pi_{n}$ defined by (25) are precisely of this character, except that the country prices $p_{n}^{k}$ are replaced by the purchasing power parity adjusted prices $p_{n}^{k} / P^{k}$. However, Hill did not emphasize the fact that it is not necessary to use the Geary-Khamis $P^{k}$ in (25): the $P^{k}$ generated by any multilateral method could be used.

To summarize the above discussion, we followed the example of Balk $[1989 ; 310]$ and suggested that it is not necessary that the multilateral method satisfy the additivity test: the country shares $S^{k}$ and the country price levels $P^{k}$ generated by the "best" multilateral method can be used in equations (25) or (86) to generate "representative" international prices or unit values $\pi_{n}$ that can be used by analysts in applications where it is important that commodity flows across countries in the bloc be valued at constant prices. ${ }^{54}$

\section{Conclusion}

In section 2, we developed a "new" 55 system of axioms or desirable properties for multilateral index numbers. Tests $T 1-T 9$ are adaptations of bilateral index number 
tests to the multilateral context. Tests $T 10$ and $T 11$ are genuine multilateral properties that do not have bilateral counterparts. We have included the additivity test $T 12$ in our list of axioms because so many analysts find this property very useful in empirical applications. However, in the previous section, we concluded that the additivity test was not at all desirable from the viewpoint of the economic approach to index number theory since additive methods cannot deal adequately with nonlinear preference and technology functions. Thus axioms $T 1-T 11$ are a very reasonable set of properties that can be used to assess the usefulness of a multilateral system of index numbers.

In section 3, we pursued the economic approach to index number comparisons. In particular, we adapted the exact and superlative index number methodology developed for bilateral index numbers to the multilateral context. If a multilateral system is superlative, then it is consistent with optimizing behavior on the part of economic agents where the common preference or technology function can provide a second order approximation to an arbitrary differentiable linearly homogeneous function. Thus a superlative method will tend to minimize various substitution biases that nonsuperlative methods will possess. Superlativeness is a minimal property from the viewpoint of the economic approach to index numbers that a multilateral system should possess.

In section 4-13, we evaluated 10 leading multilateral methods from the economic and axiomatic perspectives. From the axiomatic perspective, 6 methods satisfied more axioms than the remaining methods. These best methods were: the Gerardi-Walsh geometric average price method defined in section 5 (which fails $T 10$ and $T 11$ ); the Geary-Khamis method defined in section 7 (which fails $T 8, T 9$ and T11); the Gini system and the unweighted balanced system defined in sections 10 and 12 (which fail T10 and T12) and the own share and weighted balanced system defined in sections 11 and 13 (which fail $T 8$ and T12). From the economic perspective, the 4 methods defined in sections 10-13 were the best.

To see that the 10 multilateral methods studied in this paper can generate a very wide range of outcomes, the reader should view the results of a 3 country, 2 commodity artificial empirical example in Appendix 2.

If the multilateral method is required to determine purchasing power parities in the $K$ locations so that satisfaction of the country partitioning test $T 10$ is not important in this context, then in section 14, we concluded that either the Gini-EKS system or the unweighted balanced method (using the bilateral Fisher ideal quantity index) were probably best for this purpose. Between these two methods, we have a slight preference for the Gini-EKS method due to its relative simplicity.

On the other hand, if the multilateral method is required to rank the relative outputs or real consumption expenditures between the $K$ countries (or provinces or states), then since satisfaction of test $T 10$ is important in this context, we concluded in section 14 that 
the own share or the weighted balanced methods (using the bilateral Fisher ideal quantity index) were probably best for this purpose. Between these two methods, we have a slight preference for the own share system due to its relative simplicity. ${ }^{56}$

Finally, it is appropriate to end this paper by noting the pioneering contributions of Van Yzeren [1957] (Van Ijzeren [1983][1987]): of the 10 methods studied in this paper, he was the originator of 4 of them. 


\section{Appendix 1: Proofs of Propositions}

In most cases, verifying whether a multilateral method satisfies a given test is a straightforward calculation. Hence many proofs will be omitted.

Proof of Proposition 1:

T9. To verify monotonicity, differentiate $S^{k}$ with respect to the components of $y^{k}$ :

$\nabla_{y^{k}} S^{k}(P, Y)=\left[\Sigma_{i=1}^{K} p^{i} \cdot y^{i}\right]^{-1}\left[1-S^{k}(P, Y)\right] p^{k}>>0_{N}$

since $p^{k}>>0_{N}$ and $0<S^{k}(P, Y)<1$.

T11: Under the conditions of $T 11$, we find that

$\Sigma_{j \in B} S^{j}(P, Y) / \Sigma_{i \in A} S^{i}(P, Y)=\Sigma_{j \in B} \gamma_{j} \delta_{j} p^{b} \cdot y^{b} / \Sigma_{i \in A} \alpha_{i} \beta_{i} p^{a} \cdot y^{a} \neq Q_{F}\left(p^{a}, p^{b}, y^{a}, y^{b}\right)$

Exactness Properties: The system of functional equations (12) becomes:

$$
\begin{aligned}
& e_{i} \nabla f\left(y^{i}\right) \cdot y^{i} / e_{j} \nabla f\left(y^{j}\right) \cdot y^{j}=f\left(y^{i}\right) / f\left(y^{j}\right) \\
& \text { or } \quad e_{i} f\left(y^{i}\right) / e_{j} f\left(y^{j}\right)=f\left(y^{i}\right) / f\left(y^{j}\right) \quad \text { since } \nabla f\left(y^{i}\right) \cdot y^{i}=f\left(y^{i}\right) \\
& \text { or } \quad e_{i} / e_{j}=1 \text {. }
\end{aligned}
$$

Hence there is no differentiable linearly homogeneous $f$ which satisfies (12).

The system of functional equations (13) becomes:

$$
\begin{aligned}
& p^{i} \cdot \nabla c\left(p^{i}\right) u_{i} / p^{j} \cdot \nabla c\left(p^{j}\right) u_{j}=u_{i} / u_{j} \\
& \text { or } \quad c\left(p^{i}\right) u_{i} / c\left(p^{j}\right) u_{j}=u_{i} / u_{j} \quad \text { since } \quad p^{i} \cdot \nabla c\left(p^{i}\right)=c\left(p^{i}\right) \\
& \text { or } e_{i} / e_{j}=1 \text { since } c\left(p^{i}\right)=e_{i} \text {. }
\end{aligned}
$$

Hence there is no differentiable unit cost function $c$ that satisfies (13).

Proof of Proposition 2:

T3: Let $p>>0_{N}, \alpha_{k}>0$ and $p^{k}=\alpha_{k} p$ for $k=1, \ldots, K$. Then for $k=1, \ldots, K$ :

$$
\begin{aligned}
S^{k}(P, Y)= & \Sigma_{n=1}^{N} m\left(\alpha_{1} p_{n}, \ldots, \alpha_{K} p_{n}\right) y_{n}^{k} / \Sigma_{j=1}^{K} m\left(\alpha_{1} p_{j}, \ldots, \alpha_{K} p_{j}\right) \Sigma_{i=1}^{K} y_{j}^{i} \\
= & \Sigma_{n=1}^{N} m\left(\alpha_{1}, \ldots, \alpha_{K}\right) p_{n} y_{n}^{k} / \Sigma_{j=1}^{K} m\left(\alpha_{1}, \ldots, \alpha_{K}\right) p_{j} \Sigma_{i=1}^{K} y_{j}^{i} \\
& \quad \text { using the linear homogeneity of } m \\
= & p \cdot y^{k} / \Sigma_{i=1}^{K} p \cdot y^{i} .
\end{aligned}
$$


T7: Let $\alpha_{k}>0$ for $k=1, \ldots, K$. Then

$$
\begin{aligned}
S^{k}\left(\alpha_{1} p^{1}, \ldots, \alpha_{K} p^{K}, Y\right) & \\
& =\Sigma_{n=1}^{N} m\left(\alpha_{1} p_{n}^{1}, \ldots, \alpha_{K} p_{n}^{K}\right) y_{n}^{k} / \Sigma_{j=1}^{N} m\left(\alpha_{1} p_{j}^{1}, \ldots, \alpha_{K} p_{j}^{K}\right) \Sigma_{i=1}^{K} y_{j}^{i} \\
& \neq \Sigma_{n=1}^{N} m\left(p_{n}^{1}, \ldots, p_{n}^{K}\right) y_{n}^{k} / \Sigma_{j=1}^{N} m\left(p_{j}^{1}, \ldots, p_{j}^{K}\right) \Sigma_{i=1}^{K} y_{j}^{i}
\end{aligned}
$$

unless

$$
m\left(\alpha_{1} p_{n}^{1}, \ldots, \alpha_{K} p_{n}^{K}\right)=\phi\left(\alpha_{1}, \ldots, \alpha_{K}\right) m\left(p_{n}^{1}, \ldots, p_{n}^{K}\right)
$$

for some function $\phi$. Using the properties of $m$, we can deduce that $\phi$ must be continuous, strictly increasing, positive for positive $\alpha_{k}$ with $\phi(1, \ldots, 1)=1$. Equation (A1) is one of Pexider's functional equations and by a result in Eichhorn [1978; 67], there exist positive constants $C, \beta_{1}, \ldots, \beta_{k}$ such that

$m\left(x_{1}, \ldots, x_{K}\right)=C x_{1}^{\beta_{1}} \ldots x_{K}^{\beta_{K}} ; \phi\left(\alpha_{1}, \ldots, \alpha_{K}\right)=\alpha_{1}^{\beta_{1}} \ldots \alpha_{K}^{\beta_{K}}$

Since $m$ is symmetric, all of the $\beta_{k}$ must be equal to a positive constant. Since $m$ is positively linearly homogeneous, each $\beta_{k}$ must equal $1 / K$. Finally, the mean property for $m$ implies $C=1$. Thus

$$
m\left(x_{1}, \ldots, x_{K}\right) \equiv\left[\Pi_{k=1}^{K} x_{k}\right]^{1 / K} .
$$

Hence the symmetric mean multilateral system will satisfy $T 7$ only if $m$ is defined by (A2), which is the geometric average price method defined by (3) and (16).

T9: $\quad \nabla_{y^{k}} S^{k}(P, Y)=[\bar{p} \cdot y]^{-1}\left[1-S^{k}(P, Y)\right] \bar{p}>>0_{N}$

where $y \equiv \Sigma_{k=1}^{K} y^{k}$ and $\bar{p} \equiv\left[m\left(p_{1}^{1}, \ldots, p_{1}^{K}\right), \ldots, m\left(p_{N}^{1}, \ldots, p_{N}^{K}\right)\right]^{T}$.

T10: Part (i) is satisfied but not part (ii).

T11: $\Sigma_{j \in B} S^{j}(P, Y) / \Sigma_{i \in A} S^{i}(P, Y)$ is not independent of the $\alpha_{i}$ and $\gamma_{j}$ and hence is not a function of only $p^{a}, p^{b}, y^{a}$ and $y^{b}$.

Exactness Properties: Assuming (3) and (14), Diewert [1996; 255-256] showed that the only differentiable linearly homogeneous solution to (12) is the $f$ defined by (17) and the only differentiable solution to (13) is the unit cost function $c$ defined by (18).

Proof of Proposition 3: To ensure that the $S^{k}$ defined by (22) are well defined, we assume that all quantity vectors are strictly positive; i.e., we assume $y^{k}>>0_{N}$ for $k=1,2, \ldots, K$. 
T8: Substituting (22) and (23) into the equations defining the test leads to the following system of equations that $m$ must satisfy: for $\lambda_{i}>0, p^{i}>>0_{N}, p^{j}>>0_{N}, y^{k}>>0_{N}$ for $k=1, \ldots, K$ and $1 \leq i \neq j \leq K$ :

$$
\begin{aligned}
\Sigma_{n=1}^{N} p_{n}^{j} m\left(y_{n}^{1}, \ldots, \lambda_{i} y_{n}^{i}, \ldots, y_{n}^{K}\right) / \Sigma_{r=1}^{N} p_{r}^{i} m\left(y_{r}^{1}, \ldots, \lambda_{i} y_{r}^{i}, \ldots, y_{r}^{K}\right) \\
=\sum_{n=1}^{N} p_{n}^{j} m\left(y_{n}^{1}, \ldots, y_{n}^{K}\right) / \Sigma_{r=1}^{N} p_{r}^{i} m\left(y_{r}^{1}, \ldots, y_{r}^{K}\right)
\end{aligned}
$$

Cross multiplying terms in (A3), collecting terms in $p_{n}^{j} p_{r}^{i}$ and choosing a grid of $p^{i}$ and $p^{j}$ vectors implies that equations (A3) will hold only if the following system of equations holds for all $\lambda_{i}>0, i=1, \ldots, K$ and $n, r=1, \ldots, N$ :

$$
m\left(y_{n}^{1}, \ldots, \lambda_{i} y_{n}^{i}, \ldots, y_{n}^{K}\right) / m\left(y_{r}^{1}, \ldots, \lambda_{i} y_{r}^{i}, \ldots, y_{r}^{K}\right)=m\left(y_{n}^{1}, \ldots, y_{n}^{K}\right) / m\left(y_{r}^{1}, \ldots, y_{r}^{K}\right)
$$

Repeated use of (A4) for $i=1, \ldots, K$ implies that the following equation must hold:

$$
\begin{aligned}
m\left(\lambda_{1} y_{n}^{1}, \lambda_{2} y_{n}^{2}, \ldots, \lambda_{K} y_{n}^{K}\right) / m\left(\lambda_{1} y_{r}^{1}, \lambda_{2} y_{r}^{2}, \ldots, \lambda_{K} y_{r}^{K}\right) & \\
= & m\left(y_{n}^{1}, y_{n}^{2}, \ldots, y_{n}^{K}\right) / m\left(y_{r}^{1}, y_{r}^{2}, \ldots, y_{r}^{K}\right)
\end{aligned}
$$

Let $\left(y_{n}^{1}, \ldots, y_{n}^{K}\right) \equiv\left(y_{1}, \ldots, y_{K}\right),\left(y_{r}^{1}, \ldots, y_{r}^{K}\right) \equiv\left(z_{1}, \ldots, z_{K}\right)>>0_{K}$ and $\left(\lambda_{1}, \ldots, \lambda_{K}\right) \equiv$ $\left(z_{1}^{-1}, \ldots, z_{K}^{-1}\right)$. Making these substitutions into (A5) and using $m\left(1_{K}\right)=1$ transforms (A5) into:

$$
m\left(z_{1}^{-1} y_{1}, z_{2}^{-1} y_{2}, \ldots, z_{K}^{-1} y_{K}\right)=m\left(y_{1}, y_{2}, \ldots, y_{K}\right) / m\left(z_{1}, z_{2}, \ldots, z_{K}\right)
$$

Define $g\left(x_{1}, x_{2}, \ldots, x_{K}\right) \equiv 1 / m\left(x_{1}^{-1}, x_{2}^{-1}, \ldots, x_{K}^{-1}\right)$. Letting $x_{k}=z_{k}^{-1}$ for $k=1, \ldots, K$ and using the definition of $g$, (A6) becomes the following functional equation:

$$
m\left(x_{1} y_{1}, x_{2} y_{2}, \ldots, x_{K} y_{K}\right)=m\left(y_{1}, y_{2}, \ldots, y_{K}\right) g\left(x_{1}, x_{2}, \ldots, x_{K}\right)
$$

which must hold for all $x>0_{K}$ and $y>0_{K}$. Now apply a result due to Eichhorn $[1978 ; 67]$ to (A7), use the assumption that $m$ is a homogeneous mean and conclude that $m$ must be defined by (24) in order that (A7) hold. It is straightforward to show that if $m$ is defined by (24), then test $T 8$ holds. Hence a symmetric mean average quantity method will satisfy $T 8$ if and only if the homogeneous symmetric mean $m$ is the geometric mean defined by (24).

T9: Consider first the arithmetic mean case where $S^{k}$ is defined by (22) and (23). A straightforward calculation shows that for $j=2, \ldots, K$ and $z>0_{N}$, we have

$$
S^{1}\left(P, y^{1}+z, y^{2}, \ldots, y^{K}\right) / S^{j}\left(P, y^{1}+z, y^{2}, \ldots, y^{K}\right)>S^{1}(P, Y) / S^{j}(P, Y)
$$


The inequalities (A8) imply that $S^{1}\left(P, y^{1}, y^{2}, \ldots, y^{K}\right)$ is increasing in the components of $y^{1}$. We can similarly show that $S^{k}(P, Y)$ is increasing in the components of $y^{k}$ for any $k$.

Now consider the geometric mean case where $S^{k}$ is defined by (22) and (24). Let $K=2$ and $N=2$ and calculate the derivative of $S^{1}(P, Y) / S^{2}(P, Y)$ with respect to $y_{1}^{1}$. It is possible to find positive vectors $p^{1}, p^{2}, y^{1}, y^{2}$ which make this derivative negative. Hence the geometric weights method fails the monotonicity test $T 9$.

T10: Part (i) holds but part (ii) does not.

T11: $\Sigma_{j \in B} S^{j}(P, Y) / \Sigma_{i \in A} S^{i}(P, Y)$ is not independent of the $\beta_{i}$ and $\delta_{j}$ and hence is not a function of only $p^{a}, p^{b}, y^{a}$ and $y^{b}$.

Exactness Properties: Diewert [1996; 257] showed that for this method, the only differentiable linearly homogeneous solution to (12) is the $f$ defined by (17) and the only differentiable solution to (13) is the unit cost function defined by (18).

\section{Proof of Proposition 4:}

T1: The Perron-Frobenius Theorem implies that the maximal eigenvalue eigenvector of the positive matrix $C$, subject to the normalization (28), is unique and thus the components of $\pi$ will be continuous functions of the elements of $C$ and hence of the elements of $P$ and $Y$.

T3: Let $p>>0_{N}, \alpha_{k}>0$ and $p^{k}=\alpha_{k} p$ for $k=1, \ldots, K$. Define $y \equiv \Sigma_{k=1}^{K} y^{k}$. We need to show that $S^{k}=p \cdot y^{k} / p \cdot y$ for $k=1, \ldots, K$. Hence we need only show that $\pi \equiv p / p \cdot y$ satisfies (27) or equivalently that $C p=p$. We have:

$$
\begin{aligned}
C p & =\hat{y}^{-1} \Sigma_{k=1}^{K} \alpha_{k} \hat{p} y^{k} y^{k T} p / \alpha_{k} p^{T} y^{k} \\
& =\hat{y}^{-1} \hat{p} \Sigma_{k=1}^{K} y^{k} \\
& =\hat{y}^{-1} \hat{y} p \\
& =p .
\end{aligned}
$$

T7: The matrix $C$ defined by (30) remains invariant if $p^{k}$ is replaced by $\alpha_{k} p^{k}$ for $k=1, \ldots, K$.

T8: For this test to pass when $K=2$, we require $P_{G K}$ defined by (32) to be homogeneous of degree 0 in the components of $y^{a}$ which is not true.

T9: When $K=2$, we require that $S^{2} / S^{1}$ be increasing in the components of $y^{2}$. In this case, we obtain an explicit function of $p^{1}, p^{2}, y^{1}, y^{2}$ for $S^{2} / S^{1}$ (see the right hand side of 
(31) with $a=1$ and $b=2$ ) and by differentiating this function with respect to a component of $y^{2}$, we can verify that monotonicity fails.

T10(i): Let $p^{a}>>0_{N}, p^{i}=\alpha_{i} p^{a}, \alpha_{i}>0, y^{a}>0_{N}, y^{i}=\beta_{i} y^{a}, \beta_{i}>0$ for $i \in A$ with $\Sigma_{i \in A} \beta_{i}=1$. For $i \in A$ and $j \in A$, we have

$S^{i}(P, Y) / S^{j}(P, Y)=\pi \cdot y^{i} / \pi \cdot y^{j}=\pi \cdot \beta_{i} y^{a} / \pi \cdot \beta_{j} y^{a}=\beta_{i} / \beta_{j}$.

T10(ii): If we premultiply (27) by the diagonal matrix $\hat{y}$, the resulting system of equations becomes:

$$
\begin{gathered}
{\left[\Sigma_{i \in A} \hat{y}^{i}+\Sigma_{j \in B} \hat{y}^{j}-\Sigma_{i \in A}\left(p^{i} \cdot y^{i}\right)^{-1} \hat{p}^{i} y^{i} y^{i T}-\Sigma_{j \in B}\left(p^{j} \cdot y^{j}\right)^{-1} \hat{p}^{j} y^{j} y^{j T}\right] \pi=0_{N} \text { or }} \\
{\left[\Sigma_{i \in A} \beta_{i} \hat{y}^{a}+\Sigma_{j \in B} \hat{y}^{j}-\Sigma_{i \in A}\left(\alpha_{i} \beta_{i} p^{a} \cdot y^{a}\right)^{-1} \alpha_{i} \hat{p}^{a} \beta_{i} y^{a} \beta_{i} y^{a T}\right.} \\
\left.-\Sigma_{j \in B}\left(p^{j} \cdot y^{j}\right)^{-1} \hat{p}^{j} y^{j} y^{j}\right] \pi=0_{N} \text { or }
\end{gathered}
$$

$$
\left[y^{a}+\Sigma_{j \in B} \hat{y}^{j}-\left(p^{a} \cdot y^{a}\right)^{-1} \hat{p}^{a} y^{a} y^{a T}-\Sigma_{j \in B}\left(p^{j} \cdot y^{j}\right)^{-1} \hat{p}^{j} y^{j} y^{j T}\right] \pi=0_{N} .
$$

If we premultiply (A10) by $\hat{y}^{-1}$, we obtain $\left[I_{N}-C^{*}\right] \pi=0_{N}$ where $C^{*}$ is the matrix that corresponds to the aggregated (over countries in the subbloc $A$ ) model. Hence if $\pi$ satisfies (A9) and (28), it will also satisfy (A10) and (28).

T11: Under the restrictions for this test, the system (27) or equivalently the system (A9) reduces to:

$$
\left[\hat{y}^{a}+\hat{y}^{b}-\left(p^{a} \cdot y^{a}\right)^{-1} \hat{p}^{a} y^{a} y^{a T}-\left(p^{b} \cdot y^{b}\right)^{-1} \hat{p}^{b} y^{b} y^{b T}\right] \pi=0_{N} \quad \text { or }
$$

$$
\left[\hat{y}^{a}+\hat{y}^{b}\right]^{-1}\left[\left(p^{a} \cdot y^{a}\right)^{-1} \hat{p}^{a} y^{a} y^{a T}+\left(p^{b} \cdot y^{b}\right)^{-1} \hat{p}^{b} y^{b} y^{b T}\right] \pi=\pi
$$

Define the vectors of expenditure shares for subblocs $A$ and $B$ by $s^{a} \equiv \hat{p}^{a} y^{a} / p^{a} \cdot y^{a}$ and $s^{b} \equiv \hat{p}^{b} y^{b} / p^{b} \cdot y^{b}$ respectively and the quantity shares of "world" output for the two blocs $A$ and $B$ by $q^{a} \equiv\left[\hat{y}^{a}+\hat{y}^{b}\right]^{-1} y^{a}$ and $q^{b} \equiv\left[\hat{y}^{a}+\hat{y}^{b}\right]^{-1} y^{b}$ respectively. Note that $q^{a}+q^{b}=1_{N}$, a vector of ones. Now premultiply both sides of (A11) by $y^{a T}$. Rearranging terms in the resulting equation, we find that:

$$
\begin{aligned}
& \pi \cdot y^{b} / \pi \cdot y^{a}=\left[1-s^{a} \cdot q^{a}\right] / s^{b} \cdot q^{b} \\
& =\left[s^{a} \cdot 1_{N}-s^{2} \cdot q^{a}\right] / s^{b} \cdot q^{b} \quad \text { since } s^{a} \cdot 1_{N}=1 \\
& =s^{a} \cdot q^{b} / s^{b} \cdot q^{a} \quad \text { since } \quad 1_{N}-q^{a}=q^{b} \\
& =\left\{p^{a T} \hat{y}^{a}\left[\hat{y}^{a}+\hat{y}^{b}\right]^{-1} y^{b} / p^{b T} \hat{y}^{b}\left[\hat{y}^{a}+\hat{y}^{b}\right]^{-1} y^{a}\right\}\left\{p^{b} \cdot y^{b} / p^{a} \cdot y^{a}\right\} \\
& =p^{b} \cdot y^{b} / p^{a} \cdot y^{a} P_{G K}\left(p^{a}, p^{b}, y^{a}, y^{b}\right)
\end{aligned}
$$


where $P_{G K}$ is the bilateral Geary-Khamis price index defined by (32). Since the left hand side of (A12) is $\Sigma_{j \in B} S^{j} / \Sigma_{i \in A} S^{i}$, we see that test $T 11$ fails: we do not obtain the bilateral Fisher ideal quantity index on the right hand side of (A12).

Exactness Properties: We first consider the two country case, $K=2$. In this case when we have differentiable demand functions, using (A12), equations (13) reduce to the following single equation:

$$
\begin{aligned}
p^{2} \cdot y^{2} / p^{1} \cdot y^{1} P_{G K}\left(p^{1}, p^{2}, y^{1}, y^{2}\right) & =u_{2} / u_{1} \quad \text { or } \\
c\left(p^{2}\right) u_{2} / c\left(p^{1}\right) u_{1} P_{G K}\left(p^{1}, p^{2}, \nabla c\left(p^{1}\right) u_{1}, \nabla c\left(p^{2}\right) u_{2}\right) & =u_{2} / u_{1} \quad \text { or } \\
P_{G K}\left(p^{1}, p^{2}, \nabla c\left(p^{1}\right) u_{1}, \nabla c\left(p^{2}\right) u_{2}\right) & =c\left(p^{2}\right) / c\left(p^{1}\right) \quad \text { or } \\
\frac{\left.\sum_{n=1}^{N} p_{n}^{2} c_{n}\left(p^{1}\right) c_{n}\left(p^{2}\right) u_{1} u_{2} / \mid c_{n}\left(p^{1}\right) u_{1}+c_{n}\left(p^{2}\right) u_{2}\right]}{\sum_{m=1}^{N} p_{m}^{1} c_{m}\left(p^{1}\right) c_{m}\left(p^{2}\right) u_{1} u_{2} /\left|c_{m}\left(p^{1}\right) u_{1}+c_{m}\left(p^{2}\right) u_{2}\right|} & =\frac{c\left(p^{2}\right)}{c\left(p^{1}\right)}
\end{aligned}
$$

The left hand side of (A13) is independent of $u_{1}$ and $u_{2}$ only if $c_{n}\left(p^{1}\right)=c_{n}\left(p^{2}\right)$ for $n=1,2, \ldots, N$ for all price vectors $p^{1}$ and $p^{2}$; i.e., the first order partial derivatives of the unit cost function $c(p)$ must be constant in order for (13) to hold. Hence in the case of differentiable demand functions and only 2 countries, the unit cost function must be linear.

Now consider the two country case with differentiable inverse demand functions. In this case, equations (12) reduce to the following single equation:

$$
\begin{aligned}
f\left(y^{2}\right) e_{2} / f\left(y^{1}\right) e_{1} P_{G K}\left(\nabla f\left(y^{1}\right) e_{1}, \nabla f\left(y^{2}\right) e_{2}, y^{1}, y^{2}\right) & =f\left(y^{2}\right) / f\left(y^{1}\right) \text { or } \\
y^{2 T}\left[\hat{y}^{1}+\hat{y}^{2}\right]^{-1} \hat{y}^{1} \nabla f\left(y^{2}\right) e_{2} / y^{2 T}\left[\hat{y}^{1}+\hat{y}^{2}\right]^{-1} \hat{y}^{1} \nabla f\left(y^{1}\right) e_{1} & =e_{2} / e_{1} \quad \text { or } \\
y^{2 T}\left[\hat{y}^{1}+\hat{y}^{2}\right]^{-1} \hat{y}^{1}\left[\nabla f\left(y^{2}\right)-\nabla f\left(y^{1}\right)\right] & =0 .
\end{aligned}
$$

For $n=1,2, \ldots, N$, set $y^{1}=i_{n}$, the $n$th unit vector, and substitute into (A14). We find that we must have $f_{n}\left(y^{2}\right)=f_{n}\left(i_{n}\right)$ for all $y^{2}$ for $n=1, \ldots, N$. Hence the first order partial derivatives of $f$ are constant and the only solution to (12) is the linear $f$ defined by (17) in the two country case.

Now consider the case where $K \geq 3$. In the case of differentiable demand functions, using (8) and (29), equations (13) reduce to:

$$
\begin{aligned}
\pi \cdot \nabla c\left(p^{i}\right) u_{i} / \pi \cdot \nabla c\left(p^{j}\right) u_{j} & =u_{i} / u_{j} & & \text { or } \\
\pi \cdot\left[\nabla c\left(p^{i}\right)-\nabla c\left(p^{j}\right)\right] & =0 & & \text { for } 1 \leq i, j \leq K .
\end{aligned}
$$

Recall that the equations which define $\pi$ are (27) and (28). Using (8) and letting ` denote the operation of diagonalizing a vector into a matrix, (27) is equivalent to: 


$$
\left[\Sigma_{m=1}^{K} \nabla \hat{c}\left(p^{m}\right) u_{m}-\Sigma_{m=1}^{K}\left[c\left(p^{m}\right)\right]^{-1} \hat{p}^{m} \nabla c\left(p^{m}\right) \nabla^{T} c\left(p^{m}\right) u_{m}\right] \pi=0_{N}
$$

To show that (A16) implies that $\nabla c\left(p^{i}\right)=\nabla c\left(p^{j}\right)$ for all $p^{i}$ and $p^{j}$ (and hence that $c$ must be defined by (18)), we need only show that by varying $p^{k}$ (where $k$ is not equal to $i$ or $j$ ), we can find $N$ linearly independent $\pi^{k}$ which satisfy (A15). By examining (A16), we see that this can be done. If we let all of the $u_{m}$ in (A16) be close to 0 except for $u_{k}$, then (A16) is approximately equivalent to the following system of equations:

$$
\begin{aligned}
& \pi_{n} c_{n}\left(p^{k}\right) u_{k}-p_{n}^{k} c_{n}\left(p^{k}\right) \nabla c\left(p^{k}\right) \cdot \pi / c(p) u_{k}=0 ; \quad n=1, \ldots, N, \text { or } \\
& \pi_{n}=p_{n}^{k} \pi \cdot \nabla c\left(p^{k}\right) / c\left(p^{k}\right) ; \quad n=1, \ldots, N .
\end{aligned}
$$

Hence $\pi$ is proportional to $p^{k}$ and by choosing $N$ linearly independent $p^{k}$ vectors, we can obtain $N$ linearly independent $\pi^{k}$ vectors.

For the case of differentiable inverse demand functions when $K \geq 3$, a proof of exactness in Diewert $[1996 ; 257]$ can be adapted to the present situation to show that the differentiable linearly homogeneous aggregator function $f$ must be the linear one defined by (17).

\section{Proof of Proposition 5:}

T1: By the Perron-Frobenius Theorem, the maximum eigenvalue right eigenvector $s$ of the positive matrix $D$, subject to the normalization (36), is strictly positive and unique. Thus $s$ will be a continuous function of the elements of $D$ and hence of the components of $P$ and $Y$.

T2: Under the assumptions for this test, equations (39) become

$$
S^{i}=\alpha \Sigma_{j=1}^{J}\left(\beta_{i} / \beta_{j}\right) S^{j} \quad \text { for } i=1, \ldots, K .
$$

Thus $\alpha=1 / K, S^{k}=\beta_{k}$ for $k=1, \ldots, K$ satisfy (36) and (39).

T3: Let $p>>0_{N}, \alpha_{k}>0, p^{k}=\alpha_{k} p$ for $k=1, \ldots, K$. Equations (39) become

$$
\begin{array}{rlrl}
S^{i} & =\alpha \Sigma_{j=1}^{K}\left(\alpha_{j} p \cdot y^{i} / \alpha_{j} p \cdot y^{j}\right) S^{j} & & \text { for } i=1, \ldots, K \\
S^{i} / p \cdot y^{i} & =\alpha \Sigma_{j=1}^{k} S^{j} / p \cdot y^{j} & & \text { or } \\
& \text { for } i=1, \ldots, K .
\end{array}
$$

Hence $\alpha \equiv 1 / K$ and $S^{k} \equiv p \cdot y^{k} / p \cdot y$ will satisfy (36) and (39).

T4: From equations (36) and (39), $s$ and $\alpha$ are determined by the elements of the matrix $D$. Since these elements are invariant to changes in the units of measurement, so are the elements of $s$. 
T5: Since the elements of $D$ remain unchanged if we change the ordering of the commodities, the elements of $s$ will also remain unchanged.

T6: By examining equations (39), we see that changing the ordering of the countries simply changes the ordering of the elements of $s$ and the maximum positive eigenvalue of $D$ remains unchanged by a simultaneous permutation of its rows and columns.

T9: For $K=2$, equations (39) can be rewritten as follows:

$$
\begin{aligned}
1 & =\alpha\left[1+\left(p^{2} \cdot y^{1} / p^{2} \cdot y^{2}\right)\left(S^{2} / S^{1}\right)\right] \\
S^{2} / S^{1} & =\alpha\left[\left(p^{1} \cdot p^{2} / p^{1} \cdot y^{1}\right)+\left(S^{2} / S^{1}\right)\right] .
\end{aligned}
$$

Eliminating $\alpha$ from the above two equations leads to the following single equation:

$$
S^{2} / S^{1}=\left[p^{1} \cdot y^{2} p^{2} \cdot y^{2} / p^{1} \cdot y^{1} p^{2} \cdot y^{1}\right]^{\frac{1}{2}} \equiv Q_{F}\left(p^{1}, p^{2}, y^{1}, y^{2}\right) .
$$

Note that $S^{2} / S^{1}=Q_{F}\left(p^{1}, p^{2}, y^{1}, y^{2}\right)$ is increasing in the components of $y^{2}$ and decreasing in the components of $y^{1}$. Thus for $K=2$, monotonicity is satisfied.

However, for $K \geq 3$, monotonicity is not satisfied in general. The $K+1$ equations which define the $K$ dimensional vector of shares $s$ and the maximum positive eigenvalue $\lambda$ of $D$ are (recall (36) and (40) with $\lambda \equiv 1 / \alpha$ ):

$$
\left[D-\lambda I_{K}\right] s=0_{K} ; \quad 1_{K} \cdot s=1
$$

where $d_{i j} \equiv p^{j} \cdot y^{i} / p^{j} \cdot y^{j}$ for $i, j=1, \ldots, K$. Note that $d_{i i}=1$ for all $i$. When $K=3, \lambda$ is the maximal root of the determinantal equation $\left|D-\lambda I_{3}\right|=0$. Define $x \equiv 1-\lambda$ and this determinantal equation becomes

$$
x^{3}-\left[d_{12} d_{21}+d_{31} d_{13}+d_{32} d_{23}\right] x+\left[d_{12} d_{23} d_{31}+d_{13} d_{21} d_{32}\right]=0 .
$$

We need to find the smallest real root of the above equation. In order to find an explicit solution, consider the case where $d_{13}=d_{23}=0$. In this case, we find that $\lambda=1+\left[d_{12} d_{21}\right]^{\frac{1}{2}}$. Substitute this value for $\lambda$ into (A17) when $K=3$ to determine the components of $s \equiv\left[S^{1}, S^{2}, S^{3}\right]^{T}$ :

$$
S^{1}=\left(d_{21}\right)^{\frac{1}{2}} d_{12} / D ; S^{2} \equiv\left(d_{12}\right)^{\frac{1}{2}} d_{21} / D ; S^{3} \equiv\left[\left(d_{12}\right)^{\frac{1}{2}} d_{31}+\left(d_{21}\right)^{\frac{1}{2}} d_{32}\right] / D
$$

with $D \equiv\left(d_{21}\right)^{\frac{1}{2}} d_{12}+\left(d_{12}\right)^{\frac{1}{2}} d_{21}+\left(d_{12}\right)^{\frac{1}{2}} d_{31}+\left(d_{21}\right)^{\frac{1}{2}} d_{32}>0$. By substituting $d_{i j} \equiv$ $p^{j} \cdot y^{i} / p^{j} \cdot y^{j}$ into (A18) and differentiating $S^{1}$ with respect to the components of $y^{1}$, it can be verified that $S^{1}$ is not always increasing in the components of $y^{1}$.

T10: Part (i) is satisfied but part (ii) is not. 
T11: Substitute the assumptions of the test into equations (39). Then for $i \in A$, (39) reduces to (A19) below and for $j \in B$, (39) reduces to (A20):

$$
\begin{aligned}
& S^{i}=\alpha \Sigma_{k \in A}\left(\alpha_{k} p^{a} \cdot \beta_{k} y^{a}\right)^{-1} \alpha_{k} p^{a} \cdot \beta_{i} y^{a} S^{k}+\alpha \Sigma_{j \in B}\left(\gamma_{j} p^{b} \cdot \delta_{j} y^{b}\right)^{-1} \gamma_{j} p^{b} \cdot \beta_{i} y^{a} S^{j} \\
& S^{j}=\alpha \Sigma_{i \in A}\left(\alpha_{i} p^{a} \cdot \beta_{i} y^{a}\right)^{-1} \alpha_{i} p^{a} \cdot \gamma_{j} y^{b} S^{i}+\alpha \Sigma_{k \in B}\left(\gamma_{k} p^{b} \cdot \delta_{k} y^{b}\right)^{-1} \gamma_{k} p^{b} \cdot \delta_{j} y^{b} S^{k}
\end{aligned}
$$

Now let $S^{i}=\beta_{i} S^{a}$ for $i \in A$ and $S^{j}=\gamma_{j} S^{b}$ for $j \in B$. Substituting these equations into (A19) and (A20), we find that each equation in (A19) reduces to (A21) and each equation in (A20) reduces to the single equation (A22):

$$
\begin{aligned}
& S^{a}=\alpha(\# A)\left(p^{a} \cdot y^{a} / p^{a} \cdot y^{a}\right) S^{a}+\alpha(\# B)\left(p^{b} \cdot y^{a} / p^{b} \cdot y^{b}\right) S^{b} \\
& S^{b}=\alpha(\# A)\left(p^{a} \cdot y^{b} / p^{a} \cdot y^{a}\right) S^{a}+\alpha(\# B)\left(p^{b} \cdot y^{b} / p^{b} \cdot y^{b}\right) S^{b}
\end{aligned}
$$

where $\# A$ is the number of countries in $A$ and $\# B$ is the number of countries in $B$. Eliminating $\alpha$ from (A21) and (A22), we obtain the following single equation in $S^{b} / S^{a}$ :

$$
\beta\left(p^{b} \cdot y^{a} / p^{b} \cdot y^{b}\right) Q^{2}+(1-\beta) Q-\left(p^{a} \cdot y^{b} / p^{a} \cdot y^{a}\right)=0
$$

where $\beta \equiv \# B / \# A$ and $Q \equiv S^{b} / S^{a}$. If $\# A=\# B$ and hence $\beta=1$, the $Q$ solution to (A23) reduces to $Q=Q_{F}\left(p^{a}, p^{b}, y^{a}, y^{b}\right)=S^{b} / S^{a}$. However, in general the number of countries in each subbloc $A$ and $B$ is not restricted to be the same and so in general test T11 fails.

Exactness Properties: When $K=2$, in the proof of $T 9$, we established a result due to Van Yzeren [1957; 15]; namely $S^{2} / S^{1}=Q_{F}\left(p^{1}, p^{2}, y^{1}, y^{2}\right)$, the Fisher quantity index. Thus (41) and (42) are exact for this method when $K=2$.

For $K \geq 3$, replace $S^{j} / S^{i}$ by $f\left(y^{j}\right) / f\left(y^{i}\right)$ and $p^{j}$ by $\nabla f\left(y^{j}\right) e_{j}$ in equations (39). Letting $\lambda=1 / \alpha$, the transformed equations (39) become, using $y^{k} \cdot \nabla f\left(y^{k}\right)=f\left(y^{k}\right)$ :

$$
\begin{aligned}
\Sigma_{k=1}^{K}\left[y^{i} \cdot \nabla f\left(y^{k}\right) e_{k} / f\left(y^{k}\right) e_{k}\right]\left[f\left(y^{k}\right) / f\left(y^{i}\right)\right] & =\lambda ; & i=1, \ldots, K \quad \text { or } \\
\Sigma_{K=1}^{k} y^{i} \cdot \nabla f\left(y^{k}\right) / f\left(y^{i}\right) & =\lambda ; & i=1, \ldots, K
\end{aligned}
$$

Let $j \neq i$ and subtract equation $j$ in (A24) from equation $i$. We obtain the following system of equations for $i \neq j$ :

$$
\Sigma_{k=1}^{K} \nabla f\left(y^{k}\right) \cdot\left\{\left[y^{i} / f\left(y^{i}\right)\right]-\left[y^{j} / f\left(y^{j}\right)\right]\right\}=0 .
$$

If $f$ is the linear aggregator function defined by (17), it is easy to verify that this $f$ satisfies (A25) (and (A24) with $\lambda=K$ ). For $K \geq 3$, we now show that this is the only solution to (A25). 
Let $f$ be linearly homogeneous, increasing and once continuously differentiable and let $f$ satisfy (A25). Suppose that the first order partial derivatives of $f$ are not all constant. Then we can find two strictly positive vectors $y^{(1)}$ and $y^{(2)}$ such that $\nabla f_{r}\left(y^{(1)}\right)$ and $\nabla f\left(y^{(2)}\right)$ are linearly independent, nonnegative and nonzero vectors. Pick commodities $r$ and $s$ such that the vectors $\left[f_{r}\left(y^{(1)}\right), f_{s}\left(y^{(1)}\right)\right]$ and $\left[f_{r}\left(y^{(2)}\right), f_{s}\left(y^{(2)}\right)\right]$ are linearly independent. Fix $i$ and $j$ with $i \neq j$ and choose $y_{n}^{i}=y_{n}^{j}$ for all $n$ except when $n=r$ or $n=s$. For the $r$ and $s$ components of $y^{i}$ and $y^{j}$, choose $y_{r}^{i}, y_{s}^{i}, y_{r}^{j}, y_{s}^{j}$ such that $f\left(y^{i}\right)=f\left(y^{j}\right)$ and

$$
\begin{aligned}
& \left\{y_{r}^{j} / f\left(y^{j}\right)\right\}-\left\{y_{r}^{i} / f\left(y_{i}\right)\right\} \equiv z_{r}>0 \\
& \left\{y_{s}^{j} / f\left(y^{j}\right)\right\}-\left\{y_{s}^{i} / f\left(y^{i}\right)\right\} \equiv z_{s} \leq 0
\end{aligned}
$$

Substitute these choices for $y^{i}$ and $y^{j}$ into (A25) to obtain:

$$
\left[\Sigma_{k=1}^{K} f_{\mathrm{r}}\left(y^{k}\right)\right] z_{\mathrm{r}}+\left[\Sigma_{k=1}^{K} f_{s}\left(y^{k}\right)\right] z_{s}=0 .
$$

Since $K \geq 3$, there exists a country $k$ not equal to $i$ or $j$. For such a $k$, replace $y^{k}$ in (A27) by $y^{(1)}$ and then $y^{(2)}$. Rewrite the resulting two equations as

$$
x_{11} z_{\tau}+x_{12} z_{s}=0 ; x_{21} z_{r}+x_{22} z_{s}=0 \text {. }
$$

Note that the vectors $\left[x_{11}, x_{12}\right]$ and $\left[x_{21}, x_{22}\right]$ are equal to the linearly independent vectors $\left[f_{r}\left(y^{(1)}, f_{s}\left(y^{(1)}\right]\right.\right.$ and $\left[f_{r}\left(y^{(2)}, f_{s}\left(y^{(2)}\right]\right.\right.$ plus a common vector. Since the first order partial derivatives of are continuous, we can perturb $y^{(1)}$ and $y^{(2)}$ slightly if necessary to ensure the linear independence of $\left[x_{11}, x_{12}\right]$ and $\left[x_{21}, x_{22}\right]$. The linear independence of these two vectors and (A28) implies that $z_{r}=0$ and $z_{s}=0$ which contradicts (A26). Thus our supposition that the first order partial derivatives of $f$ are not all constant leads to a contradiction.

We now determine what unit cost functions $c$ are consistent with equations (39). Substituting (9) and (13) into (39) and letting $\lambda=1 / \alpha$ leads to the following system of functional equations:

$$
\begin{aligned}
\Sigma_{k=1}^{K}\left[p^{k} \cdot \nabla c\left(p^{i}\right) u_{i} / c\left(p^{k}\right) u_{k}\right]\left[u_{k} / u_{i}\right]=\lambda & \text { for } \quad i=1, \ldots, K \text { or } \\
\Sigma_{k=1}^{K} p^{k} \cdot \nabla c\left(p^{i}\right) / c\left(p^{k}\right)=\lambda & \text { for } \quad i=1, \ldots, K .
\end{aligned}
$$

Let $j \neq i$ and subtract equation $j$ in (A29) from equation $i$. We obtain the following system of equations for $1 \leq i \neq j \leq K$ :

$$
\Sigma_{k=1}^{K}\left[p^{k T} / c\left(p^{k}\right)\right]\left[\nabla c\left(p^{i}\right)-\nabla c\left(p^{j}\right)\right]=0 .
$$


Since $K \geq 3$, there exists an $m$ not equal to $i$ or $j$. Choose $N p^{m}$ vectors, say $p^{m n}, n=$ $1, \ldots, N$, such that the vectors $p^{m n} / c\left(p^{m n}\right)+\Sigma_{k=1, k \neq m}^{K}\left[p^{k} / c\left(p^{k}\right)\right], n=1, \ldots, N$, are linearly independent. Substitute these $p^{m n}$ into (A30) and we deduce that $\nabla c\left(p^{i}\right)=\nabla c\left(p^{j}\right)$ for all $p^{i}$ and $p^{j}$. Hence the first order partial derivatives of $c$ must be constants. Using the fact that $c$ must be linearly homogeneous, we further deduce that $c$ must be the linear unit cost function defined by (18).

\section{Proof of Proposition 6:}

T1: By the Perron-Frobenius Theorem, the maximum eigenvalue left eigenvector, $\left[\left(S^{1}\right)^{-1}\right.$, $\left.\ldots,\left(S^{K}\right)^{-1}\right]^{T}$, of the positive matrix $D$, subject to the normalization (46), is strictly positive and unique. Thus $\left[\left(S^{1}\right)^{-1}, \ldots,\left(S^{K}\right)^{-1}\right]$ and hence $\left[S^{1}, \ldots, S^{K}\right]$ will be continuous functions of the elements of $D$ and hence of the components of $P$ and $Y$.

T2: Let $y^{k}=\beta_{k} y, y>>0_{N}, \beta_{k}>0$ for $k=1, \ldots, K$ with $\Sigma_{k=1}^{K} \beta_{k}=1$. Equations (49) become

$$
\left(S^{i}\right)^{-1}=\alpha \Sigma_{k=1}^{K}\left[\beta_{k} / \beta_{i}\right]\left(S^{k}\right)^{-1} ; \quad i=1, \ldots, K
$$

Thus $\alpha=1 / K, S^{k}=\beta_{k}$ satisfy (46) and (49).

T3: Let $p>>0_{n}, \alpha_{k}>0, p^{k}=\alpha_{k} p$ for $k=1, \ldots, K$. Equations (49) become

$$
\begin{aligned}
\left(S^{i}\right)^{-1} & =\alpha \Sigma_{k=1}^{K}\left[\alpha_{i} p \cdot y^{k} / \alpha_{i} p \cdot y^{i}\right]\left(S^{k}\right)^{-1} ; \quad i=1, \ldots, K \text { or } \\
p \cdot y^{i} / S^{i} & =\alpha \Sigma_{k=1}^{K} p \cdot y^{k} / S^{k} ; \quad i=1, \ldots, K .
\end{aligned}
$$

Hence $\alpha=1 / K$ and $S^{k}=p \cdot y^{k} / p \cdot \Sigma_{j=1}^{K} y^{j}$ will satisfy (46) and (49).

T4 - T6: Similar to the proofs of T4 - T6 in Proposition 5.

T9: For $K=2$, equations (49) can be written as follows:

$$
\begin{aligned}
1 & =\alpha\left[1+\left(p^{1} \cdot y^{2} / p^{1} \cdot y^{1}\right)\left(S^{1} / S^{2}\right)\right] ; \\
S^{1} / S^{2} & =\alpha\left[\left(p^{2} \cdot y^{1} / p^{2} \cdot y^{2}\right)+\left(S^{1} / S^{2}\right)\right] .
\end{aligned}
$$

Eliminating $\alpha$ from the above two equations leads to $S^{2} / S^{1}=Q_{F}\left(p^{1}, p^{2}, y^{1}, y^{2}\right)$. Thus in the two country case, Van Yzeren's unweighted average basket method leads to the Fisher ideal quantity index which satisfies monotonicity in quantities. However, for $K \geq 3$, we can proceed as in the proof of $T 9$ for Proposition 5 and demonstrate that monotonicity does not always hold.

T10: Part (i) is satisfied but part (ii) is not. 
T11: The proof is analogous to the proof of $T 11$ in Proposition 5. If the number of countries in the subbloc $A$ is equal to the number of countries in the subbloc $B$, then $\Sigma_{j \in B} S^{j} / \Sigma_{i \in A} S^{i}=Q_{F}\left(p^{a}, p^{b}, y^{a}, y^{b}\right)$, but in general, this equality does not hold.

Exactness Properties: For $K=2$, the exactness properties are the usual ones for the Fisher ideal quantity index (see the proof of Proposition 5 above).

For $K \geq 3$, replace $S^{i} / S^{j}$ by $f\left(y^{i}\right) / f\left(y^{j}\right)$ and $p^{j}$ by $\nabla f\left(y^{j}\right) e_{j}$. Letting $\lambda=1 / \alpha$, the transformed equations (49) become:

$$
\begin{aligned}
\Sigma_{k=1}^{K}\left[y^{k} \cdot \nabla f\left(y^{i}\right) e_{i} / f\left(y^{i}\right) e_{i}\right]\left[f\left(y^{i}\right) / f\left(y^{k}\right)\right] & =\lambda ; & & i=1, \ldots, K \quad \text { or } \\
\Sigma_{k=1}^{K} y^{k} \cdot \nabla f\left(y^{i}\right) / f\left(y^{k}\right) & =\lambda ; & & i=1, \ldots, K .
\end{aligned}
$$

Let $j \neq i$ and subtract equation $j$ in (A31) from equation $i$. We obtain the following system of functional equations for $i \neq j$ :

$$
\Sigma_{k=1}^{K}\left[y^{k T} / f\left(y^{k}\right)\right]\left[\nabla f\left(y^{i}\right)-\nabla f\left(y^{j}\right)\right]=0 ; \quad 1 \leq i \neq j \leq K .
$$

This is the same system of functional equations as (A30) except that $f$ replaces $c$ and $y^{k}$ replaces $p^{k}$. Thus the only differentiable linearly homogeneous solution to (A31) is the linear aggregator function defined by (17).

Similarly, substituting (9) and (13) into (49) and letting $\lambda=1 / \alpha$ leads to the following system of functions equations:

$$
\begin{aligned}
& \Sigma_{k=1}^{K}\left[p^{i} \cdot \nabla c\left(p^{k}\right) u_{k} / c\left(p^{i}\right) u_{i}\right]\left[u_{i} / u_{k}\right]=\lambda ; \quad i=1, \ldots, K \text { or } \\
& \Sigma_{k=1}^{K} p^{i} \cdot \nabla c\left(p^{k}\right) / c\left(p^{i}\right)=\lambda ; \quad i=1, \ldots, K .
\end{aligned}
$$

Let $j \neq i$ and subtract equation $j$ in (A33) from equation $i$. We obtain:

$$
\Sigma_{k=1}^{K} \nabla c\left(p^{k}\right) \cdot\left\{\left[p^{i} / c\left(p^{i}\right)\right]-\left[p^{j} / c\left(p^{j}\right)\right]\right\}=0 ; \quad i \leq i \neq j \leq K .
$$

The system (A34) is identical to (A25) except that $c$ replaces $f$ and $p^{k}$ replaces $y^{k}$. Thus as usual, we deduce that the only linearly homogeneous solution to (A34) is the Leontief unit cost function defined by (18).

Proof of Proposition 7: We restrict the domain of definition to strictly positive quantity vectors. Using the positivity test BT1 and the circularity test (52), we have, following Eichhorn [1978; 67]:

$$
Q\left(p^{1}, p^{2}, y^{1}, y^{2}\right)=Q\left(p^{0}, p^{2}, y^{0}, y^{2}\right) / Q\left(p^{0}, p^{1}, y^{0}, y^{1}\right)=h\left(p^{2}, y^{2}\right) / h\left(p^{1}, y^{1}\right)
$$

where we fixed $p^{0}$ and $y^{0}$ and defined $h(p, y) \equiv Q\left(p^{0}, p, y^{0}, y\right)$. Now let $y^{1}=y^{2}=y$ in (A35) and applying the identity test BT3, we find that $h\left(p^{1}, y\right)=h\left(p^{2}, y\right)$ for all $p^{1}>>0$ 
and $p^{2}>>0$, which means $h(p, y)$ is independent of $p$. Defining $m(y) \equiv h\left(1_{N}, y\right),($ A35) becomes

$$
Q\left(p^{1}, p^{2}, y^{1}, y^{2}\right)=m\left(y^{2}\right) / m\left(y^{1}\right) .
$$

Now apply commensurability BT10 to (A36) with $\delta_{n} \equiv y_{n}^{1}$ for $n=1, \ldots, N$. We obtain

$$
m\left(y^{2}\right) / m\left(y^{1}\right)=m\left[\left(y_{1}^{1}\right)^{-1} y_{1}^{2}, \ldots,\left(y_{N}^{1}\right)^{-1} y_{N}^{2}\right] / m\left(1_{N}\right) .
$$

Define $g\left(x_{1}, \ldots, x_{N}\right) \equiv m\left(1_{N}\right) / m\left(x_{1}^{-1}, \ldots, x_{N}^{-1}\right)$ and (A37) becomes the functional equation (A7), with $N$ replacing $K$. Note that the monotonicity in quantities test BT12 implies that $m$ and $g$ are strictly increasing functions. Hence we may apply Eichhorn's $[1978 ; 66-68]$ Theorem (noting that $g\left(1_{N}\right)=1$ ) and conclude that

$$
m\left(y_{1}, \ldots, y_{N}\right)=\beta y_{1}^{\alpha_{1}} \ldots y_{N}^{\alpha_{N}}
$$

Setting $y^{1}=y^{2}=1_{N}$ in (A37) and using BT3 implies that $\left[m\left(1_{N}\right)\right]^{2}=1$. Using BT1, $m\left(1_{N}\right)=1$ and hence the $\beta$ in (A38) must equal 1. The monotonicity test BT12 implies that each $\alpha_{n}$ is positive and the linear homogeneity test BT5 implies that the $\alpha_{n}$ sum to 1.

\section{Proof of Proposition 8:}

T1: Using (58), BT1 and BT2, it is evident that $T 1$ is satisfied.

T2: Let $y>>0_{N}, \beta_{k}>0, y^{k}=\beta_{k} y$ for $k=1, \ldots, K$ with $\Sigma_{k=1}^{K} \beta_{k}=1$. Then

$$
\begin{aligned}
Q\left(p^{i}, p^{k}, y^{i}, y^{k}\right) & =Q\left(p^{i}, p^{k}, \beta_{i} y, \beta_{k} y\right) & & \\
& =\left(\beta_{k} / \beta_{i}\right) Q\left(p^{i}, p^{k}, y, y\right) & & \text { using BT5 and BT6 } \\
& =\beta_{k} / \beta_{i} & & \text { using BT3. }
\end{aligned}
$$

Substituting the above into (58), we obtain $S_{k}^{G}=\beta_{k} \alpha /\left[\beta_{1} \ldots \beta_{K}\right]^{1 / K}$ for $k=1, \ldots, K$. Thus $\alpha \equiv\left[\beta_{1} \ldots \beta_{K}\right]^{1 / K}$ and $S_{k}^{G}=\beta_{k}$ as required.

T3: Let $p>>0_{N}, \alpha_{k}>0, p^{k}=\alpha_{k} p$ for $k=1, \ldots, K$. Then

$$
\begin{array}{rlrl}
Q\left(p^{i}, p^{k}, y^{i}, y^{k}\right) & =Q\left(\alpha_{i} p, \alpha_{k} p, y^{i}, y^{k}\right) & \\
& =Q\left(p, p, y^{i}, y^{k}\right) & & \text { using BT7 and BT8 } \\
& =p \cdot y^{k} / p \cdot y^{i} & & \text { using BT4. }
\end{array}
$$

Substituting the above into (58), we obtain 


$$
\begin{aligned}
S_{k}^{G} & =\alpha\left[\Pi_{i=1}^{K} p \cdot y_{k} / p \cdot y^{i}\right]^{1 / K} \\
& =\alpha p \cdot y^{k} /\left[p \cdot y^{1} \ldots p \cdot y^{K}\right]^{1 / K} \quad \text { for } k=1, \ldots, K
\end{aligned}
$$

Thus $S_{k}^{G}$ is proportional to $p \cdot y^{k}$ and $T 3$ is satisfied.

T4: This test follows using (58) and BT10.

T5: This test follows using (58) and BT9.

T6: This test follows from the symmetric nature of (58).

T7: Let $\alpha_{k}>0$ for $k=1, \ldots, K$. Consider equations (58) when $p^{k}$ is replaced by $\alpha_{k} p^{k}$ for $k=1, \ldots, K$ :

$$
\begin{aligned}
S_{k}^{G}\left(\alpha_{1} p^{1}, \ldots, \alpha_{K} p^{K}, Y\right) & =\alpha\left[\Pi_{i=1}^{K} Q\left(\alpha_{i} p^{i}, \alpha_{k} p^{k}, y^{i}, y^{k}\right)\right]^{1 / K} \\
& =\alpha\left[\Pi_{i=1}^{K} Q\left(p^{i}, p^{k}, y^{i}, y^{k}\right)\right]^{1 / K} \quad \text { using BT7 and BT8 } \\
& =S_{k}^{G}\left(p^{1}, \ldots, p^{K}, Y\right) .
\end{aligned}
$$

T8: Let $\lambda>0$ and use (58) to obtain a formula for the following share ratio:

$$
\begin{aligned}
S_{1}^{G}\left(P, \lambda y^{1}, y^{2}, \ldots, y^{K}\right) / S_{2}^{G}\left(P, \lambda y^{1}, y^{2}, \ldots, y^{K}\right) \\
=\frac{\left[Q\left(p^{1}, p^{1}, \lambda y^{1}, \lambda y^{1}\right) \Pi_{i=2}^{K} Q\left(p^{i}, p^{1}, y^{i}, \lambda y^{1}\right)\right]^{1 / K}}{\left[Q\left(p^{1}, p^{2}, \lambda y^{1}, y^{2}\right) \Pi_{j=2}^{K} Q\left(p^{j}, p^{2}, y^{j}, y^{2}\right)\right]^{1 / K}} \\
=\left[\lambda^{K-1} \Pi_{i=1}^{K} Q\left(p^{i}, p^{1}, y^{i}, y^{1}\right) / \lambda^{-1} \Pi_{j=1}^{K} Q\left(p^{j}, p^{2}, y^{j}, y^{2}\right)\right]^{1 / K} \\
\quad \text { using BT5 and BT6 } \\
=\lambda S_{1}^{G}\left(P, y^{1}, y^{2}, \ldots, y^{K}\right) / S_{2}^{G}\left(P, y^{1}, y^{2}, \ldots, y^{K}\right) .
\end{aligned}
$$

The proof for the other share ratios follows in an analogous manner.

T9: Using (58), BT3, BT12 and BT13, we see that if any component of $y^{k}$ increases, then $S_{k}^{G} / \alpha$ increases and the other $S_{j}^{G} / \alpha$ decrease. Hence, using (54), $S_{k}^{G}$ will increase as any component of $y^{k}$ increases.

T10(i): Under the hypotheses of the test, for $k \in A$, we have, using (58):

$$
\begin{array}{rlr}
S_{k}^{G} & =\alpha\left[\Pi_{i=1}^{K} Q\left(p^{i}, p^{k}, y^{i}, y^{k}\right)\right]^{1 / K} & \\
& =\alpha\left[\Pi_{i \in A} Q\left(\alpha_{i} p^{a}, \alpha_{k} p^{a}, \beta_{i} y^{a}, \beta_{k} y^{a}\right) \Pi_{j \in B} Q\left(p^{j}, \alpha_{k} p^{a}, y^{j}, \beta_{k} y^{a}\right)\right]^{1 / K} \\
& =\alpha\left[\Pi_{i \in A}\left(\beta_{k} / \beta_{i}\right) Q\left(p^{a}, p^{a}, y^{a}, y^{a}\right) \Pi_{j \in B} Q\left(p^{j}, p^{a}, y^{j}, y^{a}\right)\right]^{1 / K} & \text { using BT5- } \\
& =\beta_{k} \alpha\left[\Pi_{j \in B} Q\left(p^{j}, p^{a}, y^{j}, y^{a}\right) / \Pi_{i \in A} \beta_{i}\right]^{1 / K} & \text { using BT3. }
\end{array}
$$


Therefore, for $i \in A, j \in A$, we have $S_{i}^{G} / S_{j}^{G}=\beta_{i} / \beta_{j}$. Hence part (i) of $T 10$ passes. However, part (ii) fails.

T11: Under the conditions of the test, for $i \in A$, using (58), we have

$$
\begin{aligned}
S_{i}^{G} & =\alpha\left[\Pi_{k=1}^{K} Q\left(p^{k}, p^{i}, y^{k}, y^{i}\right)\right]^{1 / K} \\
& =\alpha\left[\Pi_{k \in A} Q\left(\alpha_{k} p, \alpha_{i} p, \beta_{k} y^{a}, \beta_{i} y^{a}\right) \Pi_{m \in B} Q\left(\gamma_{m} p^{b}, \alpha_{i} p^{a}, \delta_{m} y^{b}, \beta_{i} y^{a}\right)\right]^{1 / K} \\
& =\alpha \beta_{i}\left[\Pi_{k \in A} \beta_{k}^{-1} \Pi_{m \in B} \delta_{m}^{-1} Q\left(p^{b}, p^{a}, y^{b}, y^{a}\right)\right]^{1 / K} \quad \text { using BT3 and BT } \\
& =\beta_{i} \alpha\left[\Pi_{k \in A} \beta_{k}^{-1}\right]\left[\Pi_{m \in B} \delta_{m}^{-1}\right] Q\left(p^{b}, p^{a}, y^{b}, y^{a}\right)^{\# B / K}
\end{aligned}
$$

where $\# B$ is the number of countries in the set of countries $B$. For $j \in B$, we have

$$
\begin{aligned}
S_{j}^{G} & =\alpha\left[\Pi_{k=1}^{K} Q\left(p^{k}, p^{j}, y^{k}, y^{j}\right)\right]^{1 / K} \\
& \left.=\alpha\left[\Pi_{k \in A} Q\left(\alpha_{k} p^{a}, \gamma_{j} p^{b}, \beta_{k} y^{a}, \delta_{j} y^{b}\right) \Pi_{m \in B} Q\left(\gamma_{m} p^{b}, \gamma_{j} p^{b}, \gamma_{m} y^{b}, \delta_{j} y^{b}\right)\right]^{1 / K}\right] \\
& =\alpha \delta_{j}\left[\Pi_{i \in A} \beta_{k}^{-1} Q\left(p^{a}, p^{b}, y^{a}, y^{b}\right) \Pi_{m \in B^{\prime}} \delta_{m}^{-1}\right]^{1 / K} \quad \text { using BT3 and BT5-BT8 } \\
& =\delta_{j} \alpha\left[\Pi_{k \in A} \beta_{k}^{-1}\right]\left[\Pi_{m \in B} \delta_{m}^{-1}\right] Q\left(p^{a}, p^{b}, y^{a}, y^{b}\right)^{\# A / K}
\end{aligned}
$$

Using $\Sigma_{i \in A} \beta_{i}=1, \Sigma_{j \in B} \delta_{j}=1$, (A39) and (A40), we find

$$
\begin{array}{rlrl}
\Sigma_{j \in B} S_{j}^{G} / \Sigma_{i \in A} S_{i}^{G} & =Q\left(p^{a}, p^{b}, y^{a}, y^{b}\right)^{\# A / K} / Q\left(p^{b}, p^{a}, y^{b}, y^{a}\right)^{\# B / K} \\
& =Q\left(p^{a}, p^{b}, y^{a}, y^{b}\right)^{(\# A)+(\# B)] / K} & \text { using BT12 } \\
& =Q\left(p^{a}, p^{b}, y^{a}, y^{b}\right) & \text { since }(\# A)+(\# B)=K
\end{array}
$$

Exactness Properties of the Gini-EKS System: Using (58) with $Q=Q_{F}$, the system of functional equations (12) becomes: for $1 \leq i \neq j \leq K$ :

$$
\begin{aligned}
\Pi_{k=1}^{K} Q_{F}\left(\nabla f\left(y^{K}\right) e_{k}, \nabla f\left(y^{i}\right) e_{i}, y^{k}, y^{i}\right) / \Pi_{m=1}^{K} Q_{F}\left(\nabla f\left(y^{m}\right) e_{m},\right. & \left.\left.\nabla f\left(y^{j}\right) e_{j}, y^{m}, y^{j}\right)\right] \\
& =\left[f\left(y^{i}\right) / f\left(y^{j}\right)\right]^{K}
\end{aligned}
$$

If $f$ is the homogeneous quadratic defined by (41), then it is known (see Diewert [1976; 116] for references to the literature) that

$$
Q_{F}\left[\nabla f\left(y^{k}\right) e_{k}, \nabla f\left(y^{i}\right) e_{i}, y^{k}, y^{i}\right]=f\left(y^{i}\right) / f\left(y^{k}\right) \quad \text { for all } i \text { and } k .
$$

Substituting (A42) into (A41) leads to the identity

$$
\left[f\left(y^{i}\right) / f\left(y^{j}\right)\right]^{K}=\left[f\left(y^{i}\right) / f\left(y^{j}\right)\right]^{K} .
$$

Hence the Gini-EKS system is exact for the $f$ defined by (41) and hence is a superlative system. 
A similar proof shows that the unit cost function $c(p) \equiv\left(p^{T} B p\right)^{1 / 2}$ defined by (42) satisfies the system of functional equations (13) when the country shares are defined by (58) and $Q=Q_{F}$. The counterpart to (A42) that we require is

$$
Q_{F}\left[p^{k}, p^{i}, \nabla c\left(p^{k}\right) u_{k}, \nabla c\left(p^{i}\right) u_{i}\right]=u_{i} / u_{k} \text { for all } i \text { and } k \text {. }
$$

To establish (A43), use a result in Diewert $[1976 ; 133-134]$ with $r=2$.

\section{Proof of Proposition 9:}

T1: Using (67), BT1 and BT2, it is evident that $T 1$ is satisfied.

T2: Using BT3, BT5 and BT6 and substituting into (67), we obtain

$$
S^{i}=\alpha\left[\Sigma_{k=1}^{K}\left(\beta_{k} / \beta_{i}\right)\right]^{-1}=\alpha \beta_{i} ; \quad i=1, \ldots, K
$$

Thus $\alpha=1$ and $S^{i}=\beta_{i}$ as required.

T3: Using BT4, BT7 and BT8 and substituting into (67), we obtain

$S^{i}=\alpha\left[\Sigma_{k=1}^{K} p \cdot y^{k} / p \cdot y^{i}\right]^{-1}=p \cdot y^{i} \alpha /\left[\Sigma_{k=1}^{K} p \cdot y^{k}\right] ; \quad i=1, \ldots, K$.

Thus $S^{i}$ is proportional to $p \cdot y^{i}$ and $T 3$ is satisfied.

T4: Follows from (67) and BT10.

T5: Follows from (67) and BT9.

T6: Obvious from the symmetry of (67).

T7: Use BT7 and BT8 to establish this property.

T9: Using (67), BT3, BT12 and BT13, we see that if any component of $y^{i}$ increases, then $S^{i} / \alpha$ increases and the other $S^{j} / \alpha$ decrease. Hence $S^{i}$ will increase as any component of $y^{i}$ increases.

T10(i): Using (67), for $i \in A$, we have:

$$
\begin{aligned}
S^{i}= & \alpha\left[\Sigma_{k=1}^{K} Q\left(p^{k}, p^{i}, y^{k}, y^{i}\right)^{-1}\right]^{-1} \\
= & \alpha\left[\Sigma_{k \in A} Q\left(\alpha_{k} p^{a}, \alpha_{i} p^{a}, \beta_{k} y^{a}, \beta_{i} y^{a}\right)^{-1}+\Sigma_{k \in B} Q\left(p^{k}, \alpha_{i} p^{a}, y^{k}, \beta_{i} y^{a}\right)^{-1}\right]^{-1} \\
= & \alpha\left[\Sigma_{k \in A}\left(\beta_{k} / \beta_{i}\right) Q\left(p^{a}, p^{a}, y^{a}, y^{a}\right)^{-1}+\Sigma_{k \in b} \beta_{i}^{-1} Q\left(p^{k}, p^{a}, y^{k}, y^{a}\right)^{-1}\right]^{-1} \\
& \quad \text { using BT5-BT8 } \\
= & \beta_{i} \alpha\left[1+\Sigma_{k \in B} Q\left(p^{k}, p^{a}, y^{k}, y^{a}\right)^{-1}\right]^{-1} \\
& \quad \text { using BT3 and } \Sigma_{k \in A} \beta_{k}=1 .
\end{aligned}
$$


Therefore, for $i$ and $j$ belonging to $A$, we have $S^{i} / S^{j}=\beta_{i} / \beta_{j}$ which establishes part (i). To establish part (ii), let $i \in B$. Using (67):

$$
\begin{aligned}
S^{i} & =\alpha\left[\Sigma_{k=1}^{K} Q\left(p^{k}, p^{i}, y^{k}, y^{i}\right)^{-1}\right]^{-1} \\
& =\alpha\left[\Sigma_{k \in A} Q\left(\alpha_{k} p^{a}, p^{i}, \beta_{k} y^{a}, y^{i}\right)^{-1}+\Sigma_{k \in B} Q\left(p^{k}, p^{i}, y^{k}, y^{i}\right)^{-1}\right]^{-1} \\
& =\alpha\left[\Sigma_{k \in A} \beta_{k} Q\left(p^{a}, p^{i}, y^{a}, y^{i}\right)^{-1}+\Sigma_{k \in B} Q\left(p^{k}, p^{i}, y^{k}, y^{i}\right)^{-1}\right]^{-1} \quad \text { using } B T 6 \text { and } B T 8 \\
& =\alpha\left[Q\left(p^{a}, p^{i}, y^{a}, y^{i}\right)^{-1}+\Sigma_{k \in B} Q\left(p^{k}, p^{i}, y^{k}, y^{i}\right)^{-1}\right]^{-1} \quad \text { using } \Sigma_{k \in A} \beta_{k}=1 \\
& =\alpha S^{i *} .
\end{aligned}
$$

T11: Making the assumptions for $T 11$ and using (67), for $i \in A$, we have:

$$
\begin{aligned}
S^{i}= & \alpha\left[\Sigma_{k=1}^{K} Q\left(p^{k}, p^{i}, y^{k}, y^{i}\right)^{-1}\right]^{-1} \\
= & \alpha\left[\Sigma_{k \in A} Q\left(\alpha_{k} p^{a}, \alpha_{i} p^{a}, \beta_{k} y^{a}, \beta_{i} y^{a}\right)^{-1}+\Sigma_{k \in B} Q\left(\gamma_{k} p^{b}, \alpha_{i} p^{a}, \delta_{k} y^{b}, \beta_{i} y^{a}\right)^{-1}\right]^{-1} \\
= & \alpha\left[\Sigma_{k \in A}\left(\beta_{k} / \beta_{i}\right) Q\left(p^{a}, p^{a}, y^{a}, y^{a}\right)^{-1}+\Sigma_{k \in B}\left(\delta_{k} / \beta_{i}\right) Q\left(p^{b}, p^{a}, y^{b}, y^{a}\right)^{-1}\right]^{-1} \\
& \quad \text { using BT5-BT8 } \\
= & \beta_{i} \alpha\left[1+Q\left(p^{b}, p^{a}, y^{b}, y^{a}\right)^{-1}\right]^{-1} \quad \text { using BT3, } \Sigma_{k \in A} \beta_{k}=1 \text { and } \Sigma_{k \in B} \delta_{k}=1 .
\end{aligned}
$$

Similarly, for $j \in B$, we have

$$
\begin{aligned}
S^{j} & =\alpha\left[\Sigma_{k=1}^{K} Q\left(p^{k}, p^{j}, y^{k}, y^{j}\right)^{-1}\right]^{-1} \\
& =\alpha\left[\Sigma_{k \in A} Q\left(\alpha_{k} p^{a}, \gamma_{j} p^{b}, \beta_{k} y^{a}, \delta_{j} y^{b}\right)^{-1}+\Sigma_{k \in B} Q\left(\gamma_{k} p^{b}, \gamma_{j} p^{b}, \delta_{k} y^{b}, \delta_{j} y^{b}\right)^{-1}\right]^{-1} \\
& =\alpha\left[\Sigma_{k \in A}\left(\beta_{k} / \delta_{j}\right) Q\left(p^{a}, p^{b}, y^{a}, y^{b}\right)^{-1}+\Sigma_{k \in B}\left(\delta_{k} / \delta_{j}\right) Q\left(p^{b}, p^{b}, y^{b}, y^{b}\right)^{-1}\right]^{-1} \\
& =\delta_{j} \alpha\left[Q\left(p^{a}, p^{b}, y^{a}, y^{b}\right)^{-1}+1\right]^{-1} .
\end{aligned}
$$

Using (A44), (A45), $\Sigma_{i \in A} \beta_{i}=1$ and $\Sigma_{j \in B} \delta_{j}=1$, we have

$$
\begin{aligned}
\Sigma_{j \in B} S^{j} & (P, Y) / \Sigma_{i \in A} S^{i}(P, Y) \\
& =\left[1+Q\left(p^{b}, p^{a}, y^{b}, y^{a}\right)^{-1}\right] /\left[1+Q\left(p^{a}, p^{b}, y^{a}, y^{b}\right)^{-1}\right] \\
& =\left[1+Q\left(p^{a}, p^{b}, y^{a}, y^{b}\right)\right] /\left[1+Q\left(p^{a}, p^{b}, y^{a}, y^{b}\right)^{-1}\right] \text { using BT11 } \\
& =Q\left(p^{a}, p^{b}, y^{a}, y^{b}\right)
\end{aligned}
$$

since $(1+\beta) /\left(1+\beta^{-1}\right)=\beta$ where $\beta \equiv Q\left(p^{a}, p^{b}, y^{a}, y^{b}\right)$.

Exactness Properties of the Fisher Oun Share System: Using (67) with $Q=Q_{F}$, the system of functional equations (12) becomes: for $1 \leq i \neq j \leq K$ :

$$
\begin{aligned}
& {\left[\Sigma_{k=1}^{K} Q_{F}\left(\nabla f\left(y^{k}\right) e_{k}, \nabla f\left(y^{i}\right) e_{i}, y^{k}, y^{i}\right)^{-1}\right]^{-1}} \\
& \quad /\left[\Sigma_{m=1}^{K} Q_{F}\left(\nabla f\left(y^{m}\right) e_{m}, \nabla f\left(y^{j}\right) e_{j}, y^{m}, y^{j}\right)^{-1}\right]^{-1}=f\left(y^{i}\right) / f\left(y^{j}\right) .
\end{aligned}
$$


Substituting (A42) into the above equations leads to the following system of equations: for $1 \leq i \neq j \leq K$ :

$$
\left[\Sigma_{k=1}^{K} f\left(y^{k}\right) / f\left(y^{i}\right)\right]^{-1} /\left[\Sigma_{m=1}^{K} f\left(y^{m}\right) / f\left(y^{j}\right)\right]^{-1}=f\left(y^{i}\right) / f\left(y^{j}\right)
$$

which is a system of identities. Hence the $f$ defined by (41) is exact for the Gini-EKS system.

Turning now to the system of functional equations (13), substituting (67) into these equations with $Q \equiv Q_{F}$ leads to the following system of equations for $1 \leq i \neq j \leq K$ :

$$
\begin{aligned}
& {\left[\Sigma_{k=1}^{K} Q_{F}\left(p^{k}, p^{i}, \nabla c\left(p^{i}\right) u_{k}, \nabla c\left(p^{i}\right) u_{i}\right)^{-1}\right]^{-1} /} \\
& \quad\left[\Sigma_{m=1}^{K} Q_{F}\left(p^{m}, p^{j}, \nabla c\left(p^{m}\right) u_{m}, \nabla c\left(p^{j}\right) u_{j}\right)^{-1}\right]^{-1}=u_{i} / u_{j} .
\end{aligned}
$$

Substituting (A43) into the above equations leads to the system of identities $\left[\Sigma_{k=1}^{K} u_{k} / u_{i}\right]^{-1} /\left[\Sigma_{m=1}^{K} u_{m} / u_{j}\right]^{-1}=u_{i} / u_{j}$.

Hence the $c$ defined by (42) is exact for the Gini-EKS system.

Proof of Proposition 10:

T1: The proof of existence and continuity of the share functions is somewhat involved. Consider the minimization problem (71). If we set $S_{K}=1$ and solve the resulting minimization problem in $S_{1}, \ldots, S_{K-1}$, we can normalize the solution to satisfy (73). Denote the objective function in (71) with $S_{K}=1$ by $f\left(S_{1}, \ldots, S_{K-1}\right)$. Denote $Q\left(p^{j}, p^{k}, y^{j}, y^{k}\right)$ by $Q_{j k}$ for $1 \leq j, k \leq K$. Note that BT1 implies $Q_{j k}>0$. The first order necessary conditions for our $S_{K}=1$ modification of (71) are:

$$
\Sigma_{j=1}^{K-1}\left[Q_{i j} / S_{j}^{*}\right]-\Sigma_{k=1}^{K-1}\left[Q_{k i} S_{k}^{*} / S_{i}^{* 2}\right]-Q_{K i} / S_{i}^{* 2}=-Q_{i k} ; \quad i=1, \ldots, K-1 .
$$

The arguments of Van Yzeren [1956; 25-26] can be adapted to show that a unique positive $S_{1}^{*}, \ldots, S_{K-1}^{*}$ solution to (A46) exists. We now show that the matrix of second order partial derivatives of $f$ evaluated at the solution, $\nabla^{2} f\left(S_{1}^{*}, \ldots, S_{K-1}^{*}\right) \equiv\left[f_{i j}\left(S_{1}^{*}, \ldots, S_{K-1}^{*}\right)\right]$, is positive definite. Differentiating the left hand side of (A46) with respect to $S_{j}$, we obtain the following expressions for the second order partial derivatives of $f$ :

$$
\begin{aligned}
& f_{i i}\left(S_{1}, \ldots, S_{K-1}\right)=2 \Sigma_{k=1, k \neq i}^{K-1}\left[Q_{k i} S_{k} / S_{i}^{3}\right]+2 Q_{K i} / S_{i}^{3} ; \quad i=1, \ldots, K-1 \\
& f_{i j}\left(S_{1}, \ldots, S_{K-1}\right)=-\left[Q_{i j} / S_{j}^{2}\right]-\left[Q_{j i} / S_{i}^{2}\right] ; \quad 1 \leq i \neq j \leq K-1 .
\end{aligned}
$$


Use the $i$ th equation in (A46) to solve for $\Sigma_{k=1, k \neq i}^{K-1} Q_{k i} S_{k}^{*} / S_{i}^{* 2}$ and substitute the resulting expression into the right hand side of (A47). Using the resulting equation and equations (A48) evaluated at $S_{1}^{*}, \ldots, S_{K-1}^{*}$, we find that

$$
\Sigma_{j=1}^{K-1} f_{i j}\left(S_{1}^{*}, \ldots, S_{K}^{*}\right) S_{j}^{*}=Q_{i K}+Q_{K i} / S_{i}^{* 2}>0 ; \quad i=1, \ldots, K-1,
$$

where the inequalities in (A49) follow from the positivity of the $Q_{i j}$. The positivity of the $Q_{i j}$ also implies via (A47) that $f_{i i}\left(S_{1}^{*}, \ldots, S_{K-1}^{*}\right)>0$ for $i=1, \ldots, K-1$ and that $f_{i j}\left(S_{1}^{*}, \ldots, S_{K-1}^{*}\right)<0$ for $1 \leq i \neq j \leq K-1$. Since the $S_{j}^{*}$ are all positive, the inequalities (A49) imply that the matrix $\nabla^{2} f\left(S_{1}^{*}, \ldots, S_{K-1}^{*}\right)$ has dominant diagonal (see Gale and Nikaido $[1965 ; 84]$ for a definition). Note also that $\nabla^{2} f\left(S_{1}^{*}, \ldots, S_{K-1}^{*}\right)$ has positive main diagonal elements and negative off diagonal elements and hence is what Gale and Nikaido $[1965 ; 86]$ call a Leontief type matrix. Thus the matrix $\nabla^{2} f\left(S_{1}^{*}, \ldots, S_{K-1}^{*}\right)$ is a dominant diagonal Leontief type matrix and by result noted by Gale and Nikaido [1965; 86], this matrix is a $P$-matrix; i.e., all of its principle submatrices have positive determinants. In particular, the determinant of $\nabla^{2} f\left(S_{1}^{*}, \ldots, S_{K-1}^{*}\right)$ is positive and hence the inverse matrix $\left[\nabla^{2} f\left(S_{1}^{*}, \ldots, S_{K-1}^{*}\right)\right]^{-1}$ exists. (For later reference, by another result in Gale and Nikaido $[1965 ; 86]$, all of the elements in this inverse matrix are positive). Since the $Q_{i j} \equiv Q\left(p^{i}, p^{j}, y^{i}, y^{j}\right)$ are once continuously differentiable functions of their arguments by assumption and using the fact that $\left[\nabla^{2} f\left(S_{1}^{*}, \ldots, S_{K-1}^{*}\right)\right]^{-1}$ exists, we can apply the Implicit Function Theorem (see Rudin [1953; 177-182]) to the system of equations (A46) to obtain the continuity (and once continuous differentiability) of the solution functions $S_{i}^{*}(P, Y)$ with respect to the elements of the matrices $P$ and $Y$.

T2: Substituting the assumptions of the test into (72) and using BT3, BT5 and BT6 yields the following system of equations:

$$
\Sigma_{j=1}^{K}\left(\beta_{j} / \beta_{i}\right)\left(S_{i} / S_{j}\right)=\Sigma_{k=1}^{K}\left(\beta_{i} / \beta_{k}\right)\left(S_{k} / S_{i}\right) ; \quad i=1, \ldots, K
$$

Obviously, the unique solution to this system of equations which also satisfies the normalization (73) is $S_{k}=\beta_{k}$ for $k=1, \ldots, K$.

T3: Substituting the assumptions of the test into (72) and using BT4, BT7 and BT8 yields the following system of equations:

$$
\Sigma_{j=1}^{K}\left(p \cdot y^{j} / p \cdot y^{i}\right)\left(S_{i} / S_{j}\right)=\Sigma_{k=1}^{K}\left(p \cdot y^{i} / p \cdot y^{k}\right)\left(S_{k} / S_{i}\right) ; \quad i=1, \ldots, K .
$$

Obviously, the solution ray to the above system of equations is $S_{k}=\alpha p \cdot y^{k}, k=1, \ldots, K, \alpha>$ 0 . Using the normalization (73) picks a unique point on this solution ray and demonstrates that test $T 3$ is satisfied. 
T4: This test follows from (72) and BT10.

T5: This test follows from (72) and BT9.

T6: This test follows from the symmetric nature of equations (72) and (73).

T7: This test follows using BT7 and BT8.

T8: Let $S_{1}^{*}, \ldots, S_{K}^{*}$ be the solution to (72) and (73) when we have price vectors $p^{k}$ and quantity vectors $y^{k}$ for $k=1, \ldots, K$. For $\lambda>0$, change $y^{1}$ into $\lambda y^{1}$. Using BT5 and BT6, it is easy to show that $\lambda S_{1}^{*}, S_{2}^{*}, \ldots, S_{K}^{*}$ will satisfy equations (72) with $y^{1}$ replaced everywhere by $\lambda y^{1}$ (the $\lambda$ factors cancel out, leaving the original system of equations). An analogous property holds if $y^{2}$ is replaced by $\lambda y^{2}$, etc.

T9: Consider the minimization problem (71) when we set $S_{K}=1$. Denote the remaining shares as $S_{1}^{*}(P, Y), \ldots, S_{K-1}^{*}(P, Y)$. Using the results established in the proof of $T 1$ and differentiating equations (A46) with respect to the components of $y^{K}$, we obtain the following formula for the derivatives of the $S_{i}^{*}$ with respect to the components of $y^{K}$ for $i=1,2, \ldots, K-1$ :

$$
\begin{aligned}
& \nabla_{y^{K}} S_{i}^{*}(P, Y) \\
& \begin{aligned}
=\quad \Sigma_{j=1}^{K-1} e_{i}^{T}\left[\nabla^{2} f\left(S_{1}^{*}, \ldots, S_{K-1}^{*}\right)\right]^{-1} e_{j}\left[-\nabla_{y^{K}} Q\left(p^{j}, p^{K}, y^{j}, y^{K}\right)\right. \\
\left.\quad+\left(S_{j}^{*}\right)^{-2} \nabla_{y^{K}} Q\left(p^{K}, p^{j}, y^{K}, y^{j}\right)\right]
\end{aligned}
\end{aligned}
$$

where $e_{i}$ is the $i$ th unit vector of dimension $K-1$. From the proof of $T 1$, the $K-1$ by $K-1$ matrix $\left[\nabla^{2} f\left(S_{1}^{*}, \ldots, S_{K-1}^{*}\right)\right]^{-1}$ has all elements positive. Using BT12, the vector of derivatives $\nabla_{y^{K}} Q\left(p^{i}, p^{K}, y^{i}, y^{K}\right)$ is nonnegative and positive almost everywhere. Using BT13, the vector of derivatives $\nabla_{y^{K}} Q\left(p^{K}, p^{i}, y^{K}, y^{i}\right)$ is nonpositive and negative almost everywhere. Hence the vector of derivatives $\nabla_{y^{K}} S_{i}^{*}(P, Y)$ is nonpositive and negative almost everywhere. Thus $S_{i}^{*}(P, Y)$ is decreasing in the components of $y^{K}$ for $i=1,2, \ldots, K-1$. Switching now to the model that uses the normalization (73), we see that the above results imply that $S_{K}(P, Y)$ is increasing in the components of $y^{K}$. Using the symmetry of equations (72) and (73), this suffices to establish that each $S_{k}(P, Y)$ is increasing in the components of $y^{k}$ for $k=1, \ldots, K$.

T10(i): Under the assumptions for the test, for $i \in A$, equations (72) become:

$$
\begin{aligned}
& \Sigma_{k \in A}\left(\beta_{k} / \beta_{i}\right)\left(S_{i} / S_{k}\right)+\Sigma_{k \in B} \beta_{i}^{-1} Q\left(p^{a}, p^{k}, y^{a}, y^{k}\right) S_{i} / S_{k} \\
& \quad=\Sigma_{k \in A}\left(\beta_{k} / \beta_{k}\right) S_{k} / S_{i}+\Sigma_{k \in B} \beta_{i} Q\left(p^{k}, p^{a}, y^{k}, y^{a}\right) S_{k} / S_{i}
\end{aligned}
$$


where we have used BT3 and BT5-BT8. For $k \in A$, set $S_{k}=\beta_{k} S_{a}$. Then the above equations become for $i \in A$.

$$
(\# A)+\Sigma_{k \in B} Q\left(p^{a}, p^{k}, y^{a}, y^{k}\right) S_{a} / S_{k}=(\# A)+\Sigma_{k \in B} Q\left(p^{k}, p^{a}, y^{k}, y^{a}\right) S_{k} / S_{a} .
$$

Note that equations (A50) do not depend on $i$. Thus there is only one independent equation in (A50). For $j \in B$, equations (72) become, (assuming $S_{k}=\beta_{k} S_{a}$ for $k \in A$ ):

$$
\begin{aligned}
& (\# A) Q\left(p^{j}, p^{a}, y^{j}, y^{a}\right) S_{j} / S_{a}+\Sigma_{k \in B} Q\left(p^{j}, p^{k}, y^{j}, y^{k}\right) S_{j} / S_{k} \\
& \quad=(\# A) Q\left(p^{a}, p^{j}, y^{a}, y^{j}\right) S_{a} / S_{j}+\Sigma_{k \in B} Q\left(p^{k}, p^{j}, y^{k}, y^{j}\right) S_{k} / S_{j} .
\end{aligned}
$$

Equation (A50) and equations (A51) for $j \in B$ along with the normalizing equation $S_{a}+\Sigma_{k \in B} S_{k}=1$ can be solved for $S_{a}$ and $S_{j}$ for $j \in B$. Once $S_{a}$ has been determined, we have $S_{i} \equiv \beta_{i} S_{a}$ for $i \in A$ and part (i) of $T 10$ holds. However, equations (A51) show that part (ii) of $T 10$ does not hold; note the factor $\# A \equiv$ the number of countries in the subbloc $A$.

T11: Substitute the assumptions of test $T 11$ into equations (72). For $i \in A$, let $S_{i}=\beta_{i} S_{a}$ and for $j \in B$, let $S_{j}=\delta_{j} S_{b}$. For $i \in A$, each of these equations in (72) reduces to

$$
(\# A)+(\# B) Q\left(p^{a}, p^{b}, y^{a}, y^{b}\right) S_{a} / S_{b}=(\# A)+(\# B) Q\left(p^{b}, p^{a}, y^{b}, y^{a}\right) S_{b} / S_{a}
$$

where we have used BT3 and BT5-BT8. For $j \in B$, each of these equations in (72) reduces to

$$
(\# A) Q\left(p^{b}, p^{a}, y^{b}, y^{a}\right) S_{b} / S_{a}+(\# B)=(\# A) Q\left(p^{a}, p^{b}, y^{a}, y^{b}\right) S_{a} / S_{b}+(\# B) .
$$

Both of the equations (A52) and (A53) simplify to

$$
\begin{aligned}
\Sigma_{j \in B} S_{j} / \Sigma_{i \in A} S_{i}=S_{b} / S_{a} & =\left[Q\left(p^{a}, p^{b}, y^{a}, y^{b}\right) / Q\left(p^{b}, p^{a}, y^{b}, y^{a}\right)\right]^{1 / 2} \\
& =Q\left(p^{a}, p^{b}, y^{a}, y^{b}\right)
\end{aligned}
$$

where (A54) follows from the line above if $Q$ satisfies the bilateral time reversal test $B T 11$. Note that this is the only part of the proof where test $B T 11$ is used. If $Q$ is equal to either the Paasche $Q_{P}$ or Laspeyres $Q_{L}$ quantity index, then it can be verified that these two indexes satisfy all of the bilateral tests except BT11. However, if either $Q=Q_{L}$ or $Q=Q_{P}$ is inserted into (A54), we find that $S_{b} / S_{a}=Q_{F}\left(p^{a}, p^{b}, y^{a}, y^{b}\right)$, the Fisher ideal index. Thus if $Q=Q_{L}$ or $Q_{P}$, all multilateral tests except $T 10$ and $T 12$ are satisfied.

Exactness Properties of the Unweighted Balanced Method: For $Q=Q_{F}$, substituting (10), (12) and (A42) into (72) leads to the following equations for $i=1, \ldots, K$ : 
$\Sigma_{j=1}^{K}\left[f\left(y^{j}\right) / f\left(y^{i}\right)\right]\left[f\left(y^{i}\right) / f\left(y^{j}\right)\right]=\Sigma_{k=1}^{K}\left[f\left(y^{i}\right) / f\left(y^{k}\right)\right]\left[f\left(y^{k}\right) / f\left(y^{i}\right)\right]$

which is a system of identities. Hence the homogeneous quadratic $f$ defined by (41) is exact for this method. Similarly, for $Q=Q_{F}$, substituting (9), (13) and (A42) into (72) leads to the following equations for $i=1, \ldots, K$ :

$\Sigma_{j=1}^{K}\left[u_{j} / u_{i}\right]\left[u_{i} / u_{j}\right]=\Sigma_{k=1}^{K}\left[u_{i} / u_{k}\right]\left[u_{k} / u_{i}\right]$

which is a system of identities. Hence the homogeneous quadratic unit cost function defined by (42) is also exact for the unweighted balanced method when $Q=Q_{F}$.

For $Q=Q_{L}\left(p^{i}, p^{j}, y^{i}, y^{j}\right) \equiv p^{i} \cdot y^{j} / p^{i} \cdot y^{i}$, the Laspeyres quantity index, equations (72) become:

$$
\Sigma_{j=1}^{K}\left(p^{i} \cdot y^{j} / p^{i} \cdot y^{i}\right)\left(S_{i} / S_{j}\right)=\Sigma_{k=1}^{K}\left(p^{k} \cdot y^{i} / p^{k} \cdot y^{k}\right)\left(S_{k} / S_{i}\right) ; \quad i=1, \ldots, K
$$

Substituting (10) and (12) into (A55) and letting $f$ be defined by (41) leads to the following system of equations:

$$
\Sigma_{j=1}^{K}\left[y^{i T} A y^{j} / f\left(y^{i}\right) f\left(y^{j}\right)\right]=\Sigma_{k=1}^{K}\left[y^{k T} A y^{i} / f\left(y^{i}\right) f\left(y^{k}\right)\right] ; \quad i=1, \ldots, K
$$

where we have used $\nabla f\left(y^{i}\right)=A y^{i} / f\left(y^{i}\right)$. Since $A=A^{T}$, it can be verified that (A56) is a system of identities.

Substituting (9) and (13) into (A55) and letting $c$ be defined by (42) and using $\nabla c\left(p^{i}\right)=B p^{i} / c\left(p^{i}\right)$ leads to:

$$
\Sigma_{j=1}^{K}\left[p^{i T} B p^{j} / c\left(p^{i}\right) c\left(p^{j}\right)\right]=\Sigma_{k=1}^{K}\left[p^{k T} B p^{i} / c\left(p^{i}\right) / c\left(p^{k}\right)\right] ; \quad i=1, \ldots, K
$$

Using $B=B^{T}$, it can be verified that (A57) is a system of identities.

The use of $Q=Q_{P}$ in (72) where $\left.Q_{P}\left(p^{i}, p^{j}, y^{i}, y^{j}\right) \equiv p^{j} \cdot y^{j} / p^{j} \cdot y^{i}\right)$ corresponds to Gerardi's [1974] version of the unweighted balanced method; see also Van Ijzeren [1983; 45-46]. Hence the identities (A56) and (A57) show that this version of the unweighted balanced method is exact for the homogeneous quadratic aggregator function defined by (41) and is also exact for the homogeneous quadratic unit cost function defined by (42). Hence when $Q=Q_{P}$, the unweighted balanced method is superlative.

Suppose now that $Q=Q_{L}$ where $Q_{L}\left(p^{i}, p^{j}, y^{i}, y^{j}\right) \equiv p^{i} \cdot y^{j} / p^{i} \cdot y^{i}$ is the Laspeyres bilateral quantity index. This corresponds to Van Yzeren's $[1956 ; 15-20]$ original unweighted balanced method; see also Van Ijzeren [1983; 44-45] [1987; 59-61]. In a manner similar to our derivation of equations (A55) - (A57) above, we can show that the homogeneous quadratic $f$ and $c$ defined by (41) and (42) are also exact for this $Q=Q_{L}$ version of the unweighted balanced method. Hence Van Yzeren's original unweighted balanced method is also superlative. 
Proof of Proposition 11:

T1: We have already established the existence and positivity of the $S_{i}$ using only $B T 1$. It remains to establish the continuity of the $S_{\mathrm{i}}(P, Y)$. Using (79), BT1 and BT2, the elements in the matrix $A$ will be continuous functions of the elements in the matrices $P$ and $Y$. Using a Theorem of Frobenius [1908; 473], the determinant $\left|I_{K-1}-\tilde{A}\right|>0$ and so the $\tilde{x}$ defined by (83) will be continuous in the elements of $A$. Thus using $x_{N}=1$ and (82), the continuity of the $S_{i}(P, Y)$ in the elements of $P$ and $Y$ follows.

T2: Substituting the conditions of the test into (78) and using BT3, BT5 and BT6 yields the following system of equations:

$$
\Sigma_{j=1}^{K}\left(\beta_{j} / \beta_{i}\right) S_{i}^{2}=\Sigma_{k=1}^{K}\left(\beta_{i} / \beta_{k}\right) S_{k}^{2} ; \quad i=1, \ldots, K
$$

Substituting $S_{i}=\beta_{i}$ into the above equations yields a system of identities.

T3: Substituting the conditions of the test into (78) and using BT4, BT7 and BT8 yields:

$$
\Sigma_{j=1}^{K}\left(p \cdot y^{j} / p \cdot y^{i}\right) S_{i}^{2}=\Sigma_{k=1}^{K}\left(p \cdot y^{i} / p \cdot y^{k}\right) S_{k}^{2} ; \quad i=1, \ldots, K .
$$

Setting $S_{i}=\alpha p \cdot y^{i}$ for $i=1, \ldots, K$ solves the above equations.

T4: This test follows using equations (78) and BT10.

T5: This test follows using (78) and BT9.

T6: This test follows from the symmetric nature of equations (73) and (78).

T7: This test follows using BT7 and BT8.

T8: This test fails in general unless the bilateral quantity index $Q$ satisfies circularity. But Proposition 7 shows that circularity is not consistent with the satisfaction of tests BT1-BT13. Thus under our hypotheses on $Q$, test $T 8$ fails.

T9: By the symmetry of the method, we need only set $x_{N}=1$ and show that the $x_{1}, \ldots, x_{N-1}$ that satisfy (83) are decreasing functions of the components of the country $K$ quantity vector $y^{K}$. Define the $j$ th column of the $A$ matrix with row $K$ deleted by $\tilde{A}_{\text {.j }}$ for $j=1,2, \ldots, K$. Note that $\tilde{A}_{\bullet K}=\tilde{a}$ where $\tilde{a}$ appears in (83). Differentiating equations (83) with respect to the elements of $y^{K}$ yields the following formula for the $K-1$ by $N$ matrix of derivatives of the elements of $\tilde{x}$ with respect to the elements of $y^{K}$ :

$$
\nabla_{y} \tilde{x}^{\tilde{x}}=\left[I_{K-1}-\tilde{A}\right]^{-1}\left\{\nabla_{y^{K}} \tilde{A}_{\bullet K}+\Sigma_{j=1}^{K-1}\left(\nabla_{y^{K}} \tilde{A}_{\bullet j}\right) x_{j}\right\} .
$$


From (84), the elements of $\left[I_{K-1}-\tilde{A}\right]^{-1}$ are all positive. Differentiating the elements of the $A$ matrix using definitions (79) and the monotonicity properties of $Q, \mathrm{BT} 12$ and BT13, the matrices of derivatives $\nabla_{y^{K}} \tilde{A}_{\cdot j}$ are nonpositive and negative almost everywhere for $j=1, \ldots, K$. Using these facts plus the positivity of the $x_{j}$, (A58) implies that $\nabla_{y} K \tilde{x}$ is nonpositive and negative almost everywhere. Thus the $x_{i}(P, Y)$ for $i=1, \ldots, K-1$ are decreasing in the components of $y^{K}$.

T10: Let $p^{a}>>0_{N}, y^{a}>>0_{N}, \alpha_{i}>0, \beta_{i}>0, p^{i}=\alpha_{i} p^{a}, y^{i}=\beta_{i} y^{a}$ for $i \in A$ with $\Sigma_{i \in A} \beta_{i}=1$. For $i \in A$, equations (78) become, using BT3 and BT5-BT8:

$$
\begin{aligned}
\Sigma_{j \in A}\left(\beta_{j} / \beta_{i}\right) S_{i}^{2}+ & \Sigma_{j \in B} \beta_{i}^{-1} Q\left(p^{a}, p^{j}, y^{a}, y^{j}\right) S_{i}^{2} \\
& =\Sigma_{k \in A}\left(\beta_{i} / \beta_{k}\right) S_{k}^{2}+\Sigma_{k \in B} \beta_{i} Q\left(p^{k}, p^{a}, y^{k}, y^{a}\right) S_{k}^{2} .
\end{aligned}
$$

For $k \in A$, let $S_{k}=\beta_{k} S_{a}$. Using $\Sigma_{j \in A} \beta_{j}=1$ and BT3, the above equations become for $i \in A$ :

$$
\begin{aligned}
Q\left(p^{a}, p^{a}, y^{a}, y^{a}\right) S_{a}^{2}+ & \Sigma_{j \in B} Q\left(p^{a}, p^{j}, y^{a}, y^{j}\right) S_{a}^{2} \\
& =Q\left(p^{a}, p^{a}, y^{a}, y^{a}\right) S_{a}^{2}+\Sigma_{k \in B} Q\left(p^{k}, p^{a}, y^{k}, y^{a}\right) S_{k}^{2} .
\end{aligned}
$$

Note that equations (A59) do not depend on $i$ so there is only one independent equation in (A59). For $i \in B$, equations (78) become, using BT5-BT8 and $S_{k}=\beta_{k} S_{a}$ for $k \in A$ :

$$
\begin{aligned}
Q\left(p^{i}, p^{a}, y^{i}, y^{a}\right) S_{i}^{2} & +\Sigma_{j \in B} Q\left(p^{i}, p^{j}, y^{i}, y^{j}\right) S_{i}^{2} \\
& =Q\left(p^{a}, p^{i}, y^{a}, y^{i}\right) S_{a}^{2}+\Sigma_{k \in B} Q\left(p^{k}, p^{i}, y^{k}, y^{i}\right) S_{k}^{2}
\end{aligned}
$$

Equations (A59) and (A60) along with the equation $S_{a}+\Sigma_{k \in B} S_{k}=1$ can be solved for positive $S_{a}$ and $S_{k}$ for $k \in B$. Once $S_{a}$ has been determined, we set $S_{i}=\beta_{i} S_{a}$ for $i \in A$ and part (i) of $T 10$ holds. Examination of (A59) and (A60) shows that part (ii) also holds.

T11: Substitute the assumptions of test 11 into equations (78). For $i \in A$, let $S_{i}=\beta_{i} S_{a}$ and for $j \in B$, let $S_{j}=\delta_{j} S_{b}$. For $i \in A$, using BT3 and BT5-BT8, each of these equations in $(78)$ reduces to

$$
S_{a}^{2}+Q\left(p^{a}, p^{b}, y^{a}, y^{b}\right) S_{a}^{2}=S_{a}^{a}+Q\left(p^{b}, p^{a}, y^{b}, y^{a}\right) S_{b}^{2} .
$$

For $i \in B$, each of these equations in (78) reduces to

$$
Q\left(p^{b}, p^{a}, y^{b}, y^{a}\right) S_{b}^{2}+S_{b}^{2}=Q\left(p^{a}, p^{b}, y^{a}, y^{b}\right) S_{a}^{2}+S_{b}^{2} .
$$

Each of the equations (A61) and (A62) simplifies to 


$$
\begin{aligned}
\Sigma_{j \in B} S_{j} / \Sigma_{i \in A} S_{i} & =S_{b} / S_{a} \\
& =\left[Q\left(p^{a}, p^{b}, y^{a}, y^{b}\right) / Q\left(p^{b}, p^{a}, y^{b}, y^{a}\right)\right]^{1 / 2} \\
& =Q\left(p^{a}, p^{b}, y^{a}, y^{b}\right)
\end{aligned}
$$

where (A64) follows from (A63) if $Q$ satisfies the time reversal test BT11. This is the only place in the proof of Proposition 11 where we use property BT11. Using (A63), if $Q=Q_{L}$ or $Q=Q_{P}$, we find that $S_{b} / S_{a}=Q_{F}\left(p^{a}, p^{b}, y^{a}, y^{b}\right)$. As in the proof of Proposition 10, we note that $Q_{L}$ and $Q_{P}$ satisfy all of the bilateral tests except BT11. Hence if $Q=Q_{L}$ or $Q=Q_{P}$, then the resulting weighted balanced methods satisfy all of the multilateral tests except T8 and T12.

Exactness Properties of the Weighted Balanced Method: For $Q=Q_{F}$, substituting (10), (12) and (A42) into (78) leads to the following system of equations for $i=1, \ldots, K$ :

$$
\begin{aligned}
\Sigma_{j=1}^{K}\left[f\left(y^{j}\right) / f\left(y^{i}\right)\right] & =\Sigma_{k=1}^{K}\left[f\left(y^{i}\right) / f\left(y^{k}\right)\right]\left[f\left(y^{k}\right) / f\left(y^{i}\right)\right]^{2} \\
& =\Sigma_{k=1}^{K}\left[f\left(y^{k}\right) / f\left(y^{i}\right)\right]
\end{aligned}
$$

which is a system of identities. Hence the homogeneous quadratic $f$ defined by (41) is exact for this method. Similarly, for $Q=Q_{F}$, substituting (9), (13) and (A43) into (78) leads to the following system of equations for $i=1, \ldots, K$ :

$$
\begin{aligned}
\Sigma_{j=1}^{K}\left[u_{j} / u_{i}\right] & =\Sigma_{k=1}^{K}\left[u_{i} / u_{k}\right]\left[u_{k} / u_{i}\right]^{2} \\
& =\Sigma_{k=1}^{K}\left[u_{k} / u_{i}\right]
\end{aligned}
$$

which is a system of identities. Hence the homogeneous quadratic unit cost function $c$ defined by (42) is also exact for this method.

For $Q=Q_{L}$, the Laspeyres quantity index, equations (78) become:

$$
\Sigma_{j=1}^{K}\left[p^{i} \cdot y^{j} / p^{i} \cdot y^{i}\right]=\Sigma_{k=1}^{K}\left[p^{k} \cdot y^{i} / p^{k} \cdot y^{k}\right]\left[S_{k} / S_{i}\right]^{2} ; \quad i=1, \ldots, K .
$$

Substituting (10) and (12) into (A65) and letting $f$ be defined by (41) leads to the following system:

$$
\Sigma_{j=1}^{K}\left[y^{i T} A y^{j} / f\left(y^{i}\right)^{2}\right]=\Sigma_{k=1}^{K}\left[y^{k T} A y^{i} / f\left(y^{i}\right)^{2}\right] ; \quad i=1, \ldots, K
$$

which is a system of identities using $A=A^{T}$. Hence the homogeneous quadratic $f$ defined by (41) is exact for the weighted balanced method with $Q=Q_{L}$. In a similar fashion, we can show that the $c$ defined by (42) is exact for the weighted balanced method with $Q=Q_{L}$.

Finally, in an analogous fashion, it can be shown that the $f$ defined by (41) and the $c$ defined by (42) are exact for the weighted balanced method with $Q=Q_{P}$. 


\section{Appendix 2: A Simple Numerical Example}

We consider the simplest possible example of a multilateral method where there are 3 countries $(K=3)$ and 2 commodities $(N=2)$. As usual, let $p^{k}$ and $y^{k}$ denote the price and quantity vectors for country $k$. These 6 vectors are defined below:

$$
\begin{aligned}
& p^{1} \equiv\left(p_{1}^{1}, p_{2}^{1}\right) \equiv(1,1) ; p^{2} \equiv\left(p_{1}^{2}, p_{2}^{2}\right) \equiv(10, .1) ; p^{3} \equiv\left(p_{1}^{3}, p_{2}^{3}\right) \equiv(.1,10) \\
& y^{1} \equiv\left(y_{1}^{1}, y_{2}^{1}\right) \equiv(1,2) ; y^{2} \equiv\left(y_{1}^{2}, y_{2}^{2}\right) \equiv(1,100) ; y^{3} \equiv\left(y_{1}^{3}, y_{2}^{3}\right) \equiv(1000,10)
\end{aligned}
$$

Note that the geometric mean of the two prices in each country is unity across all countries; however, the structure of relative prices (and relative quantities) differs vastly across the three countries.

Nominal expenditures (expressed in a common currency) in the three countries are: $p^{1} \cdot y^{1} \equiv \Sigma_{n=1}^{2} p_{n}^{1} y_{n}^{1}=3 ; p^{2} \cdot y^{2}=20$ and $p^{3} \cdot y^{3}=200$. Thus country 1 is tiny, country 2 is medium sized and country 3 is large. Note that the expenditure shares on each commodity are equal for countries 2 and 3 .

To get a preliminary idea of the variation in multilateral shares that the above example generates, we first table $S^{2} / S^{1}$ and $S^{3} / S^{1}$ for the Paasche and Laspeyres star systems where the price vector for each country is used to value outputs. Thus in Table 1, Methods 1-3 correspond to the indexes $p^{1} \cdot y^{i} / p^{1} \cdot y^{1}, p^{2} \cdot y^{i} / p^{2} \cdot y^{1}, p^{3} \cdot y^{i} / p^{3} \cdot y^{1}$ for $i=2,3$.

TABLE 1: Paasche and Laspeyres Star and Fisher Star Systems.

\begin{tabular}{|l|c|c|c|c|c|c|c|}
\hline & Method 1 & Method 2 & Method 3 & Method 4 & Method 5 & Method 6 & Method 7 \\
\hline & $\begin{array}{c}\text { Country 1 } \\
\text { Prices }\end{array}$ & $\begin{array}{c}\text { Country 2 } \\
\text { Prices }\end{array}$ & $\begin{array}{c}\text { Country 3 } \\
\text { Prices }\end{array}$ & $\begin{array}{c}\text { Fisher } \\
\text { Star 1 }\end{array}$ & $\begin{array}{c}\text { Fisher } \\
\text { Star 2 }\end{array}$ & $\begin{array}{c}\text { Fisher } \\
\text { Star 3 }\end{array}$ & $\begin{array}{c}\text { Blended } \\
\text { Fisher }\end{array}$ \\
\hline$S^{2} / S^{1}$ & 33.67 & 1.96 & 49.76 & 8.12 & 8.12 & 5.79 & 7.25 \\
\hline$S^{3} / S^{1}$ & 336.67 & 930.49 & 9.95 & 57.88 & 81.25 & 57.88 & 64.12 \\
\hline
\end{tabular}

Examining Table 1, we see that using the prices of each country to value every country's quantity vector (Methods 1-3) causes the share of country 2 relative to $1, S^{2} / S^{1}$, to range from about 2 to 50 while $S^{3} / S^{1}$ ranges from about 10 to 980 . We also calculated the Fisher star relative shares in Table 1 (Methods 4-6); see equations (56) with $Q=Q_{F}$. We find that using the Fisher star systems, the relative share variation is dramatically reduced but still is quite big: $S^{2} / S^{1}$ ranges from about 5.8 to 8.1 while $S^{3} / S^{1}$ ranges from about 58 to 81 . We would expect that a satisfactory multilateral method should generate relative shares $S^{2} / S^{1}$ and $S^{3} / S^{1}$ that fall in the ranges spanned by the Fisher stars-namely 5.8 
to 8.1 and 58 to 81 respectively. The Fisher blended shares defined by (57) are listed as Method 7 in Table 1.

TABLE 2: Exchange Rate and Average Price and Quantity Methods.

\begin{tabular}{|l|c|c|c|c|c|}
\hline & $\begin{array}{c}\text { Eethod 8 } \\
\text { Rate }\end{array}$ & $\begin{array}{c}\text { Method 9 } \\
\text { Average } \\
\text { Quantities }\end{array}$ & $\begin{array}{c}\text { Method 10 } \\
\text { Average } \\
\text { Quantities }\end{array}$ & $\begin{array}{c}\text { Geary- } \\
\text { Khamis }\end{array}$ & $\begin{array}{c}\text { Van Yzeren } \\
\text { Average } \\
\text { Quantities }\end{array}$ \\
\hline$S^{2} / S^{1}$ & 6.67 & 7.33 & 1.49 & 47.39 & 1.32 \\
\hline$S^{3} / S^{1}$ & 66.67 & 60.87 & 11.86 & 57.35 & 13.24 \\
\hline
\end{tabular}

In Table 2, we listed the exchange rate and average price and average quantity methods that were defined in section 4-9 of the main text of this paper. Method 8 is the exchange rate method; see equations (1). This method does rather well in our artificial model, probably because the geometric mean of prices in each country is identical. Hence there are no grossly overvalued or undervalued country exchange rates. We would not expect this good performance to carry over to examples where some countries had grossly overvalued exchange rates.

Turning now to the average price methods defined in section 5 , we find that the arithmetic and geometric mean price methods defined by (15) and (16) generate equal average prices. Hence both of these methods are equivalent to Method 1 in Table 1 where the equal prices of country 1 were used to value quantities in each country.

Method 9 is the Walsh [1901; 431]-Fisher [1922; 307] arithmetic mean average quantity method defined by (22) and (23) while Method 10 the Walsh [1901; 398]-Gerardi [1982; 398] geometric mean average quantity method defined by (22) and (24). The arithmetic mean quantity vector turns out to be $[334,37.3]$ while the geometric mean quantity vector is $[10,12.6]$. Thus Methods 9 and 10 generate quite different relative shares in Table 2.

The Geary [1958]-Khamis [1970] average prices method defined in section 7 is Method 11. The vector of international prices turns out to be $[.05261, .47369]$, which is closest to the structure of relative prices in the large country, country 3 . This method seems to lead to a tremendous overevaluation of the share of country $2 ; S^{2} / S^{3}$ for the GK method is $47.39 / 57.35=.83$, which seems too large.

Van Yzeren's [1957; 13] unweighted average price method defined in section 8 is the next method we consider. The international price vector $\left[p_{1}^{*}, p_{2}^{*}\right]$ defined by (33) turns out to be $[1,1]$, so again, this method reduces to Method 1. Van Yzeren's [1957; 6-14] unweighted average quantity method defined in section 9 is Method 12. The vector of 
average quantities defined by (43) for this method turns out to be $\left[y_{1}^{*}, y_{2}^{*}\right] \equiv[.99342,1]$. This method leads to a share for country 1 which is too large.

Table 3 lists the superlative methods discussed in sections 10-13 with the bilateral $Q$ equal to $Q_{F}$, the Fisher ideal quantity index.

TABLE 3: Superlative Methods Using the Fisher Bilateral Index.

\begin{tabular}{|l|c|c|c|c|}
\hline & Method 13 & Method 14 & Method 15 & Method 16 \\
\hline & Gini-EKS & $\begin{array}{c}\text { Unweighted } \\
\text { Balanced }\end{array}$ & Own Share & $\begin{array}{c}\text { Weighted } \\
\text { Balanced }\end{array}$ \\
\hline$S^{2} / S^{1}$ & 7.2563 & 7.2563 & 6.024 & 6.005 \\
\hline$S^{3} / S^{1}$ & 64.8064 & 64.8063 & 59.970 & 59.697 \\
\hline
\end{tabular}

The effects of weighting are evident in Table 3. The two superlative methods that satisfy the country partitioning Test $T 10$ (Methods 15 and 16) have shares that are relatively close to the big country's Fisher star shares (Method 6) while the two superlative methods that do not satisfy $T 10$ (Methods 13 and 14) have shares that are very close to the arithmetic average of the Fisher star shares (Method 7, a democratically weighted method).

The above numerical example shows that the choice of a multilateral method is very important from an empirical point of view-more important than the choice of a bilateral index number formula in the time series context because the variation in relative prices and quantities will usually be much greater in the multilateral context. Even when choosing between superlative multilateral methods, we see that there can be substantial differences between Methods 13 and 14 (which pass the linear homogeneity test T8) and Methods 15 and 16 (which pass the country partitioning test $T 10$ ).

If the quantity vector for country 1 is changed to $y_{1} \equiv\left(y_{1}^{1}, y_{2}^{1}\right) \equiv(1,1)$, then the expenditure shares on each commodity will equal $1 / 2$ in each country. Hence for this new data set, the data are consistent with economic agents maximizing the utility function $f\left(y_{1}, y_{2}\right) \equiv y_{1}^{1 / 2} y_{2}^{1 / 2}$ subject to country budget constraints. This functional form is a special case of (41) and (65) (with $a_{11} \equiv a_{22} \equiv 0$ and $a_{12} \equiv 1 / 2$ ) and hence the Fisher and Walsh bilateral quantity indexes defined by (2) and (64) will empirically pass the circularity test (52). (The direct and indirect Persons [1928; 21-22] - Törnqvist [1936] quantity indexes $Q_{0}$ and $\tilde{Q}_{0}$ defined in Diewert $[1976 ; 120-121]$ will also pass the circularity test for this data set since Cobb-Douglas utility functions are exact for these functional forms as well). For this modified data set, the entries in Table 1 for the Fisher star methods, Methods 4-6, all reduce to $S^{2} / S^{1}=10$ and $S^{3} / S^{1}=100$. In this case, all of the superlative methods 
listed in Table 3 also have $S^{2} / S^{1}=10$ and $S^{3} / S^{1}=100$. Thus it is deviations from circularity of the bilateral index number formula that cause the superlative methods to yield different numerical results. As an aside, for this circular data set, it should be noted that the Geary-Khamis relative shares are $S^{2} / S^{1}=90.13$ and $S^{3} / S^{1}=108.73$. Thus the share of country 2 still seems to be too large in this case. To further illustrate that Geary-Khamis indexes can be quite different from Fisher ideal indexes, consider Table 4 where the Geary-Khamis bilateral index number formula (31) was used to form star system shares. The results using countries 1-3 respectively as the base country are tabled in columns 1-3 and are compared with the common Fisher star shares in column 4.

TABLE 4: Geary-Khamis Star Shares Versus Fisher Shares Using the Circular Data.

\begin{tabular}{|l|c|c|c|c|}
\hline & Geary-Khamis 1 & Geary-Khamis 2 & Geary-Khamis 3 & Fisher \\
\hline$S^{2} / S^{1}$ & 2.92 & 2.92 & 17.23 & 10 \\
\hline$S^{3} / S^{1}$ & 20.76 & 3.50 & 20.76 & 100 \\
\hline
\end{tabular}

We now return to our original noncircular data set and calculate the 4 superlative indexes when we use the bilateral Walsh quantity index $Q_{W}$ defined by (64) in place of the bilateral Fisher quantity index $Q_{F}$ defined by (2).

In Table 5, we list the Walsh star shares $S^{2} / S^{1}$ and $S^{3} / S^{1}$ using countries 1-3 as the base (see equations (56) which define the star shares) which are Methods 17-19. We also list the corresponding Fisher-Walsh blended shares defined by (57) where $Q=Q_{W}$ (Method 20).

TABLE 5: Walsh Star Shares and Walsh Blended Shares.

\begin{tabular}{|l|c|c|c|c|}
\hline & Method 17 & Method 18 & Method 19 & Method 20 \\
\hline & Walsh Star 1 & Walsh Star 2 & Walsh Star 3 & Blended Shares \\
\hline$S^{2} / S^{1}$ & 9.167 & 9.167 & 5.238 & 7.603 \\
\hline$S^{3} / S^{1}$ & 52.381 & 91.667 & 52.381 & 61.381 \\
\hline
\end{tabular}

Comparing Table 5 with Table 1, it can be seen that the Walsh star shares are less variable than the country price star shares (Methods 1-3), but the Walsh star shares (Methods 17-19) are more variable than the Fisher star shares (Methods 4-6). Thus the Walsh star relative shares, $S^{2} / S^{1}$, range from about 5.2 to 9.2 (while the corresponding 
Fisher variation was 5.8 to 8.1 ) and the Walsh relative shares, $S^{3} / S^{1}$, range from about 52 to 92 (while the corresponding Fisher variation was 58 to 81). Since the Fisher indexes satisfy circularity better than the Walsh indexes, we would expect that the variation in the 4 Walsh superlative indexes will be greater than the variation in the 4 Fisher superlative indexes. This expectation is verified by the results of Table 6 .

TABLE 6: Superlative Methods Using the Walsh Bilateral Index.

\begin{tabular}{|l|c|c|c|c|}
\hline & Method 21 & Method 22 & Method 23 & Method 24 \\
\hline & Gini-Walsh & $\begin{array}{c}\text { Unweighted } \\
\text { Balanced }\end{array}$ & Own Share & $\begin{array}{c}\text { Weighted } \\
\text { Balanced }\end{array}$ \\
\hline$S^{2} / S^{1}$ & 7.6067 & 7.6068 & 5.630 & 5.572 \\
\hline$S^{3} / S^{1}$ & 63.1227 & 63.1227 & 55.892 & 55.196 \\
\hline
\end{tabular}

Comparing Table 6 with Table 3, we see some similarities: the democratically weighted Gini-Walsh and Walsh unweighted balanced methods (Methods 21 and 22) closely approximate each other, while the plutocratically weighted Walsh own share and Walsh weighted balanced methods (Methods 23 and 24) also approximate each other reasonably closely. However, the spread between the methods that satisfy the linear homogeneity test $T 8$ (Methods 21 and 22) and the methods that satisfy the country partitioning test $T 10$ (Methods 23 and 24) is much wider in Table 6 then it was in Table 3 where the more nearly circular Fisher bilateral indexes were used as the basic building blocks. In Table 6, note that the shares corresponding to the plutocratic Methods 23 and 24 are closer to the shares of the big country star shares (Method 19) whereas the shares corresponding to the equally weighted Methods 21 and 22 are very close to the arithmetic average of the Walsh star shares (Method 20).

The fact that empirically the Fisher bilateral indexes satisfy the circularity test more closely than the Walsh indexes reinforces the case for preferring the Fisher index over its bilateral competitors. In addition to being superlative and satisfying more reasonable tests than its competitors, the Fisher ideal quantity index is the only superlative index that is consistent with (bilateral) revealed preference theory; see Diewert [1976; 137]. Thus we prefer the Fisher superlative methods listed in Table 3 over the Walsh superlative methods listed in Table 6.

We conclude this appendix by calculating the international prices that were suggested at the end of section 14 for our noncircular data set. 
TABLE 7: International Prices Using Own Share Price Levels and GK Prices.

\begin{tabular}{|l|c|c|c|}
\hline & Balk's Method & Hill's Method & Geary-Khamis \\
\hline$\pi_{2} / \pi_{1}$ & 8.895 & 9.027 & 9.004 \\
\hline
\end{tabular}

Balk's suggested vector of international prices $\pi \equiv\left(\pi_{1}, \pi_{2}\right)$ was defined by (86) and the generalized Hill prices were defined by (25) where the price levels (or purchasing power parities) $P^{k}$ and the country shares $S^{k}$ which appear in these equations were defined by the analyst's "best" multilateral method. In Table 7, we used the Fisher own share $P^{k}$ and $S^{k}$ (see Method 15 in Table 3) in equations (86) and (25) to calculate the Balk and Hill international prices. Both of these international price relatives $\pi_{2} / \pi_{1}$ are close to the Geary-Khamis international price relative, $\pi_{2} / \pi_{1}=9.004$. Recall that the structure of relative prices in the three countries is $p_{2}^{1} / p_{1}^{1}=1, p_{2}^{2} / p_{1}^{2}=.01$ and $p_{2}^{3} / p_{1}^{3}=100$ for countries 1-3 respectively. Thus the international price ratios in Table 7 all tend to lean towards the structure of relative prices in the big country, country 3 . Note that if we used the Balk or Hill international prices to value the quantity vectors in each country, the resulting country shares of world consumption at these constant prices would be very close to the Geary-Khamis shares (see Method 11 in Table 2) and these shares are very different from the shares generated by our suggested best methods listed in Table 3 .

Our numerical example suggests that additive multilateral methods should not be used if the structure of relative prices is very different across countries. In this case, no single international price vector can adequately represent the prices faced by producers or consumers in each country. In order to model adequately the very large substitution effects that are likely to be present in this situation, an economic approach based on the use of superlative indexes should be used. 


\section{Footnotes}

* Research Associate of the National Bureau of Economics Research and Professor of Economics at the University of British Columbia. This research was supported by a SSHRC grant. The author thanks Bert Balk and Alice Nakamura for helpful suggestions and Tina Corsi for typing a difficult manuscript.

1. See Diewert [1992] and Balk [1995a]. Balk [1995a; 87] argues that the Fisher and Sato [1976] - Vartia [1974] [1976] price indexes are both the best from the viewpoint of the test or axiomatic approach to index number theory. However, the Sato-Vartia price and quantity indexes are not superlative and hence are not "best" from the perspective of the economic approach. In addition, Reinsdorf and Dorfman [1995] have shown that the Sato-Vartia indexes do not satisfy the monotonicity axioms that the Fisher indexes satisfy.

2. The study of symmetric multilateral indexes dates back to Walsh [1901; 398-431], Fisher [1922; 297-308] and Gini [1924] [1931]. Early researchers who suggested desirable properties or tests for multilateral indexes include Drechsler [1973; 18-21], Gerardi [1982; 395-398] and Hill [1982; 50] [1984; 130-132].

3. See Diewert [1976].

4. The "countries" could be different regions or producer establishments. The list of commodities consumed (or produced) by the "countries" must be the same.

5. We interpret $y_{n}^{k}$ as the total amount of commodity $n$ consumed (or produced) in country $k$ during the relevant time period and $p_{n}^{k}$ as the corresponding average price or unit value. If commodity $n$ is not consumed (or produced) in country $k$ during the period under consideration, then $p_{n}^{k}>0$ is interpreted as the Hicksian $[1940 ; 114]$ reservation price that would just induce the consumer to purchase 0 units of good $n$ (or just induce the producer to supply 0 units of good $n$ ). This is the convention on the positivity of prices and quantities used by Armstrong [1995].

6. Notation: $y \geq 0_{N}\left(y>>0_{N}\right)$ means that each component of the $N$ dimensional column vector $y$ is nonnegative (strictly positive); $y>0_{N}$ means $y \geq 0_{N}$ but $y \neq 0_{N}$ and $p^{T} y=p \cdot y \equiv \Sigma_{n=1}^{N} p_{n} y_{n}$ denotes the inner product of the vectors $p$ and $y$. The transpose of the column vector $y$ is $y^{T}$.

7. See Walsh [1901] [1921], Fisher [1911] [1922], Eichhorn and Voeller [1976], Diewert [1992; 214-223] [1993a; 33-34] and Balk [1995a]. 
8. In the producer context, we assume either: (i) each producer in country $k$ minimizes input cost $p^{k} \cdot y$ subject to a production function constraint $f(y)=f\left(y^{k}\right)$ where $f$ is increasing, linearly homogeneous and concave, or (ii) each producer in country $k$ maximizes revenue $p^{k} \cdot y$ subject to the constraint $f(y)=f\left(y^{k}\right)$ where $f$ is an increasing, linearly homogeneous and convex factor requirements function. In case (ii), $c(p)$ defined by (6) is to be interpreted as a unit revenue function; see Diewert [1974][1976; 125].

9. See Diewert [1993b; 117].

10. The aggregator function $f$ is restricted to be linearly homogeneous, strictly increasing $\left(\nabla f(y)>>0_{N}\right.$ for $\left.y>0_{N}\right)$ and concave in the consumer context and in the cost minimizing producer context but convex in the revenue maximizing producer context.

11. The unit cost function $c$ is restricted to be linearly homogeneous, weakly increasing $\left(\nabla c(p)>0_{N}\right.$ for $\left.p>>0_{N}\right)$ and concave in the consumer context and in the cost minimizing producer context but convex in the revenue maximizing producer context.

12. See Diewert [1976; 117 and 134].

13. We follow Diewert [1993c; 361] and define a homogeneous symmetric mean $m\left(x_{1}\right.$, $\left.\ldots, x_{N}\right)$ to be a continuous, symmetric, increasing and positively linearly homogeneous function which has the mean value property $m(\lambda, \lambda, \ldots, \lambda)=\lambda$.

14. The terms price and quantity levels are due to Eichhorn $[1978 ; 141]$.

15. Geary [1958] defined the method and Khamis [1970] [1972] showed that the defining equations have a positive solution.

16. More accessible references are Debreu and Herstein [1953; 598] and Karlin [1959; 246-256].

17. Geary $[1958 ; 98]$ first exhibited this formula for the case $K=2$.

18. For additional tests, see Martini [1992] and Balk [1995a].

19. Given $s \equiv\left[S^{1}, \ldots, S^{K}\right]^{T}$ and $\alpha \equiv 1 / \lambda$, the vector of international prices $p^{*}$ can be defined as $p^{*} \equiv \alpha \Sigma_{k=1}^{K}\left(p^{k} \cdot y^{k}\right)^{-1} p^{k} S^{k}$. It should be noted that the $d_{i j}$ are Afriat's [1967] cross-coefficients.

20. See Diewert [1976; 116 and 133-134] for proofs and references to the literature.

21. Frisch $[1930 ; 399]$ called (51) the product test. The concept of the test is due to Fisher [1911; 418]. 
22. The concept of the test is due to the statistician Westergaard [1890;218-219].

23. We assume that $Q$ satisfies the identity test: $Q\left(p^{i}, p^{j}, y, y\right)=1$. In sections $10-13$, we denote the share and price level of country $k$ by $S_{k}$ and $P_{k}$ respectively instead of using our previous notation $S^{k}$ and $P^{k}$. This is done because reciprocals $S_{k}^{-1}$ and powers $S_{k}^{2}$ of the $S_{k}$ will appear in the defining equations for these methods.

24. This is what Kravis $[1984 ; 10]$ calls the "star system" with country 1 as the star.

25. Fisher [1922; 274-276] was writing about price indexes but his arguments apply also to quantity indexes.

26. We require only BT1 and BT3 to get the constant price weights representation (A36) in the Appendix.

27. The Cobb-Douglas price weights bilateral quantity index defined in Proposition 7 fails the crucial bilateral test BT4.

28. Fisher $[1922 ; 305]$ actually averaged price indexes (using each time period as the base) rather than quantity indexes.

29. Gini [1924] [1931] was concerned only with making multilateral price comparisons but his analysis can be adapted to the quantity comparison situation as we have indicated.

30. If $Q$ does not satisfy the time reversal test, then use the Walsh [1921] rectification procedure and obtain the solution ray to (61) by replacing $Q\left(p^{j}, p^{k}, y^{j}, y^{k}\right)$ in (63) by $Q^{*}\left(p^{j}, p^{k}, y^{j}, y^{k}\right) \equiv\left[Q\left(p^{j}, p^{k}, y^{j}, y^{k}\right) / Q\left(p^{k}, p^{j}, y^{k}, y^{j}\right)\right]^{1 / 2}$.

31. The solution ray defined by (63) does indeed solve (61) since the objective function is bounded from below by 0 , unbounded from above and there is only one ray of critical points.

32. It should be noted that the equality between (60) and (61) is due to Van Ijzeren [1987; 62-63] except that he restricted himself to the use of Fisher, Paasche and Laspeyres price or quantity indexes.

33. For historical references to the originators of the corresponding tests for price indexes, see Diewert $[1992 ; 214-221]$. For the bilateral tests, we assume that $p^{1}>>$ $0_{N}, p^{2}>0_{N}, y^{1}>>0_{N}$ and $y^{2}>>0_{N}$.

34. See Diewert $[1992 ; 221]$. 
35. This unit cost function was originally defined in Diewert [1971] where it was shown to be a flexible functional form. It is the special case of the quadratic mean of order $r$ unit cost function that occurs when $r=1$; see Diewert [1976; 130].

36. See Diewert $[1986 ; 28][1988 ; 69]$.

37. See also Van Ijzeren [1983; 44] [1987; 60-61] and Balk [1989], who provided an excellent exposition of the balanced method and derived some new properties for it.

38. Gerardi [1974] let $P\left(p^{j}, p^{k}, y^{j}, y^{k}\right)=p^{k} \cdot y^{k} / p^{j} \cdot y^{k}$, the Paasche price index. Van ljzeren $[1987 ; 61]$ later let $P$ be the Laspeyres, Paasche and Fisher price indexes.

39. Note that if we sum equations (70) over $i$, we get an identity and hence any one of the equations (70) can be dropped. A normalization on the $P_{k}$ will make a positive solution to (70) unique.

40. If this procedure does not converge, then use Van Yzeren's [1956; 17] slightly more complicated procedure. Van Yzeren $[1956 ; 27-29]$ proves convergence of this latter iterative scheme.

41. If $Q$ empirically satisfies circularity, then the base invariant shares $S_{1}^{*}, \ldots, S_{K}^{*}$ will also satisfy equations (58) and (67); i.e., the Gini system, the own share system, the unweighted balanced system and the weighted balanced system to be studied in the next section all collapse down to the same system of shares.

42. Van Ijzeren [1987; 63-64] made a different theoretical argument showing why the three variants of the unweighted balanced method will be numerically close.

43. Van Ijzeren $[1983 ; 45-46]$ chose the bilateral quantity index $Q$ to be either the Laspeyres quantity index $Q_{L}$, the Paasche quantity index $Q_{P}$ or the Fisher ideal quantity index $Q_{F}$.

44. Our method of proof is an adaptation of Van ljzeren's [1987; 65] and Balk's [1995; 8] method of proof.

45. Note that it is much easier to calculate the weighted balanced shares with a general $Q$ then it is to calculate the unweighted balanced shares where a closed form solution does not seem to exist.

46. Other notable multilateral methods that we have not studied due to limitations of space and time include methods due to Iklé [1972] (see also Dikhanov [1994]), Van Ijzeren 
[1983; 45] [1987; 64-67] Diewert [1986][1988], Kurabayashi and Sukuma [1990], Hill [1995] and Balk [1995b].

47. This equivalent performance of the own share and the weighted balanced method was also obtained by Balk $[1989 ; 310]$ for his set of axioms.

48. This close numerical approximation property is verified for our numerical example described in Appendix 2.

49. This theoretical approximation result is verified for our numerical example described in Appendix 2.

50. If $K=2$, Proposition 5 shows that Van Yzeren's unweighted average price method is a superlative additive system. Another example of a superlative additive method when $K=2$ is the Walsh-Gerardi system defined by (3) and (16). In this case, $S^{2} / S^{1}=$ $Q_{W}\left(p^{1}, p^{2}, y^{1}, y^{2}\right)$ where $Q_{W}$ is the Walsh $[1901 ; 552]$ quantity index defined by $(64)$ above.

51. For statements of this effect, see Gini [1931; 14], Drechsler [1973; 26], Gerardi [1982; 383], P. Hill [1982; 54] [1984; 128], Kravis [1984; 8-9], Marris [1984; 52] and R.J. Hill [1995; ch. 4]. In the producer theory context, the indifference curve through $A, B, C$ is replaced with a production possibilities curve which has the opposite curvature. Hence the biases are reversed in the producer theory context.

52. Gini $[1931 ; 14]$ had a clear understanding of substitution bias in the context of consumer price indexes.

53. For further references to the use of unit values to aggregate commodities over time and place, see Diewert $[1995 ; 28]$ and Balk [1995c].

54. Of course, the resulting constant international "dollar" country aggregate values $\pi \cdot y^{k}$ will not generally be proportional to the country shares $S^{k}$ generated by the "best" multilateral method.

55. Actually, only tests $T 2, T 3, T 9, T 10$ and $T 11$ are new and some of these tests are straightforward modifications of existing tests.

56. However, since the present author introduced this method, the reader should be aware of a potential bias problem in this recommendation. 


\section{REFERENCES}

Afriat, S.N. [1967], "The Construction of Utility Functions from Expenditure Data", International Economic Review 8, 67-77.

Armstrong, K.G. [1995], Multilateral Approaches to the Theory of International Comparisons, Ph.D. Thesis, University of British Columbia, Vancouver, Canada.

Balk, B.M. [1989], "On Van Ijzeren's Approach to International Comparisons and Its Properties", Statistical Papers (Statistische Hefte) 30, 295-315.

Balk, B.M. [1995a], "Axiomatic Price Index Theory: A Survey", International Statistical Review 63, 69-93.

Balk, B.M. [1995b], "A Comparison of Ten Methods For Multilateral International Price and Volume Comparisons", Department of Statistical Methods, Statistics Netherlands, P.O. Box 4000, $2270 \mathrm{JM}$ Voorburg, July, forthcoming in the Journal of Official Statistics.

Balk, B.M. [1995c], "On the Use of Unit Value Indices as Consumer Price Subindices", March 14, Division of Research and Development, Statistics Netherlands, P.O. Box 959, 2270 AZ Voorburg, The Netherlands.

Debreu, G. and I.N. Herstein [1953], "Nonnegative Square Matrices", Econometrica 21, 597-607.

Diewert, W.E. [1971], "An Application of the Shephard Duality Theorem: A Generalized Leontief Production Function", Journal of Political Economy, 79, 481-507.

Diewert, W.E. [1974], "Functional Forms for Revenue and Factor Requirements Functions", International Economic Review 15, 119-130.

Diewert, W.E. [1976], "Exact and Superlative Index Numbers", Journal of Econometrics $4,115-145$.

Diewert, W.E. [1981], "The Economic Theory of Index Numbers: A Survey", pp. 163-208 in Essays in the Theory and Measurement of Consumer Behavior in Honour of Sir Richard Stone, A. Deaton (ed.), London: Cambridge University Press.

Diewert, W.E. [1986], "Microeconomic Approaches to the Theory of International Comparisons", Technical Working Paper No. 53, National Bureau of Economic Research, Cambridge, MASS.

Diewert, W.E. [1988], "Test Approaches to International Comparisons", pp. 67-86 in Measurement in Economics, W. Eichhorn (ed.), Heidelberg: Physica-Verlag. 
Diewert, W.E. [1992], "Fisher Ideal Output, Input and Productivity Indexes Revisited", The Journal of Productivity Analysis 3, 211-248.

Diewert, W.E. [1993a], "The Early History of Price Index Research", pp. 33-65 in Essays in Index Number Theory, Volume 1, W.E. Diewert and A.O. Nakamura (eds.), Amsterdam: North-Holland.

Diewert, W.E. [1993b], "Duality Approaches to Microeconomic Theory", pp. 105-175 in Essays in Index Number Theory, Volume 1, W.E. Diewert and A.O. Nakamura (eds.), Amsterdam: North-Holland.

Diewert, W.E. [1993c], "Symmetric Means and Choice Under Uncertainty", pp. 355-433 in Essays in Index Number Theory, Volume 1, W.E. Diewert and A.O. Nakamura (eds.), Amsterdam: North-Holland.

Diewert, W.E. [1996], "Price and Volume Measures in the System of National Accounts", pp. 237-285 in The New System of National Accounts, J.W. Kendrick (ed.), Boston: Kluwer Academic Publishers.

Dikhanov, Y. [1994], "Sensitivity of PPP Based Income Estimates to Choice of Aggregation Procedures", paper presented at the Conference of the International Association for Research in Income and Wealth, St. Andrews, New Brunswick, August.

Drechsler, [1973], "Weighting of Index Numbers in Multilateral International Comparisons", The Review of Income and Wealth, Series 19 (March), 17-47.

Eichhorn, W. [1976], "Fisher's Tests Revisited", Econometrica 44, 247-256.

Eichhorn, W. [1978], Functional Equations in Economics, London: Addison-Wesley.

Eichhorn, W. and J. Voeller [1976], Theory of the Price Index, Berlin: Springer-Verlag.

Eltetö, O. and P. Köves [1964], "On a Problem of Index Number Computation Relating to International Comparisons", Statisztikai Szemle 42, 507-518.

Fisher, I. [1911], The Purchasing Power of Money, London: Macmillan.

Fisher, I. [1922], The Making of Index Numbers, Boston: Houghton Mifflen.

Frisch, R. [1930], "Necessary and Sufficient Conditions Regarding the Form of an Index Number which shall Meet Certain of Fisher's Tests", American Statistical Association Journal 25, 397-406.

Frobenius, G. [1908], "Über Matrizen aus positiven Elementen", Sitzungsberichte der königlich preussischen Akademie der Wissenschaften Part 1, 471-476. 
Frobenius, G. [1909], "Über Matrizen aus positiven Elementen II", Sitzungsberichte der königlich preussischen Akademie der Wissenschaften Part 1, 514-518.

Gale, D. and H. Nikaido [1965], "The Jacobian Matrix and the Global Univalence of Mappings", Mathematische Annalen 159, 81-93.

Geary, R.G. [1958], "A Note On Comparisons of Exchange Rates and Purchasing Power Between Countries", Journal of the Royal Statistical Society A, 121, 97-99.

Gerardi, D. [1974], "Sul problema della comparazione dei poteri d'acquisto delle valute", Serie Papers, Instituto di Statistica dell'Universita di Padova, August.

Gerardi, D. [1982], "Selected Problems of Inter-Country Comparisons on the Basis of the Experience of the EEC", Review of Income and Wealth, Series 28, 381-405.

Gini, C. [1924], "Quelques considérations au suject de la construction des nombres indices des prix et des questions analogues", Metron 4:1, 3-162.

Gini, C. [1931], "On the Circular Test of Index Numbers", Metron 9:2, 3-24.

Hicks, J.R. [1940], "The Valuation of the Social Income", Economica 7, 105-140.

Hicks, J.R. [1946], Value and Capital, Second Edition, Oxford: Clarendon Press.

Hill, R.J. [1995], Purchasing Power Parity Methods of Making International Comparisons, Ph.D. thesis, University of British Columbia, Vancouver, Canada.

Hill, P. [1982], Multilateral Measurements of Purchasing Power and Real GDP, Luxembourg: Eurostat.

Hill, T.P. [1984], "Introduction: The Special Conference on Purchasing Power Parities", Review of Income and Wealth, Series 30, 125-133.

Iklé, D.M. [1972], "A New Approach to the Index Number Problem, The Quarterly Journal of Economics 86, 188-211.

Jevons, W.S. [1884], Investigations in Currency and Finance, London: Macmillan.

Karlin, S. [1959], Mathematical Methods and Theory in Games, Programming and Economics, Volume 1, Reading MA.: Addison-Wesley.

Khamis, S.H. [1970], "Properties and Conditions for the Existence of a New Type of Index Number", Sankhya Series B 32, 81-98.

Khamis, S.H. [1972], "A New System of Index Numbers for National and International Purposes", Journal of the Royal Statistical Society, A, 135, 96-121. 
Kravis, I.B. [1984], "Comparative Studies of National Incomes and Prices", Journal of Economic Literature 22, 1-39.

Kravis, I.B., Z. Kenessey, A. Heston, and R. Summers [1975], A System of International Comparisons of Gross Product and Purchasing Power, Baltimore: The Johns Hopkins University Press.

Kravis, I.B., R. Summers and A. Heston [1982], "Comments on 'Selected Problems of Intercountry Comparisons on the Basis of the Experience of the EEC"', Review of Income and Wealth, Series 28, 407-410.

Kurabayashi, Y. and I. Sakuma [1990], Studies in International Comparisons of Real Product and Prices, Economic Research Series No. 28, The Institute of Economic Research, Hitotsubashi University, Tokyo: Kinokuniya Co.

Leontief, W. [1936], "Composite Commodities and the Problem of Index Numbers", Econometrica 4, 39-59.

Marris, R. [1984], "Comparing the Incomes of Nations: A Critique of the International Comparison Project", Journal of Economic Literature 22:1, 40-57.

Martini, M. [1992], "A General Function of Axiomatic Index Numbers", Journal of the Italian Statistical Society 3, 359-376.

Perron, O. [1907], "Grundlagen für eine Theorie des Jacobischen Kettenbruchalgorithmus", Mathematische Annalen 64, 1-76.

Persons, W.M. [1928], The Construction of Index Numbers, Cambridge, Massachusetts: The Riverside Press.

Pierson, N.G. [1896], "Further Considerations on Index-Numbers", Economic Journal 6, $127-131$

Reinsdorf, M.B. and A.H. Dorfman [1995], "The Sato-Vartia Index and the Monotonicity Axiom", Washington, D.C.: US Bureau of Labor Statistics.

Rudin, W. [1953], Principles of Mathematical Analysis, New York: McGraw-Hill Book Co.

Sato, K. [1976], "The Ideal Log-Change Index Number", The Review of Economics and Statistics 58, 223-228.

Shephard, R.W. [1953], Cost and Production Functions, Princeton, N.J.: Princeton University Press.

Szulc, B. [1964], "Indices for Multiregional Comparisons", Przeglad Statystyczny (Statistical Review), 3, 239-254. 
Törnqvist, L. [1936], "The Bank of Finland's Consumption Price Index", Bank of Finland Monthly Bulletin 10, 1-8.

Van Yzeren, J. [1957], Three Methods of Comparing the Purchasing Power of Currencies, Statistical Studies No. 7, The Hague: The Netherlands Central Bureau of Statistics.

Van Ijzeren, J. [1983], Index Numbers for Binary and Multilateral Comparison, Statistical Studies No. 34, The Hague: The Netherlands Central Bureau of Statistics.

Van Ijzeren, J. [1987], Bias In International Index Numbers: A Mathematical Elucidation, Eindhoven: Dissertatiedrukkerij Wibro.

Vartia, Y.O. [1974], Relative Changes and Economic Indices, Licenciate thesis, Department of Statistics, University of Helsinki.

Vartia, Y.O. [1976], "Ideal Log-Change Index Numbers", Scandanavian Journal of Statistics 3, 121-126.

Vogt, A. [1980], "Der Zeit und der Faktorumkehrtests als 'Finders of Tests'", Statistiche Hefte 21, 66-71.

Walsh, C.M. [1901], The Measurement of General Exchange Value, New York: Macmillan and Co.

Walsh, C.M. [1921], "Discussion of the Best Form of Index Number", Journal of the American Statistical Association 17, 537-544.

Westergaard, H. [1890], Die Grundzüge der Theorie der Statistik, Jena.

Wold, H. [1944], "A Synthesis of Pure Demand Analysis, Part III", Skandinavisk Aktuarietidskrift 27, 69-120. 